\title{
Home visiting program for older persons with poor health status
}

Citation for published version (APA):

Bouman, A. I. E. (2008). Home visiting program for older persons with poor health status. [Doctoral Thesis, Maastricht University]. Datawyse / Universitaire Pers Maastricht. https://doi.org/10.26481/dis.20080611ab

Document status and date:

Published: 01/01/2008

DOI:

10.26481/dis.20080611ab

Document Version:

Publisher's PDF, also known as Version of record

\section{Please check the document version of this publication:}

- A submitted manuscript is the version of the article upon submission and before peer-review. There can be important differences between the submitted version and the official published version of record.

People interested in the research are advised to contact the author for the final version of the publication, or visit the DOI to the publisher's website.

- The final author version and the galley proof are versions of the publication after peer review.

- The final published version features the final layout of the paper including the volume, issue and page numbers.

Link to publication

\footnotetext{
General rights rights.

- You may freely distribute the URL identifying the publication in the public portal. please follow below link for the End User Agreement:

www.umlib.nl/taverne-license

Take down policy

If you believe that this document breaches copyright please contact us at:

repository@maastrichtuniversity.nl

providing details and we will investigate your claim.
}

Copyright and moral rights for the publications made accessible in the public portal are retained by the authors and/or other copyright owners and it is a condition of accessing publications that users recognise and abide by the legal requirements associated with these

- Users may download and print one copy of any publication from the public portal for the purpose of private study or research.

- You may not further distribute the material or use it for any profit-making activity or commercial gain

If the publication is distributed under the terms of Article $25 \mathrm{fa}$ of the Dutch Copyright Act, indicated by the "Taverne" license above, 
Home visiting program for older persons with poor health status 
Home visiting program for older persons with poor health status

ISBN 978-90-5278-718-3

Universitaire Pers Maastricht

Lay-out Yvonne Jaegers and Ans Bouman

Cover design Frie Hoekstra

Production Datawyse

(C) A.I.E. Bouman, Amsterdam, 2008

All rights reserved. No part of this thesis may be reproduced or transmitted in any form or by any means, electronic or mechanical, including photocopying, recording or any information storage or retrieval system, without permission in writing from the author, or, when appropriate, from the publishers of the publications. 


\title{
Home visiting program for older persons with poor health status
}

\author{
PROEFSCHRIFT \\ ter verkrijging van de graad van doctor \\ aan de Universiteit Maastricht, \\ op gezag van de Rector Magnificus, \\ Prof.mr. G.P.M.F. Mols, \\ volgens het besluit van het College van Decanen, \\ in het openbaar te verdedigen \\ op woensdag 11 juni 2008 om 14.00 uur \\ door \\ ANNEKE IDA EMILIE BOUMAN
}

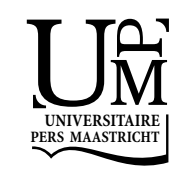


Promotores

Prof.dr. G.I.J.M. Kempen

Prof.dr. P.G. Knipschild

Copromotor

Dr. H.J.L. van Rossum

Beoordelingscommissie

Prof.dr. J.P.H. Hamers (voorzitter)

Prof.dr. G. Kok

Prof.dr. M.G.M. Olde Rikkert (UMC St. Radboud Nijmegen)

Prof.dr. M.H. Prins

Prof.dr. A.E. Stuck (University of Bern, Switzerland)

This project was sponsored by Health Grant 22000115 from the Netherlands Organization for Health Research and Development (ZonMw), The Hague, the Netherlands.

The trial is registered with Current Controlled Trials (CCT) through the International Standard Randomised Controlled Trial Number Register [ISRCTN92017183].

The studies presented in this thesis were conducted at the Department of Epidemiology (Maastricht University), and were embedded in the School for Public Health and Primary Care (CAPHRI). CAPHRI participates in the Netherlands School of Primary Care Research (CaRe). CaRe was accredited by the Royal Netherlands Academy of Arts and Sciences (KNAW). 
Op welke manieren kunnen we die leeftijdsfase verdragen waarin alles zwaarder wordt?

Cicero (106-43 v.Chr.)

De kunst van het oud worden

aan Alex, Ilse, en mijn ouders 



\section{Contents}

Chapter 1 General introduction 9

Chapter 2 Effects of home visits by home nurses to elderly people $\quad 15$ with health problems: design of a randomized clinical trial in the Netherlands

Chapter 3 Home visiting program for older people with health problems: process evaluation

Chapter 4 Effects of a home visiting program for older people with poor health status: a randomized clinical trial in the Netherlands

Chapter 5 Effects on health care use and associated cost of a home visiting program for older people with poor health status: a randomized clinical trial in the Netherlands

Chapter 6 Effects of intensive home visiting programs for older people with poor health status: a systematic review

Chapter 7 General discussion

Appendix 1 De gezondheidstoestand van zelfstandig wonende ouderen: resultaten van een schriftelijke enquête in Sittard en Geleen (The health status of community-living elderly: results of a postal questionnaire in the south of the Netherlands)

Summary

Samenvatting (Summary in Dutch)

Dankwoord (Acknowledgements in Dutch)

About the author 

1

General introduction 


\section{Introduction}

The number of older persons aged 65 years and over is increasing. Europe currently has the highest proportion of older persons and this is projected to remain so for at least the next 50 years. About $30 \%$ of the European population is estimated to be 65 or over in 2050, up from $8 \%$ in 1950 and $15 \%$ in 2000 . The Netherlands is not a front-runner in Europe; it is considered to be a relatively 'young' country. In 2050, the percentage of older persons in the Netherlands is forecasted to be $26 \%$; only for Denmark the percentage is lower $(25 \%)$, while for Spain (37\%) and Greece (34\%) the percentages are strikingly higher. ${ }^{1}$ The life expectancy for persons born in the Netherlands in 2050 is also projected to increase, to 82 years for men and 84 years for women; this is up from 71 years for men and 73 years for women in 1950, and from 76 years and 81 years, respectively, in $2000 .^{2}$ With the growth in the ageing population, the prevalence of disease and disability increases. Not surprisingly, this leads to higher care utilization, and a large proportion of health care expenditures in the Netherlands is now related to older persons. ${ }^{3}$

In 1964, Williamson and colleagues found that many of the multiple health problems and disabilities present in older persons living at home in Scotland were not known to their general practitioner. ${ }^{45}$ They suggested that 'health visitors could undertake periodic visiting of old people and carry out screening' and that 'medical and social measures, applied soon enough' would prevent functional impairment. In the decades to follow, preventive programs were developed, and one of such programs is home visitation by public health nurses of older persons living in the community. Key elements of these (systematic) visits mostly are: assessment of health problems and risks, advice, and referral to professional or community services. The aim of the visits is to maintain or improve the functional abilities and well-being of older persons and subsequently reduce the use of (more expensive) institutional care services. Such programs are part of national policy in several countries, including Denmark, Japan and Australia. However, there is still an ongoing debate whether home visits should be incorporated into regular care for older persons as the findings of trials on the effectiveness of home visits have been inconsistent. ${ }^{6}$

A trial in the Netherlands in the early 1990s showed that preventive home visits do not seem to be useful for the general population of older persons. ${ }^{78}$ In that trial, experienced public health nurses visited the older persons in the intervention group at least four times a year over a period of 3 years; older persons in the control group received usual care. After 3 years, no or hardly any effects were demonstrated on health outcomes and service use of the total group of visited older persons, although a subgroup analysis revealed that the visits seemed to have been effective for older persons with a poor perceived health status. Visited persons with poor health at baseline $(n=57)$ scored considerably 
better on several health measures, for example on functional status, compared to similar persons in the control group $(\mathrm{n}=53)$; mortality rates after 3 years were lower in the intervention group and beneficial effects were found for hospital admissions, especially re-admissions. These effects already emerged during the first year of the intervention. The results of this trial indicated that preventive home visits may be effective when restricted to those with a poor perceived health status. With the exception of a Danish ${ }^{9}$ and an Italian trial ${ }^{10}$ which reported (partly) positive results, other studies reported small $^{11}{ }^{12}$ or no effects ${ }^{13} 14$ in the general population of older persons. The probable usefulness of home visits in a risk-group approach has been confirmed in four controlled studies, ${ }^{15-18}$ but the results of three other trials do not support this

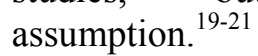

Although home visits for a high-risk population seems to be a promising approach, further evidence is needed; the findings of the Dutch trial mentioned were based on relatively small numbers of participants, whereas other studies rendered inconsistent results. We therefore carried out a new study in the Netherlands in which the risk-group approach is not only tested in a larger population of those with perceived poor health, but in which we also test whether nurses with a less-intensive training (enrolled home nurses instead of public health nurses) are able to obtain convincing effects. This is the subject of the thesis.

\section{Objectives}

The main objective of this thesis is to assess the effects of systematic home visits by home nurses to older persons with perceived health problems in terms of their health status, use of care services and cost-effectiveness. Beforehand we hypothesized that the visits will improve the functional abilities, perceived health and quality of life of the older persons. We also hypothesized that these will reduce institutionalization, especially hospital (re-) admissions, and total health care expenditures.

\section{Outline of the thesis}

The study is conducted as a randomized, clinical trial in cooperation with a large home care organization in the south of the Netherlands (Sittard and surrounding areas). A detailed design of the trial is presented in chapter 2. The process evaluation of the home visiting program is provided in chapter 3 . The purpose of this evaluation is to give insight into factors which may play a role in the effectiveness of the program, such as the content of the home visits and the adherence to the given recommendations. The experiences of the participants and the nurses with the program are also discussed. In chapter 4 we report on the effectiveness of the program on health-related measures, e.g. self-rated health, functional status and quality of life. These were measured at regular intervals using mailed questionnaires and through individual interviews. The 
effects on health care use and associated cost, including a cost-effectiveness analysis, are described in chapter 5. The volumes of the professional health services and goods, which were used by the participants during the intervention period and a 6-month follow-up period, were mostly obtained from computerized databases of various medical administration offices. In chapter 6 , a systematic literature review on the effects of intensive home visiting programs is presented in which we integrate our results with evidence from comparable studies targeting older persons in poor health. Finally, in chapter 7, the major findings of the trial are reflected in the general discussion. The Appendix (a report in Dutch) describes the results of a postal survey on the health status of community-living older persons (aged 70-84, $\mathrm{n}=4,901$ ) in the south of the Netherlands. The survey also served as screening instrument for the participants in the trial and provided baseline information.

Chapters 2-6 have been published in international journals. The chapters can be read independently from each other; inevitably there is an overlap with respect to the background and the design of the study. Small differences in wording or lay-out may exist between the journal publications and the corresponding articles presented in this thesis. 


\section{References}

1. Population Division, Department of Economic and Social Affairs, United Nations [http://www.unpopulation.org].

2. CBS [http://www.statline.cbs.nl].

3. Meerding WJ, Bonneux L, Polder JJ, Koopmanschap MA, van der Maas PJ. Demographic and epidemiological determinants of healthcare costs in the Netherlands: cost of illness study. BMJ 1998;317(7151):111-5.

4. Williamson J, Stokoe IH, Gray S, Fisher M, Smith A, McGhee A, et al. Old People at Home. Their Unreported Needs. Lancet 1964;13:1117-20.

5. Stuck AE, Beck JC, Egger M. Preventing disability in elderly people. Lancet 2004;364(9446):1641-2.

6. van Haastregt JC, Diederiks JP, van Rossum E, de Witte LP, Crebolder HF. Effects of preventive home visits to elderly people living in the community: systematic review. BMJ 2000;320(7237):754-8.

7. van Rossum E. Effects of preventive home visits to the elderly. PhD Thesis Maastricht University, 1993.

8. van Rossum E, Frederiks CM, Philipsen H, Portengen K, Wiskerke J, Knipschild P. Effects of preventive home visits to elderly people. BMJ 1993;307(6895):27-32.

9. Hendriksen C, Lund E, Stromgard E. Consequences of assessment and intervention among elderly people: a three year randomised controlled trial. BMJ Clin res ed 1984;289(6457):1522-4.

10. Bernabei R, Landi F, Gambassi G, Sgadari A, Zuccala G, Mor V, et al. Randomised trial of impact of model of integrated care and case management for older people living in the community. BMJ Clin res ed 1998;316(7141):1348-51.

11. Fabacher D, Josephson K, Pietruszka F, Linderborn K, Morley JE, Rubenstein LZ. An inhome preventive assessment program for independent older adults: a randomized controlled trial. J Am Geriatr Soc 1994;42(6):630-8.

12. Wagner EH, LaCroix AZ, Grothaus L, Leveille SG, Hecht JA, Artz K, et al. Preventing disability and falls in older adults: a population-based randomized trial. Am J Publ Health 1994;84(11):1800-6.

13. McEwan RT, Davison N, Forster DP, Pearson P, Stirling E. Screening elderly people in primary care: a randomized controlled trial.[comment]. Brit J Gen Pract 1990;40(332):94-7.

14. Vetter NJ, Lewis PA, Ford D. Can health visitors prevent fractures in elderly people? BMJ Clin res ed 1992;304(6831):888-90.

15. Hall N. Randomized trial of a health promotion program for frail elders. Canadian Journal on Aging/La Revue Canadienne du vieillissement 1992;11(1):72-91.

16. Pathy MS, Bayer A, Harding K, Dibble A. Randomised trial of case finding and surveillance of elderly people at home. Lancet 1992;340(8824):890-3.

17. Leveille SG, Wagner EH, Davis C, Grothaus L, Wallace J, LoGerfo M, et al. Preventing disability and managing chronic illness in frail older adults: a randomized trial of a community-based partnership with primary care.[comment]. J Am Geriatr Soc 1998;46(10):1191-8.

18. Robichaud L, Hébert R, Roy PM, Roy C. A preventive program for community-dwelling elderly at risk of functional decline: a pilot study. Arch Gerontol and Geriatr 2000;30:73-84.

19. Stuck AE, Minder CE, Peter Wuest I, Gillmann G, Egli C, Kesselring A, et al. A randomized trial of in-home visits for disability prevention in community-dwelling older people at low and high risk for nursing home admission. Archives of Internal Medicine 2000;160(7):97786.

20. Dalby DM, Sellors JW, Fraser FD, Fraser C, van Ineveld C, Howard M. Effect of preventive home visits by a nurse on the outcomes of frail elderly people in the community: a randomized controlled trial. Canadian Medical Association Journal / Journal de l'Association Medicale Canadienne 2000;162(4):497-500. 
Chapter 1

21. van Haastregt JC, Diederiks JP, van Rossum E, de Witte LP, Voorhoeve PM, Crebolder HF. Effects of a programme of multifactorial home visits on falls and mobility impairments in elderly people at risk: randomised controlled trial.[comment]. BMJ 2000;321(7267):994-8. 


\section{2}

\section{Effects of home visits by home nurses to elderly people with health problems: design of a randomized, clinical trial in the Netherlands}

Ans Bouman ${ }^{1}$, Erik van Rossum ${ }^{1}$, Gertrudis Kempen ${ }^{2}$ and Paul Knipschild ${ }^{3}$

BMC Health Serv Res 2004, 4(1):35

1 Department of Epidemiology, Maastricht University, Maastricht, the Netherlands

2 Department of Health Care Studies, Maastricht University, Maastricht, the Netherlands

3 Department of General Practice, Maastricht University, Maastricht, the Netherlands 


\begin{abstract}
Background

Preventive home visits to elderly people by public health nurses aim to maintain or improve the functional status of elderly and reduce the use of institutional care services. A number of trials that investigated the effects of home visits show positive results, but others do not. The outcomes can depend on differences in characteristics of the intervention program, but also on the selection of the target population. A risk group approach seems promising, but further evidence is needed. We decided to carry out a study to investigate the effects in a population of elderly with (perceived) poor health rather than the general population. Also, we test whether nurses who are qualified at a lower professional level (home nurses instead of public health nurses) are able to obtain convincing effects. The results of this study will contribute to the discussion on effective public health strategies for the aged.
\end{abstract}

\title{
Methods/design
}

The study is conducted as a parallel-group randomized trial. To screen eligible participants, we sent a postal questionnaire to 4,901 elderly people aged 70-84 living at home in a town in the south of the Netherlands. After applying inclusion criteria (e.g. self-reported poor health status) and exclusion criteria (e.g. those who already receive home nursing care), we selected 330 participants. They entered the randomization procedure; 160 were allocated to the intervention group and 170 to the control group. The intervention consists of (at least) eight systematic home visits over an 18-month period. Experienced home nurses from the local home care organization carry out the visits. The control group receives usual care. Effects on health status are measured by means of postal questionnaires after 12,18 (the end of the intervention period) and after 24 months (the end of a 6-month follow-up period), and individual interviews after 18 months. Data on mortality and service use are continuously registered during 24 months. A cost-benefit analysis is included.

The design and setting of the study, the selection of eligible participants and the study interventions are described in this article. Other included items are: the primary and secondary outcome measures, the statistical analysis and the economic evaluation. 


\section{Background}

The number of elderly people is increasing. Due to the ageing population, more demands are made on health care services. ${ }^{1}$ In the last two decades, preventive programs have been developed aiming at reducing health care cost and improving the independent functioning of elderly people. One of such programs is home visitation by public health nurses of elderly people living in the community. This aims to maintain or improve the functional abilities and wellbeing of elderly people and reduce the use of institutional care services. Such programs for elderly people are part of national policy in several countries, including the United Kingdom, Denmark and Australia. However, the results of trials on the effects of home visits have been inconsistent. ${ }^{2}$ Investigators are still in search of the most effective strategy.

In the past years three reviews were published on the effects of preventive home visits to elderly people living in the community. ${ }^{2-4}$ These used different methodological approaches. Apart from a number of similar trials, each review also included a series of different trials, depending on the inclusion criteria and the date of publication. Van Haastregt et al. reviewed 15 studies and concluded that no clear evidence exists for the effectiveness of the visits: the observed effects are considered to be fairly modest and inconsistent. ${ }^{2}$ Nine trials reported at least one (significant) favorable effect and six trials reported no effects. In most of the studies the intervention was aimed at the general population aged 65 years or over, without any selection. The other two reviews included a metaanalysis of the data and were more positive about the effects of home visits. ${ }^{34}$ Stuck et al. indicated that home visits can reduce the risk of functional decline and nursing home admission, provided that the interventions are based on a multidimensional geriatric assessment and include multiple follow-up visits. ${ }^{4}$ Home visiting programs improved functional status more in people with the lowest mortality risk (younger population, $<80$ years). Elkan et al. reported a favorable effect on mortality and nursing home admissions among members of the general population and frail older people who are at risk of adverse outcomes. ${ }^{3}$ However, they did not find improvement in functional status.

One can argue about the differences in the approach of each review, but in general the results of the home visiting studies are heterogeneous with respect to the different outcome measures. Many factors can play a role in the effectiveness of the interventions, including the target population, characteristics of the intervention, the persons carrying out the visits and the compliance with the given advice.

Research in the Netherlands showed that preventive home visits do not seem to be useful for the general population of elderly people. ${ }^{5}$ In that trial, experienced public health nurses visited the intervention group $(n=300)$ at least four times a year over a period of 3 years. The control group $(n=300)$ received usual care. After 3 years, no or hardly any effects were demonstrated on the health care and service use of the total group of visited elderly people (see Table 
1). However, a subgroup analysis indicated that the visits seemed to be effective for elderly people with poor (perceived) health status. Visited persons with poor health at baseline scored considerably better on several health measures (e.g. functional status) compared to similar persons in the control group. Mortality rates after 3 years were lower (24\% versus $40 \%$ ) and substantial effects were found for referrals to outpatient clinics (61\% versus $79 \%)$ and also for hospital admissions, especially re-admissions. In the intervention subgroup $47 \%$ were admitted at least once to the hospital, with a total of 1,134 days; in the control subgroup these figures were $74 \%$ and 2,043 days (Table 1). These effects emerged already during the first year of the intervention period. ${ }^{6}$

Table 1 Mortality and use of services (percentages) for all participants and for those who rated their health status as poor at the start of the study

\begin{tabular}{|c|c|c|c|c|c|}
\hline \multirow{3}{*}{$\begin{array}{l}\text { Results of study by van } \\
\text { Rossum et al. }\end{array}$} & \multicolumn{2}{|l|}{ Total population } & \multicolumn{3}{|c|}{$\begin{array}{l}\text { Population with poor perceived } \\
\text { health }\end{array}$} \\
\hline & $\begin{array}{l}\text { Intervention } \\
\text { group }\end{array}$ & $\begin{array}{l}\text { Control } \\
\text { group }\end{array}$ & $\begin{array}{l}\text { Intervention } \\
\text { group }\end{array}$ & & $\begin{array}{l}\text { ontrol } \\
\text { group }\end{array}$ \\
\hline & $(n=292)$ & $(n=288)$ & $(n=57)$ & & $n=53)$ \\
\hline Mortality & $41 \quad(14 \%)$ & $49(17 \%)$ & 14 (24\%) & 21 & $(40 \%)$ \\
\hline $\begin{array}{l}\text { Referrals to outpatient } \\
\text { clinics }\end{array}$ & $132(55 \%)$ & $166(66 \%)$ & 27 (61\%) & 38 & $(79 \%)$ \\
\hline $\begin{array}{l}\text { Hospital } \\
\text { (re-) admissions }\end{array}$ & $121(41 \%)$ & $133(46 \%)$ & $27 \quad(47 \%)$ & 39 & $(74 \%)$ \\
\hline number of days & 3,838 & 4,789 & 1,134 & & 2,043 \\
\hline $\begin{array}{l}\text { Admission to home for } \\
\text { older persons }\end{array}$ & $20(7 \%)$ & $18(6 \%)$ & $12(21 \%)$ & 7 & $(13 \%)$ \\
\hline
\end{tabular}

The probable usefulness of home visits for a high-risk group was confirmed in five controlled studies. ${ }^{7-11}$ However, the results of three other trials did not support this assumption. ${ }^{12-14}$ Although home visits for a restricted population seems a promising approach, further evidence is needed. The findings of the earlier Dutch subgroup analysis were based on a relatively small number of subjects (53 control and 57 intervention). Therefore, we decided to carry out a new trial in which the risk-group approach is tested in a larger population of those with (perceived) poor health. At the same time, we appointed nurses who are qualified at a lower professional level (enrolled home nurses instead of public health nurses) to carry out the visits. An experienced public health nurse will supervise them.

This study will investigate the effects of systematic home visits by home nurses to elderly people with (perceived) health problems in terms of their health status, the use of care services and the cost-effectiveness. We expect that 
the visits will improve the functional abilities, perceived health and quality of life of the participants. We also hypothesize that they will reduce specialist care, institutionalization, especially hospital (re-) admissions, and total health care expenditures. Evidence regarding the usefulness of the proposed risk-group approach is needed to decide on the future implementation of the visits. This article presents the design of this new trial.

\section{Methods/design}

\section{Study design and setting}

The study is conducted as a parallel-group randomized trial. It is conducted in cooperation with a large home care organization in the south of the Netherlands (Sittard and surrounding areas). The addresses we used to screen eligible persons for the study were drawn from the population register of the municipality. After the screening procedure we randomized 330 elderly people. Effects of the intervention are measured by means of postal questionnaires after 12,18 (at the end of the intervention period) and after 24 months (at the end of a 6-month follow-up period) and by individual interviews after 18 months. Mortality and data on the use of care services are continuously registered over the 24-month research period. A cost-benefit analysis is also included. The design of the study is shown in Figure 1. The design is, unless otherwise mentioned, implemented according to plan.

The study has obtained the approval by the Medical Ethical Committee of Maastricht University/Academic Hospital Maastricht.

\section{Identification of eligible participants}

We sent a postal questionnaire to 4,901 elderly people between the age of 70 and 84 years who were still living at home. These lived in 14 districts in the research area. We included districts with close proximity to the centre of town where the home care organization is situated. In this way we limited the travelling time of the nurses to carry out the visits. Districts with large industrial areas were excluded.

Reminders were sent after $2-3$ weeks to $45 \%$ of the elderly people. The response rate was $76 \%$ after about 6 weeks. The response rates per district fluctuated between $65 \%$ and $81 \%$. The average time to fill out the questionnaire was about 30 minutes. The elderly people could do this by themselves or, if they needed help, with the assistance of family, friends or volunteers. A list of names and addresses of volunteers was added to the questionnaire. Even if persons did not want to participate in the study, we kindly requested them to fill out the questionnaire and return it to us. A postage free envelope was included.

The questionnaire was used as a screening instrument and also provided baseline measurements for the participants of the trial. Among the respondents 
( $n=3,689$, see Figure 1), we found 872 persons who reported their health status as poor (on a scale ranging from 1-10 points: scores 1-5 are considered poor, 67 fairly good, and 8-10 good). Our previous home visitation study indicated positive effects for this subgroup. Five persons did not sign the informed consent form and 273 persons with poor health status did not want to participate in the study. Of the remaining 594 persons, we excluded those who already received home nursing care at baseline, in order to avoid contamination of (other) nursing care. Referral to nursing services after the start of the intervention period has no consequences for the scheduled home visits in the intervention group. It is regarded as a possible effect of the intervention and it is registered as outcome in terms of service use. Persons on a waiting list for admission to nursing homes or homes for older persons were also excluded. The local independent committee dealing with applications for the use of care services already granted them this service. It is likely that most of them already receive regular supervision of professional caregivers. Six persons were excluded on the advice of their general practitioner (GP). They were severely or terminally ill and would probably die within 6 months. On the basis of these exclusion criteria, a total of 102 persons were excluded.

After applying the in- and exclusion criteria, 492 persons were eligible to take part in the study. However, we excluded 162 more persons for the following reasons: their GP did not want to cooperate with the study $(n=139)$, respondents had too many missing values on the functional status scale $(n=11)$, the health insurance company was unknown $(n=1)$ or it was uncertain whether the health insurance company would be willing to cooperate $(n=11)$. The health insurance companies of $96 \%$ of the finally selected participants had already given consent to provide us with data on health service use. Because we selected persons who's GP was willing to cooperate, relevant health care data from the GP practices are available for all participants. A flow diagram of the selection of participants is shown in Figure 1. Finally, 330 persons entered the randomization procedure. In consideration of the available working hours of the nurses, the maximum number of participants to receive home visits was 160 . The control group was hence set at 170 .

\section{Sample size consideration}

We calculated the sample size from the data of our previous home visitation study in the Netherlands. ${ }^{5}$ Participants were categorized on the primary outcome measure self-rated health, perceiving their health status as (a) better or the same compared to the start of the study, or (b) worse or deceased. We expect to demonstrate a difference of $20 \%$ between the study groups $(65 \%$ score (a) in group I versus $45 \%$ in group II). Based on a 0.9 power to detect a significant difference $(\alpha=0.05$, one-sided), 104 participants are required for both study groups. 


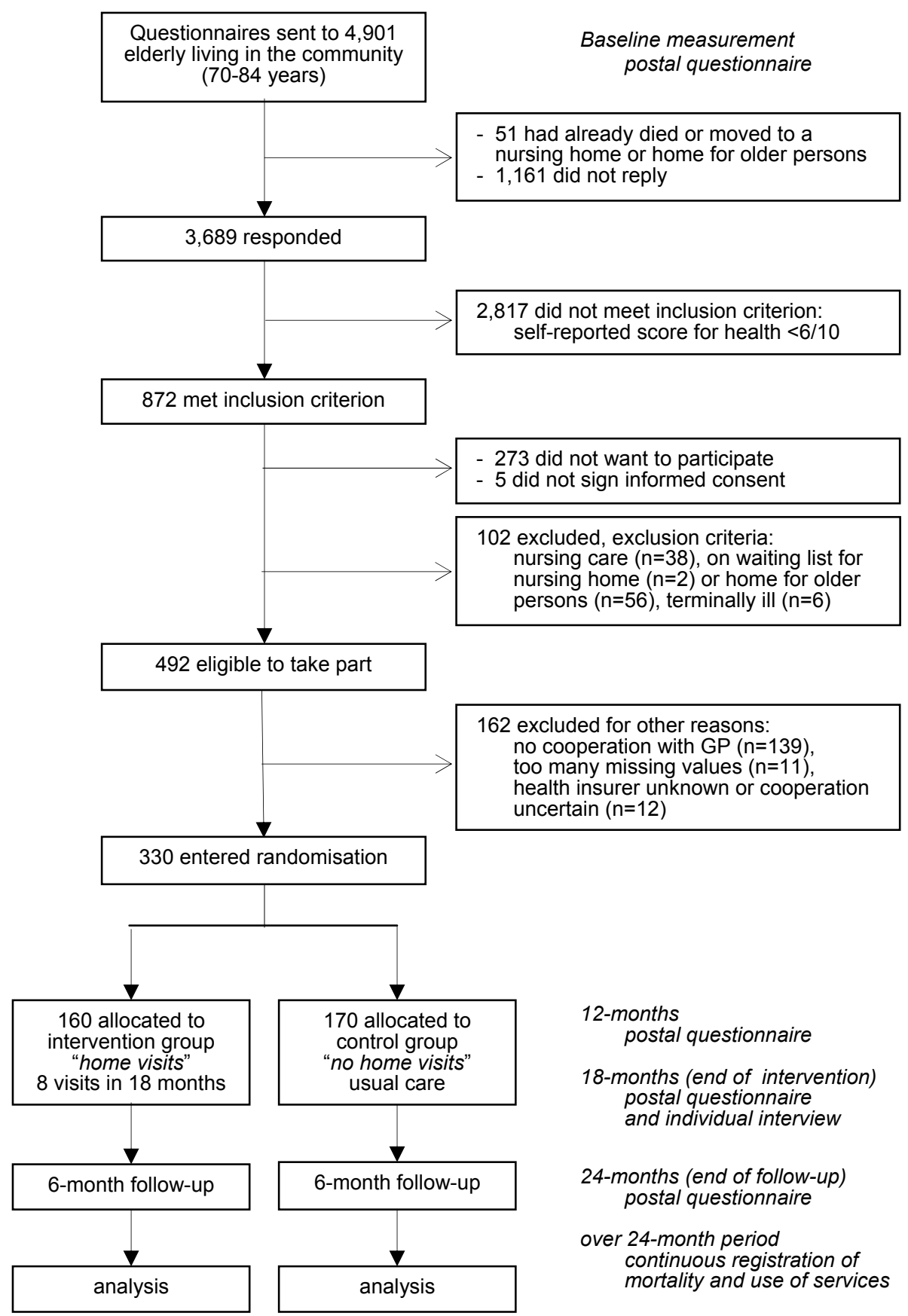

Figure 1 Study design (The summary of the trial design includes: the screening procedure, the randomization, the intervention and the points in time at which the effects of the intervention are measured.) 
Accounting for a loss to follow up of 30\%, we planned to enrol 150 participants per group. This number is also large enough (again extrapolated from our own data) to detect differences in specialist care and institutionalization (e.g. to detect a difference in mean hospital days of 10 days over a 1.5 year period).

Based on data of the selection criteria we estimated that about $10 \%$ of the screened population was eligible for the study (including informed consent). Therefore, we needed to mail a minimum of 3,500 questionnaires to persons aged 70-84 years living in the community. To account for unforeseen circumstances, we decided to send out about 5,000 questionnaires. After applying the inclusion and exclusion criteria, and taking into account the GPs' willingness to cooperate with the study, there were sufficient participants eligible for the study to raise the selected number to 330 . This slightly increased the power of the study.

\section{Randomization}

The baseline measurements included questions on relevant prognostic factors related to the health status and service use. Before randomization, we divided the 330 participants into two groups: couples $(n=46)$ and those for whom this did not apply $(\mathrm{n}=284)$. In this way we made sure that eligible persons who lived together were always allocated to the same study group (in order to avoid contamination of the intervention). The 23 couples were divided into three strata on the basis of their (added) score on functional status (0-4, 5-7 or more than 7 out of 18 activities that cannot be performed independently). The other 284 participants were stratified into eight strata based on three prognostic factors two health-variables and one service use-variable:

1. functional status (0-2 or more than two activities that the elderly people cannot perform independently)

2. changes in health during the 3 months prior to completion of the questionnaire (same/better or worse)

3. contact with a medical specialist in the 3 months prior to completion of the questionnaire (contact yes or no).

The participants in each of the eight strata were then randomized into either a control or intervention group using a computer generated randomization list with a block length of four. ${ }^{15}$ Randomly, we allocated 160 persons to the intervention group and 170 persons to the control group. Table 2 shows the baseline characteristics of the intervention and control group. The study groups are well matched; there were hardly any differences between the groups at the start of the study.

The participants in the intervention group were assigned to one of the three home nurses. This depended on the location of their GP practice, because each nurse was assigned to a number of GP practices. We assumed that this would facilitate the cooperation with the GPs. Each nurse was responsible for 52-56 older persons during the intervention period. 


\section{Study interventions}

Our previous study showed that positive effects of the visits for people with a poor health status emerged already within 1.5 years. In the new trial the intervention period is restricted to this period. At the same time we increased the frequency of the visits. This enables the nurses to intervene more promptly on identified problems and risks, and to establish a position of trust in a shorter time period. Experienced home nurses therefore visit the intervention group at least eight times over an 18-month period. If necessary, extra visits can be made. The duration of the visits can last between 60 and 90 minutes. Participants in the control group receive usual care; they can use or apply for all available services in the area.

Three nurses work half time for the trial. An experienced public health nurse supervises the visits on a weekly basis. All three home nurses are recruited from the cooperating home care organization. Home nurses, as well as public health nurses, are well trained to conduct home visits. They are embedded in community care organizations that traditionally have preventive tasks. The home nurses are not part of a multidisciplinary team, but advice can be obtained from in-home specialists within the home care organization, e.g. a dietician, a diabetes specialist and an occupational therapist. A nurse geriatric specialist from the local hospital can also be consulted, if necessary. At regular intervals, once every 6-8 weeks during the intervention period, he also advises the nurses on important geriatric issues.

\section{Home visit protocol}

The home visits can be described as 'systematic home visits to elderly people with health problems conducted by a home nurse'. The three most important elements of the visits are (1) to detect problems or risks, (2) to give advice and (3) to refer to other professional or community services. This brief description is applicable to all home visiting studies that have been carried out so far. However, there are large differences in the protocols that have been used in earlier studies, ranging from an interview to collect information on social and health conditions ${ }^{16}$ to a 'multidimensional geriatric assessment' in which medical, functional, psychosocial, and environmental evaluation of the problems and resources are assessed. ${ }^{12}{ }^{17}$ Earlier studies did not show any clear relation between the structure of the visits and the effects. So far, the active components of the intervention are not known yet, but a number of elements seem to be of importance for the content of the visits. We tried as much as possible to include these elements into the protocol: e.g. face-to-face assessment, good communication between the nurse and the elderly people including an empathic attitude by the nurse, an individual plan, a client-centred approach, good compliance with the given advice and multiple visits. ${ }^{418}$ 
Table 2 Baseline characteristics of the study participants

\begin{tabular}{|c|c|c|c|c|c|}
\hline \multirow{2}{*}{\multicolumn{2}{|c|}{$\begin{array}{l}\text { Characteristic } \\
\text { Age, mean } \pm \text { standard deviation }\end{array}$}} & \multicolumn{2}{|c|}{$\begin{array}{l}\text { Intervention group } \\
\qquad(n=160)\end{array}$} & \multicolumn{2}{|c|}{$\begin{array}{l}\text { Control group } \\
\quad(n=170)\end{array}$} \\
\hline & & 75.8 & (3.7) & 75.6 & (3.9) \\
\hline $\begin{array}{l}\text { Sex, } \mathrm{n}(\%) \\
\text { male } \\
\text { female }\end{array}$ & & $\begin{array}{l}64 \\
96\end{array}$ & $\begin{array}{l}(40) \\
(60)\end{array}$ & $\begin{array}{r}68 \\
102\end{array}$ & $\begin{array}{l}(40) \\
(60)\end{array}$ \\
\hline $\begin{array}{l}\text { Household composition } \\
\text { alone } \\
\text { together }\end{array}$ & (\%) & $\begin{array}{r}53 \\
103\end{array}$ & $\begin{array}{l}(34) \\
(66)\end{array}$ & $\begin{array}{r}61 \\
108\end{array}$ & $\begin{array}{l}(36) \\
(64)\end{array}$ \\
\hline $\begin{array}{l}\text { Education, } \mathrm{n}(\%) \\
\text { primary school } \\
\text { lower to middle profe } \\
\text { higher professional } €\end{array}$ & $\begin{array}{l}\text { sional education } \\
\text { ucation }\end{array}$ & $\begin{array}{l}60 \\
81 \\
15\end{array}$ & $\begin{array}{l}(39) \\
(52) \\
(10)\end{array}$ & $\begin{array}{l}65 \\
92 \\
11\end{array}$ & $\begin{array}{l}(39) \\
(55) \\
(6)\end{array}$ \\
\hline $\begin{array}{l}\text { Self-rated health*, n }(\% \\
\quad 1-4 \\
5\end{array}$ & & $\begin{array}{l}62 \\
98\end{array}$ & $\begin{array}{l}(39) \\
(61)\end{array}$ & $\begin{array}{r}67 \\
103\end{array}$ & $\begin{array}{l}(39) \\
(61)\end{array}$ \\
\hline $\begin{array}{l}\text { Functional status }{ }^{\dagger}, \mathrm{n} \text { (\% } \\
\text { ADL-dependencies } \\
\text { IADL-dependencies } \\
\text { Total number }\end{array}$ & $\begin{array}{l}0 \\
1-11 \\
0-1 \\
2-7 \\
0-2 \\
3-18\end{array}$ & $\begin{array}{l}73 \\
86 \\
76 \\
79 \\
83 \\
75\end{array}$ & $\begin{array}{l}(46) \\
(54) \\
(49) \\
(51) \\
(53) \\
(47)\end{array}$ & $\begin{array}{l}81 \\
89 \\
83 \\
82 \\
92 \\
76\end{array}$ & $\begin{array}{l}(48) \\
(52) \\
(50) \\
(50) \\
(55) \\
(45)\end{array}$ \\
\hline $\begin{array}{l}\text { Health change in previc } \\
\text { same or better } \\
\text { worse }\end{array}$ & s 3 months, n (\%) & $\begin{array}{l}85 \\
75\end{array}$ & $\begin{array}{l}(53) \\
(47)\end{array}$ & $\begin{array}{l}82 \\
88\end{array}$ & $\begin{array}{l}(48) \\
(52)\end{array}$ \\
\hline $\begin{array}{l}\text { Health affects social pa } \\
\text { often } \\
\text { sometimes } \\
\text { never }\end{array}$ & cipation, n (\%) & $\begin{array}{l}82 \\
49 \\
24\end{array}$ & $\begin{array}{l}(53) \\
(32) \\
(16)\end{array}$ & $\begin{array}{l}87 \\
50 \\
33\end{array}$ & $\begin{array}{l}(51) \\
(29) \\
(19)\end{array}$ \\
\hline $\begin{array}{l}\text { Contacted general pra } \\
3 \text { months, } n(\%)\end{array}$ & ioner in previous & 140 & (88) & 150 & $(88)$ \\
\hline $\begin{array}{l}\text { Contacted medical sp } \\
3 \text { months, } \mathrm{n}(\%)\end{array}$ & alist in previous & 108 & (69) & 117 & $(70)$ \\
\hline $\begin{array}{l}\text { Hospital admission in } \\
\mathrm{n}(\%)\end{array}$ & vious 3 months, & 21 & (13) & 24 & $(14)$ \\
\hline Use of home care, $\mathrm{n}(\%$ & & 64 & $(40)$ & 61 & (37) \\
\hline
\end{tabular}

Note: ADL or IADL-dependencies indicate the number of activities for which the older persons were dependant on others to perform the activity.

* Indicated by a score on a scale ranging from 1-10 points. Participants with poor health status (score $<6$ ) were included.

$\dagger$ Refers to 11 activities of daily living (ADLs) and seven instrumental (household) activities of daily living (IADLs). 
The visits are conducted in a systematic way according to a nursing model that distinguishes four steps: diagnosis, planning of activities, carrying out the activities and evaluation. ${ }^{19}$

\section{Diagnosis}

Our starting-point is a client-centred approach. The elderly people can indicate which problems they experience and which needs they have. The EasyCare Questionnaire, an elderly assessment system, is used to detect further problems. ${ }^{20}{ }^{21}$ Also, additional checklists are used on a variety of topics: e.g. vision, hearing and use of medication. A number of instruments are used for further diagnostic assessment: the get-up-and-go test, ${ }^{22}$ the Geriatric Depression Scale $^{23}$ and the Mini Mental State Examination. ${ }^{24}$ During the visits no physical examination takes place, because the home nurses are not qualified to do so. If necessary, the elderly people are referred to their GPs.

\section{Planning of activities}

An individual plan for each participant is set up. The activities are planned in agreement with the elderly people, as this will improve compliance. We only included older persons with a poor (perceived) health; hence a broad range of problems can come forward, including physical, mental as well as social problems. Guidelines on a number of geriatric topics are used for advice and referral regarding problems and risks that are identified. A Handbook of Nursing Diagnosis is also used to set up goals and interventions..$^{25} \mathrm{~A}$ maximum of three problems (and two interventions per problem) is being dealt with at one visit. Among the planned activities are referrals to professional or community services, and advice or information is given regarding, e.g. nutrition, social and physical activities and home aids.

\section{Carrying out the activities}

The older persons are primarily themselves responsible to carry out the planned activities. The home nurse only supports the elderly people. In order to improve compliance, the nurses contact the elderly people by telephone 1-4 weeks after each visit, depending on the type of advice. They ask whether the advice has been followed, and if not, what the impediments are and if further assistance is necessary. The participants are offered consultation with the nurses by telephone each morning between $9.00-9.30$ hours.

\section{Evaluation}

The evaluation of each home visit takes place at the next visit. The cycle is then repeated and new or old, but not solved, problems can be dealt with.

In the 3-month period before the start of the visits, the home nurses were actively involved in the development of the visiting protocol. They also received relevant training in communication skills and using assessment tools. They took courses on several subjects, e.g. relevant geriatric health topics, behaviour change and the usage of the Handbook of Nursing Diagnosis. ${ }^{25}$ 
Several pilot visits were conducted in which different aspects of the protocol were trained, e.g. using assessment tools and measuring instruments.

Communication between the nurses and the GPs is according to the 'normal' communication lines between nurses of the home care organizations and the GPs. Before the start of the study all GPs received a list of eligible participants registered at their practice to screen very ill persons. After randomization a definite list of participants was sent to them, but no reference was made to which treatment group they belong. The allocation of the participants to the two groups was disclosed after conclusion of the first three home visits. The GPs then received an overview of all treated problems for each participant in the intervention group, including the accompanying recommendations and the results of the interventions. The GPs were asked for their comments or suggestions and in this way they could become involved, if they wanted to. A similar overview will be sent to them for visits 4-6 and 7-8.

\section{Process evaluation}

All elements of the intervention are monitored as part of a process evaluation. This includes the registration of topics discussed at each visit, treated problems, advice given and referral to other services. The evaluation of each visit is registered at each next visit and includes the compliance with the given advice. Reasons for noncompliance are noted. The nurses' experiences with the visiting protocol, the role of the supervising public health nurse and the patient's experiences with the home visits will be assessed at the end of the intervention period by means of individual interviews.

Other aspects of the intervention process assessed are: the time spent on the visits, including the travelling and preparation time and the time spent on telephone contacts. Elements of the telephone conversation after each visit, most importantly whether the elderly people complied with the given advice, are registered.

Detailed analyses of the intervention process and outcome data may help to identify which program characteristics are related to possible favorable effects of the visits and may result in the development of more effective interventions. It might also provide additional information for the possible implementation of the visits in daily practice.

\section{Outcome measures}

The primary health related outcome measures are: self-rated health, functional status, quality of life and changes in self-reported problems. In addition, a variety of other health measures (secondary outcome measures) will be assessed. Information will be obtained, among other things, on health complaints, medication use, and loneliness and mental health. The municipality will supply mortality data (secondary outcome measure) over the entire research period. The use of services relates to the frequency and duration of care from 
the following services: domestic and community nursing care, GP, physiotherapy, day care in institutional care settings, hospital outpatient clinics, hospital, nursing home, home for older persons, use of aids and in-home modifications. The primary outcomes for service use are specialist medical care and hospital (re-) admission. The health insurance companies will supply data on the use of services over the 2-year research period. Additional data not covered by the health insurance companies will be supplied by, e.g. GPs, the hospital, and the home care organizations. Table 3 shows an overview of the outcome measures, their operationalization and at which time points the measures are carried out.

\section{Statistical analyses}

The main analyses will be conducted according to the intention-to-treat principle. Analysis of primary and secondary endpoints will be performed using relevant significance tests (e.g. chi-square, t-test or analysis of variance). Regression techniques will be used, if necessary, to estimate the effects for the various outcome measures, adjusting for small differences between the groups at the start of the study. In addition, we will conduct per-protocol analyses; these are restricted to those participants who complied fully with the intervention protocol and outcome measurements. Preplanned subgroup analysis will be performed for the following subgroups: living alone/together; health deterioration over the previous 3 months; functional status and locus of control. Differences in approaches between the nurses will be investigated.

\section{Economic evaluation}

A cost-effectiveness analysis will be conducted in which we consider cost from a societal perspective. The economic evaluation will measure and evaluate the 'real' cost. In this study we will include direct health care cost, i.e. cost made for the home visiting program and health care cost made by the participants. The cost of the intervention program consist of cost for the screening procedure, salaries of the nurses, travel expenses, cost of training sessions for the nurses, etc.

Health care cost include cost of inpatient and outpatient treatment, consultation by GPs and other medical practising specialists, physiotherapy, medication, professional home care, nursing home, meals on wheels, aids and appliances, etc. In order to estimate the cost, the quantity of each resource will be multiplied by its assigned unit cost of price. Direct non health care cost (e.g. the travel cost made by participants) is not included. These should preferably be gathered prospectively by means of a cost-diary. ${ }^{26}$ We considered this too burdensome for the participants. 
Chapter 2

Table 3 Outcome measures and their operationalization

\begin{tabular}{|c|c|c|}
\hline Outcome measure & Operationalization ${ }^{*}$ & Measurement $^{\dagger}$ \\
\hline Self-rated health ${ }^{\ddagger}$ & report mark, score 1-10 & $0,1,2,3$ \\
\hline Functional status $^{\ddagger}$ & $\begin{array}{l}\text { GARS },{ }^{28} \text { score } \underline{18}-72 \\
\text { ADL, subscore } \underline{11-44} \\
\text { IADL, subscore } \underline{7}-28\end{array}$ & $0,1,2,3$ \\
\hline Quality of life ${ }^{\ddagger}$ & $\begin{array}{l}\text { RAND- } 36,,^{29} 1 \text { item } \\
\text { SF-20, }{ }^{30} 2 \text { subscales, score } \\
0-\underline{100}\end{array}$ & $0,1,2,3$ \\
\hline $\begin{array}{l}\text { Changes in self-reported } \\
\text { problems }^{\ddagger}\end{array}$ & 3 main problems, 3-point scale & $0,1,2,3$ \\
\hline Health complaints & SCL- $90,{ }^{31} 2$ subscales & 4 \\
\hline Depressive complaints & GDS, ${ }^{23}$ score $\underline{0}-15$ & 4 \\
\hline Mental status & MMSE- $12,{ }^{32}$ score $0-\underline{12}$ & 4 \\
\hline Locus of control & Mastery Scale, ${ }^{33}$ score $7-\underline{35}$ & 4 \\
\hline Social support & SSL12-I, ${ }^{34}$ score $12-\underline{48}$ & 4 \\
\hline Loneliness & Loneliness Scale, ${ }^{35}$ score $\underline{0}-11$ & 4 \\
\hline Medication & volume, cost & 4 \\
\hline Aids and in-home modifications & type, cost & 4,5 \\
\hline Mortality & number & 5 \\
\hline $\begin{array}{l}\text { Use of extramural and institutional } \\
\text { care, e.g. medical specialist help }{ }^{\ddagger} \\
\text { and hospital (re-) admission }{ }^{\ddagger}\end{array}$ & $\begin{array}{l}\text { e.g. number of contacts GPs, } \\
\text { days in hospital, cost }\end{array}$ & 5 \\
\hline
\end{tabular}

* Underlined score indicates the most-favorable score on the specific scale.

$\dagger 0=$ postal questionnaire at the start of the study

$1=$ postal questionnaire after 12 months

2 = postal questionnaire after 18 months (at the end of the intervention period)

3 = postal questionnaire after 24 months (at the end of a 6 -month follow-up period)

$4=$ individual interview after 18 months

5 = continuous registration by services over a 24-month period.

$\ddagger$ Primary outcome measures.

Indirect health care cost (cost which are made during extra gained years of life) and indirect nonhealth care cost (the value of production lost to society due to illness-related absence from work and days of inactivity) are often also included in an economic evaluation. We decided however not to include those cost, because of their limited relevance in a population of retired elderly people. 


\section{Time plan for this study}

The screening procedure was conducted in the fall of 2002. In January 2003 we sent a letter to the older persons notifying them that they were selected to participate in the study and whether they would receive home visits or not. In February the home visits started. They are implemented according to plan and will end in September 2004. The first effect evaluation, 12 months after the start of the intervention period, has taken place: 302 questionnaires were sent out in March 2004. The response rate was 95\%. Since the beginning of the intervention period, a total of 24 participants died and four persons withdrew from the study. Three more effect evaluations will take place: two evaluations after the intervention period in October 2004 (postal questionnaire and individual interview) and one after the 6-month follow-up in March 2005 (postal questionnaire).

\section{Discussion}

The use of postal questionnaires turned out to be a good and inexpensive method to screen elderly people - there were more than sufficient eligible persons to participate in the research project. The response rate was high and less than one percent of the questionnaires were omitted due to too many missing values. For most of the variables, the percentage of missing values varied between 0 and 2 per cent. Media coverage shortly before sending out the questionnaires and accompanying letters from the municipality and the university may have contributed to the high response rate. It is not certain whether the results are comparable to other (larger) towns in the Netherlands. The response rate of the postal questionnaires used for the first effect evaluation (12 months after the start of the study) was 95\%. Nearly all included elderly people seemed to be motivated to participate.

We selected older persons with a poor perceived health status, because we expected the home visits to be more beneficial for this group rather than for those who are still in good health. ${ }^{5}$ Results from the data analysis of the first postal questionnaire (screening instrument and baseline measurement) showed that the eligible persons indeed scored worse on most health related variables, including functional status, mental health and social functioning. ${ }^{27}$

We considered including a third group of elderly people with poor health to receive home visits from voluntary workers. This was, however, not feasible, mainly because the number of participants with a participating GP was too low. The frequency of the visits and the level of professionalism, nurses versus voluntary aids (usually without any professional qualifications), could be a topic of study in another trial depending on the outcome of this study. 


\section{Acknowledgements}

The home care organization 'Thuiszorg Westelijke Mijnstreek': Harry Heykens, Jan Houwen, Yvonne Monse, Ria Claessens, Ine Janssen and Hanneke de Jongh. The municipality of Sittard-Geleen-Born: Berry van Rijswijk, Piet Veugen, Daphne Kagelmaker and Jack Ehlen. Other cooperating parties in the Sittard area: Health Insurance Companies, GPs, Maasland Hospital; Herbert Habets and Wilma Krämer, RIO and Associations for the Elderly. Research assistance: Annemarie Spaninks, Truus Custers, Marion Gijbels, Carla Verheggen and Marijke Moll. 


\section{References}

1. CBS [http://www.statline.cbs.nl]. Voorburg/Heerlen, 2000/2003.

2. van Haastregt JC, Diederiks JP, van Rossum E, de Witte LP, Crebolder HF. Effects of preventive home visits to elderly people living in the community: systematic review. BMJ 2000;320(7237):754-8.

3. Elkan R, Kendrick D, Dewey M, Hewitt M, Robinson J, Blair M, et al. Effectiveness of home based support for older people: systematic review and meta-analysis. BMJ Clin res ed 2001;323(7315):719-25.

4. Stuck AE, Egger M, Hammer A, Minder CE, Beck JC. Home visits to prevent nursing home admission and functional decline in elderly people: systematic review and meta-regression analysis. JAMA 2002;287(8):1022-8.

5. van Rossum E, Frederiks CM, Philipsen H, Portengen K, Wiskerke J, Knipschild P. Effects of preventive home visits to elderly people. BMJ 1993;307(6895):27-32.

6. van Rossum E. Effects of preventive home visits to the elderly. PhD Thesis Maastricht University, 1993.

7. Hall N. Randomized trial of a health promotion program for frail elders. Canadian Journal on Aging/La Revue Canadienne du vieillissement 1992;11(1):72-91.

8. Leveille SG, Wagner EH, Davis C, Grothaus L, Wallace J, LoGerfo M, et al. Preventing disability and managing chronic illness in frail older adults: a randomized trial of a community-based partnership with primary care.[comment]. J Am Geriatr Soc 1998;46(10):1191-8.

9. Pathy MS, Bayer A, Harding K, Dibble A. Randomised trial of case finding and surveillance of elderly people at home. Lancet 1992;340(8824):890-3.

10. Robichaud L, Hébert R, Roy PM, Roy C. A preventive program for community-dwelling elderly at risk of functional decline: a pilot study. Arch Gerontol Geriatr 2000;30:73-84.

11. Nikolaus T, Bach M. Preventing falls in community-dwelling frail older people using a home intervention team (HIT): results from the randomized Falls-HIT trial. J Am Geriatr Soc 2003;51(3):300-5.

12. Stuck AE, Minder CE, Peter Wuest I, Gillmann G, Egli C, Kesselring A, et al. A randomized trial of in-home visits for disability prevention in community-dwelling older people at low and high risk for nursing home admission. Archives of internal medicine 2000;160(7):97786.

13. van Haastregt JC, Diederiks JP, van Rossum E, de Witte LP, Voorhoeve PM, Crebolder HF. Effects of a programme of multifactorial home visits on falls and mobility impairments in elderly people at risk: randomised controlled trial.[comment]. BMJ 2000;321(7267):994-8.

14. Dalby DM, Sellors JW, Fraser FD, Fraser C, van Ineveld C, Howard M. Effect of preventive home visits by a nurse on the outcomes of frail elderly people in the community: a randomized controlled trial. CMAJ Canadian Medical Association journal journal de l'Association medicale canadienne 2000;162(4):497-500.

15. Randomization [http://www.randomization.com].

16. Hendriksen C, Lund E, Stromgard E. Consequences of assessment and intervention among elderly people: a three year randomised controlled trial. BMJ Clin res ed 1984;289(6457):1522-4.

17. Stuck AE, Zwahlen HG, Neuenschwander BE, Meyer Schweizer RA, Bauen G, Beck JC. Methodologic challenges of randomized controlled studies on in-home comprehensive geriatric assessment: the EIGER project. Evaluation of In-Home Geriatric Health Visits in Elderly Residents. Aging Milan, Italy 1995;7(3):218-23.

18. de Groen B. Disease management: The American Dream? Soesterberg: Stichting Zorgverlening Het Zonnehuis, 2001.

19. Yura $\mathrm{H}$, Walsh $\mathrm{M}$. The nursing proces: assessing, planning, implementing, evaluating. 5th edition ed. Connecticut/San Mateo, California: Appletton-Century-Crofts, 1988.

20. Richardson J. The Easy-Care assessment system and its appropriateness for older people. Nurs Older People 2001;13(7):17-9.

21. EASY-Care [http://www.bbr-online.com/easycare]. Sheffield. 
22. Podsiadlo D, Richardson S. The timed "Up \& Go": a test of basic functional mobility for frail elderly persons. J Am Geriatr Soc 1991;39(2):142-8.

23. Yesavage JA, Brink TL, Rose TL, Lum O, Huang V, Adey M, et al. Development and validation of a geriatric depression screening scale: a preliminary report. J Psychiatr Res 1982;17(1):37-49.

24. Folstein MF, Folstein SE, McHugh PR. "Mini-mental state". A practical method for grading the cognitive state of patients for the clinician. J Psychiatr Res 1975;12(3):189-98.

25. Carpenito L. Handbook of Nursing Diagnosis. 4th ed ed. Philadelphia: J.B. Lippincott Company, 1993.

26. Goossens ME, Rutten-van Molken MP, Vlaeyen JW, van der Linden SM. The cost diary: a method to measure direct and indirect costs in cost-effectiveness research. J Clin Epidemiol 2000;53(7):688-95.

27. Nicolaides AIE, van Rossum E, Spaninks AM, Kempen GI, Heykens H, Houwen J, et al. De gezondheidstoestand van zelfstandig wonende ouderen in Sittard en Geleen. Maastricht University, Gezondheidsstatistieken HQ 1064.L3, 2004.

28. Kempen GI, Doeglas DM, Suurmeijer TP. Het meten van problemen met zelfredzaamheid op verzorgend en huishoudelijk gebied met de Groningen Activiteiten Restrictie Schaal (GARS) - een handleiding. Groningen: Rijksuniversiteit Groningen, Noordelijk Centrum voor Gezondheidsvraagstukken, 1993.

29. RAND-36 [http://www.rand.org/health/surveys/sf36item].

30. Kempen GI, Brilman EI, Heyink JW, Ormel J. Het meten van de algemene gezondheidstoestand met de MOS Short-Form General health Survey (SF-20): een handleiding. Groningen: Noordelijk Centrum voor Gezondheidsvraagstukken, 1995.

31. Arrindell WA, Ettema JHM. SCL-90, Handleiding bij een multidimensionele psychopathologie-indicator. Lisse: Swets\&Zeitlinger BV, 1986.

32. Kempen GI, Brilman EI, Ormel J. De Mini-Mental State Examination: normeringsgegevens en een vergelijking van een 12- en 20-item versie in een steekproef ouderen uit de bevolking. Tijdschrift voor Gerontologie en Geriatrie 1995;26(4):163-72.

33. Pearlin LI, Schooler C. The structure of coping. J Health Soc Beh 1978;19(1):2-21.

34. Kempen GI, van Eijk LM. The psychometric properties of the SSL12-I, a short scale for measuring social support in the elderly. Social Indicators Research 1995;35:302-12.

35. de Jong Gierveld J, van Tilburg TG. Measuring personal experiences and emotions with questionnaires: a study of the properties of the Loneliness-scale in different research projects. In: de Jong Gierveld\&van der Zouwen, editor. De vragenlijst in sociaal onderzoek: Een confrontatie van onderzoekspraktijk en -methodiek. Deventer: van Loghum Slaterus, 1987: $67-83$. 


\title{
3
}

\section{Home visiting program for older people with health problems: process evaluation}

\author{
Ans Bouman ${ }^{1}$, Erik van Rossum ${ }^{2}$, Herbert Habets ${ }^{3}$, \\ Gertrudis Kempen ${ }^{2}$ and Paul Knipschild ${ }^{4}$
}

J Adv Nurs 2007, 58(5):425-435

1 Department of Epidemiology, Maastricht University, Maastricht, the Netherlands

2 Department of Health Care Studies, Maastricht University, Maastricht, the Netherlands

3 Maasland Hospital, Sittard, the Netherlands

4 Department of General Practice, Maastricht University, Maastricht, the Netherlands 


\section{Abstract}

Aim

This paper is a report of a process evaluation of the content, patient compliance and participant experiences of a home visiting program for older people with self-reported health problems.

\section{Background}

Systematic home visits by nurses to older people are a public health strategy to improve their functional abilities and prevent institutionalization. Evidence of the usefulness of these visits is conflicting, partly due to a lack of information on the intervention process in these programs.

\section{Method}

One hundred and sixty people (aged 70-84 years) participated in the program and were visited eight times during an 18-month period (February 2003-October 2004) by experienced home nurses. The nurses recorded key elements of each visit on standard forms: topics discussed, interventions regarding health problems and risks, and compliance with these interventions. Participants' and nurses' opinions on the visits were obtained through interviews at the end of the program.

\section{Findings}

Nearly $80 \%$ of the group received the complete program. Problems were detected in nearly all visits, mainly regarding social and psychological functioning, circulatory and musculoskeletal problems, and vision or hearing impairment. On average, 10 problems and 11 interventions were recorded per participant. Nearly $40 \%$ of interventions related to referrals, $45 \%$ to advice and $17 \%$ consisted of information-giving. The compliance rate was $65 \%$ for referrals and $58 \%$ for advice. Both nurses and participants were positive about the program.

\section{Conclusion}

A health visiting program, performed by home nurses, is feasible and appreciated by frail elders. The latter seem to be a relevant target population in view of the many and varied problems detected. 


\section{Introduction}

Preventive programs have been developed in the last two decades aimed at improving the independent functioning of older people and at reducing health care cost. One of such programs is home visitation by public health nurses to older people living in the community. In several countries, e.g., Denmark, Australia and Japan, these programs are part of national policy. Key elements of the (systematic) visits are: assessment of health problems and risks, advice, and referral to professional or community services.

\section{Background}

The results of trials on the effects of home visits have been inconsistent. ${ }^{1-4}$ Home visitations for a restricted population seems a more promising approach. This was demonstrated in five controlled studies, ${ }^{5-9}$ although three other trials did not support this assumption. ${ }^{10-12}$ A trial in the Netherlands $(n=580)$ showed that preventive home visits do not seem to be useful for the general population of elders, although a post hoc subgroup analysis indicated that the visits seemed to be effective for those with poor perceived health status at baseline. ${ }^{13} \mathrm{We}$ therefore decided to test this finding in a new randomized controlled trial, focusing entirely on older people with a poor health status. The trial was conducted in the Netherlands. The participants in the intervention group $(n=160)$ received at least eight home visits by home nurses over an 18-month period; the control group $(n=170)$ received usual care. Details of the trial design have been published elsewhere. ${ }^{14}$ The trial is not yet complete, and the effects on health status and care utilization will be reported upon completion.

This paper is a report of the process evaluation of the home visiting program. Such an evaluation is important in view of the inconsistent results so far on the effectiveness of the visits. Many factors play a role in the effectiveness of the program, including the target population, characteristics of the intervention, the people carrying out the visits and the compliance with the given advice. More insight into these factors is needed for introducing new programs into regular care and for evaluation research in the future.

The majority of trials on the effects of home visits provide only general information about the intervention program. In a systematic review of 33 publications reporting the results of 15 trials, van Haastregt et al. found that more than half of the reports did not describe protocol deviations, the completion of the intervention program, compliance to interventions, and the opinion of participants and home visitors about the program. ${ }^{15}$ They concluded that there is a serious lack of information on the intervention process in trials of preventive home visits. Similar shortcomings came forward in more recent trial reports. ${ }^{11} 1216-21$ Oakley and colleagues recently pointed out a general lack of information about the processes involved in implementing complex 
interventions in evaluation research, which hampers the valid interpretation of results and thereby the implementation of useful interventions. ${ }^{22}$

\section{The Study}

Aim

The aim of this study was to carry out a process evaluation of a home visiting program for older people with self-reported health problems, focusing on the following questions:

- What was the content of the visits, regarding discussed topics, treated problems and recommended interventions?

- What was the compliance with the given recommendations?

- What were the experiences of participants and nurses with the visiting program?

\section{Design and methods}

We conducted a randomized controlled trial to investigate the effects of a home visiting program. In this paper we focus on the intervention group, describing the process evaluation of the program. The home visits took place between February 2003 and October 2004.

\section{Participants}

The eligibility of older people to participate was determined through a questionnaire mailed to 4,901 people aged 70-84 living at home in a town in the south of the Netherlands (Sittard and surrounding areas). Their addresses were drawn from the population register of the municipality. The response rate was $76 \%$. The main inclusion criterion was a poor self-reported health status (scores $1-5$ on a scale ranging from 1-10 points). Excluded were those on a waiting list for a nursing home or a home for older people, as well as people already receiving home nursing care on a regular basis. Based on information from participants' general practitioners (GPs), we excluded the terminally ill. Finally, we randomly allocated 160 people to the intervention group and 170 to the control group.

\section{Intervention}

The program consisted of eight visits over a period of 18 months, and participants in the intervention group received a visit approximately every 2 months, always from the same nurse. If necessary, extra visits were made. The visits lasted between 60 and 90 minutes. The nurses followed a structured 
protocol to carry out the visits. We assigned each nurse to a number of GP practices. To inform the GPs, the treated problems, interventions and evaluations were mailed to them after completion of visits 1-3, 4-6 and 7-8.

\section{The nurses}

Three experienced home nurses from a local home care organization were appointed half time for the study and they conducted the visits under the supervision of a public health nurse (home nurses are auxiliary community nurses performing tasks under the supervision of a community nurse). The nurses recorded key elements on standard forms for each visit.

Each nurse was responsible for 51-57 persons. An experienced public health nurse supervised the home nurses at meetings held once every fortnight; where cases were discussed and attention was given to possible differences in approach between the nurses. The home nurses were not part of a multidisciplinary team, but advice could be obtained from specialists within the community care organization, e.g. a dietician, a diabetes specialist and an occupational therapist. A nurse gerontology specialist (author $\mathrm{HH}$ ) from the local hospital could also be consulted. At regular intervals, he advised the nurses on important gerontology issues.

\section{Visiting protocol}

The visits were conducted in a systematic way according to Yura and Walsh's nursing model which has four steps: diagnosis, planning of activities, carrying out the activities and evaluation. ${ }^{23}$ A number of elements, which seemed to be of importance for the content of the visits, were included in the protocol: e.g. face-to-face assessment, adequate communication between nurses and older people including an empathic attitude by the nurse, an individual plan, a clientcentred approach, follow-up of compliance with the given advice and multiple visits. ${ }^{324}$ In the 3-month period before the start of the visits, nurses were actively involved in the development of the visiting protocol. Several pilot visits were conducted in which different aspects of the protocol were tested. Relevant adaptations to the protocol were made before the start of the visiting program. The nurses also received relevant training in communication skills and using assessment tools.

\section{Diagnosis}

Our starting-point was a client-centred approach. The older people could indicate which problems they experienced and which needs they had. The EasyCare Questionnaire, an elderly assessment system, was used to detect further problems. ${ }^{25}$ Additional checklists could be used for a variety of topics: e.g. vision, hearing, mobility, cognitive status and depressive symptoms. During the visits no physical examination took place. If necessary, participants were referred to their GPs. On the basis of the problems indicated by the older people and those detected by the questionnaire and checklists, the nurses formulated identified problems and risks using a handbook of nursing diagnosis. ${ }^{26}$ 


\section{Planning activities}

A plan was set up for each client individually. The activities were planned in agreement with the older people. A limited number of problems were managed at one visit (a maximum of three problems and two interventions per problem). If necessary, nurses also used Carpenito's handbook to select interventions. ${ }^{26}$

\section{Carrying out the activities}

The participants themselves were primarily responsible for carrying out the planned activities. The home nurse only supported them. In order to improve compliance, the nurses contacted the older people by telephone 1-4 weeks after each visit, depending on the type of advice. They verified if the recommendations had been applied and, if not, what the impediments were and whether further assistance was necessary. The nurses were available by telephone in case of problems or if more information was needed.

\section{Evaluation}

The evaluation of each home visit took place at the next visit. The cycle was then repeated and new or old, but not solved, problems could be dealt with. After the intervention period, individual structured interviews were held with participants and nurses: they were asked about their experiences and expectations of the program.

Nurses recorded each visit immediately afterwards on structured registration forms: time spent on the visit, the three topics they spent most of the time on, treated problems, referral to other services, advice or information given, and compliance with recommendations made during the previous visit (including reasons for noncompliance). For the last visit compliance was evaluated afterwards by telephone. A research assistant checked fortnightly whether the registration forms were filled in completely. The extent to which the program was performed according to the protocol was monitored by one of the researchers $(\mathrm{AB})$ during fortnightly meetings with the nurses.

The evaluation of the participants' experiences with the visits included their enjoyment of the visits, their opinion about the duration and the number of visits and their perceived benefits of the visits. The data were collected by means of individual interviews conducted by independent interviewers at the end of the intervention period.

The nurses' experiences with the visiting program included their opinions about feasibility, general aspects of the program, the visiting protocol and the meetings with the supervising nurse. The assessment took place at the end of the intervention period by means of individual interviews conducted by one of the researchers $(\mathrm{AB})$. In addition, the nurses recorded the expected effectiveness of the program on the quality of life for each participant. Table 1 summarizes the aspects of the process evaluation and methods of data collection. 
Table 1 Evaluation of the visiting program

Aspect Method

During the program

- number of visits and time spent

recorded by the nurses on

- discussed topics

- treated problems and recommendations

registration forms; after each visit

- compliance

- protocol deviations

qualitative data; at fortnightly meetings with the nurses

After the program

Opinion of participants about the program

- enjoyment of the visits

- general aspects of the visits

- compliance

- perceived benefits

Opinion of nurses about the program

- feasibility

- general aspects of the visits

- visiting protocol

- meetings with the supervising nurse

- expected effectiveness of the

individual interviews by independent interviewers; after the last visit

individual interviews by one of the researchers; after the end of the program program for each participant

recorded by the nurses on registration forms; after the last visit

\section{Ethical considerations}

We obtained the approval of the Medical Ethics Committee of Maastricht University/Academic Hospital Maastricht. Information for patients about the aim of the study, procedures, protection of privacy, and insurance cover (as required by law), was included with the questionnaire. Return of a completed and signed informed consent form (included) was taken as consent to participate.

\section{Data analysis}

The data were analysed descriptively, using univariate and bivariate analyses. The analyses were conducted using the Statistical Package for the Social Sciences (SPSS, SPSS Inc., Chicago, IL, USA). 


\section{Results}

\section{Participants}

Table 2 gives baseline characteristics of the 160 participants. The mean age was 76 years $(\mathrm{SD}=3.7)$. About half of this population had at least one Activities of Daily Living (ADL) dependency and/or two Instrumental Activities of Daily Living (IADL) dependencies. Nearly half of the population indicated that their health had deteriorated in the 3 months before the start of the visiting program.

\section{Protocol deviations}

During the program, nurses reported no substantial protocol deviations at the meetings with the research team. Minor deviations related to spreading the comprehensive baseline assessment over the first two visits because of time shortage (all visited participants) and waived follow-up telephone calls after the visits (11 older people indicated that this was not necessary).

\section{Number and duration of visits}

Eighteen participants died just before or during the intervention period (11\%) and 18 withdrew from the program $(11 \%)$, due to illness $(n=9)$, lack of interest $(\mathrm{n}=5)$ or lack of time $(\mathrm{n}=4)$. In total, 151 participants $(95 \%)$ received visits; 124 (78\%) received the complete program of eight visits and $27(17 \%)$ received, on average, four visits (see Figure 1).

The total number of visits was 1,107, an average of seven visits per person. If possible, the visits were continued when participants were admitted to a home for older persons (nine visits, $n=3$ ) or a nursing home (three visits, $n=3$ ). Those visits were included in the data set. The number of follow-up telephone calls was 985 , that is, an average of six calls per person. 
Table 2 Baseline characteristics of the participants

\begin{tabular}{|c|c|c|c|}
\hline \multicolumn{2}{|l|}{ Characteristic } & \multicolumn{2}{|c|}{$\begin{array}{l}\text { Participants } \\
\qquad(\mathrm{n}=160)\end{array}$} \\
\hline \multicolumn{2}{|c|}{ Age, mean \pm standard deviation } & 75.8 & (3.7) \\
\hline \multicolumn{4}{|l|}{ Sex, n (\%) } \\
\hline \multicolumn{2}{|l|}{ male } & 64 & $(40)$ \\
\hline \multicolumn{2}{|l|}{ female } & 96 & $(60)$ \\
\hline \multicolumn{4}{|c|}{ Household Composition, n (\%) } \\
\hline \multicolumn{2}{|l|}{ alone } & 53 & (34) \\
\hline \multicolumn{2}{|l|}{ together } & 103 & $(66)$ \\
\hline \multicolumn{4}{|l|}{ Education, n (\%) } \\
\hline \multicolumn{2}{|l|}{ primary school } & 60 & (39) \\
\hline \multicolumn{2}{|c|}{ lower to middle professional education } & 81 & $(52)$ \\
\hline \multicolumn{2}{|c|}{ higher professional education } & 15 & $(10)$ \\
\hline \multicolumn{4}{|l|}{ Self-rated health*, n (\%) } \\
\hline \multicolumn{2}{|l|}{$1-4$} & 62 & $(39)$ \\
\hline \multicolumn{2}{|l|}{5} & 98 & (61) \\
\hline \multicolumn{4}{|l|}{ Functional status $^{\dagger}, \mathrm{n}(\%)$} \\
\hline \multirow[t]{2}{*}{ ADL-dependencies } & 0 & 73 & $(46)$ \\
\hline & $1-11$ & 86 & $(54)$ \\
\hline \multirow[t]{2}{*}{ IADL-dependencies } & $0-1$ & 76 & $(49)$ \\
\hline & $2-7$ & 79 & $(51)$ \\
\hline \multicolumn{4}{|c|}{ Health change in previous 3 months, $n(\%)$} \\
\hline \multicolumn{2}{|l|}{ same or better } & 85 & $(53)$ \\
\hline \multicolumn{2}{|l|}{ worse } & 75 & $(47)$ \\
\hline \multicolumn{4}{|c|}{ Health affects social participation, n (\%) } \\
\hline \multicolumn{2}{|l|}{ often } & 82 & $(53)$ \\
\hline \multicolumn{2}{|l|}{ sometimes } & 49 & $(32)$ \\
\hline \multicolumn{2}{|l|}{ never } & 24 & $(16)$ \\
\hline \multicolumn{4}{|c|}{ Contacted general practitioner in previous } \\
\hline \multicolumn{2}{|c|}{$\begin{array}{l}\text { Contacted medical specialist in previous } \\
3 \text { months, n (\%) }\end{array}$} & 108 & (69) \\
\hline \multicolumn{2}{|l|}{ Use of home care, $\mathrm{n}(\%)$} & 64 & $(40)$ \\
\hline
\end{tabular}

Note: ADL or IADL-dependencies indicate the number of activities for which the older people are dependant on others to perform the activity.

* Indicated by a score on a scale ranging from 1-10 points.

Participants with poor health status (score <6) were included.

$\dagger$ Refers to 11 activities of daily living (ADLs) and seven instrumental (household) activities of daily living (IADLs). 


\section{Chapter 3}

The total time spent on each of the visits was, on average, 114 minutes. This included the average time spent on the visits in-home (65 minutes, $\mathrm{SD}=11.8$ ), preparation time (9 minutes, $\mathrm{SD}=2.7)$, travel time $(17$ minutes, $\mathrm{SD}=9.9)$, administration time (15 minutes, $\mathrm{SD}=10.7)$ and follow-up telephone call (8 minutes, $\mathrm{SD}=3.4$ ). The average time spent on the first visit (in-home) was 83 minutes $(\mathrm{SD}=12.0)$, on the second 66 minutes $(\mathrm{SD}=11.3)$, while the average time ranged from 60-63 minutes for visits 3-8.

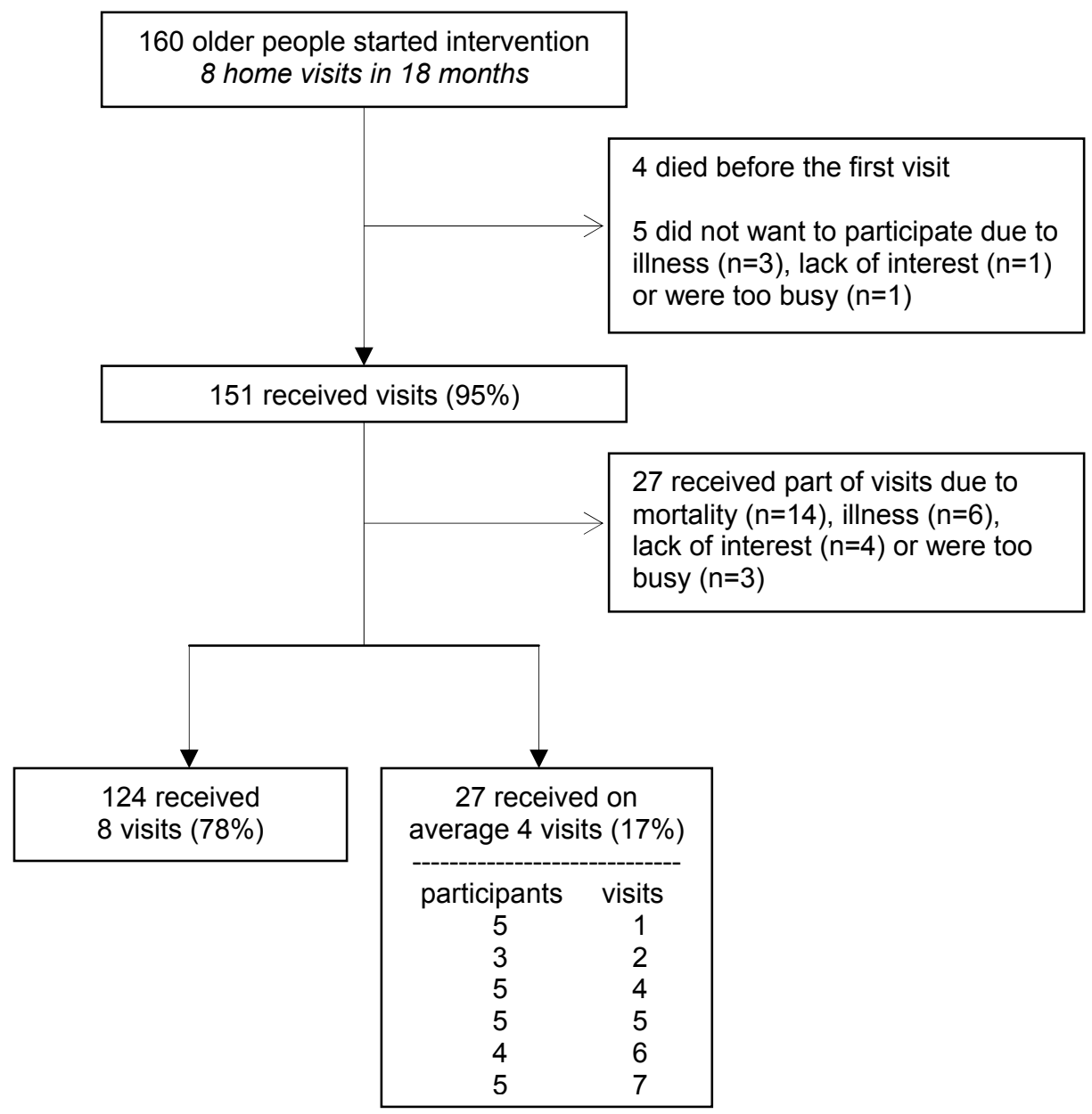

Figure 1 Flow diagram of the participants receiving home visits 


\section{Topics discussed}

After each visit, nurses recorded a maximum of three topics they spent most of the time on. In total, 3299 topics were recorded. These were divided into 10 categories (see Table 3). The two most frequently recorded categories were psychosocial functioning (social contacts and mental status, 21\%) and functional status $(20 \%)$, of which mobility was the most discussed topic. General health status accounted for $17 \%$ of the recorded topics.

Table 3 Topics discussed during the intervention

\begin{tabular}{|c|c|c|}
\hline Topic & Number & Percentage \\
\hline Psychosocial functioning & 693 & $21 \%$ \\
\hline social contacts & 414 & $12 \%$ \\
\hline mental status & 279 & $9 \%$ \\
\hline Functional status & 630 & $20 \%$ \\
\hline mobility & 450 & $14 \%$ \\
\hline daily care & 93 & $3 \%$ \\
\hline incontinence & 87 & $3 \%$ \\
\hline General health status & 555 & $17 \%$ \\
\hline Physical condition & 340 & $10 \%$ \\
\hline vision/hearing & 157 & $5 \%$ \\
\hline leg/feet & 145 & $4 \%$ \\
\hline dentures & 38 & $1 \%$ \\
\hline Health services & 298 & $9 \%$ \\
\hline Nutrition & 201 & $6 \%$ \\
\hline Medication & 176 & $5 \%$ \\
\hline Sleep & 127 & $4 \%$ \\
\hline Life events & 122 & $4 \%$ \\
\hline Other & 157 & $5 \%$ \\
\hline Total & 3,299 & $100 \%$ \\
\hline
\end{tabular}

\section{Problems treated}

Table 4 presents the number of participants for whom a problem was identified and an intervention recommended, by problem category. Based on Carpenito's handbook, 28 problem categories (not tabulated) were recorded and these were coded according to the International Classification of Primary Care (ICPC), with ADL/IADL as a separate category. ${ }^{26} 27$ 
Chapter 3

Table 4 Number and percentage of treated problems, interventions and compliance

Problem categories $^{27} \quad$ Participants, $n(\%) \quad$ Problems

\begin{tabular}{|c|c|c|c|}
\hline Health in general & 132 & $(87 \%)$ & $\begin{array}{c}408 \quad(26 \%) \\
2.7 \mathrm{pp}^{\ddagger}\end{array}$ \\
\hline \multirow[t]{2}{*}{ Psychological } & 100 & $(66 \%)$ & $239 \quad(16 \%)$ \\
\hline & & & $1.6 \mathrm{pp}$ \\
\hline \multirow[t]{2}{*}{ Musculoskeletal } & 95 & $(63 \%)$ & $220 \quad(14 \%)$ \\
\hline & & & $1.5 \mathrm{pp}$ \\
\hline \multirow[t]{2}{*}{ Social } & 79 & $(52 \%)$ & $(10 \%)$ \\
\hline & & & $1.0 \mathrm{pp}$ \\
\hline \multirow[t]{2}{*}{ Circulatory } & 76 & $(50 \%)$ & 141 \\
\hline & & & $0.9 \mathrm{pp}$ \\
\hline \multirow[t]{2}{*}{ Eye/ear } & 71 & $(47 \%)$ & 113 \\
\hline & & & $0.7 \mathrm{pp}$ \\
\hline \multirow{2}{*}{$\begin{array}{l}\text { Nutritional/ } \\
\text { digestive }\end{array}$} & 62 & $(41 \%)$ & $(6 \%)$ \\
\hline & & & $0.6 \mathrm{pp}$ \\
\hline \multirow[t]{2}{*}{ ADL/IADL } & 57 & $(38 \%)$ & $(6 \%)$ \\
\hline & & & $0.6 \mathrm{pp}$ \\
\hline \multirow[t]{2}{*}{ Urinary } & 48 & $(32 \%)$ & $(5 \%)$ \\
\hline & & & $0.5 p p$ \\
\hline \multirow[t]{2}{*}{ Other } & 15 & $(10 \%)$ & $(1 \%)$ \\
\hline & & & $0.1 \mathrm{pp}$ \\
\hline \multirow[t]{2}{*}{ Total } & 151 & $(100 \%)$ & \multirow{2}{*}{$1,545(100 \%)$} \\
\hline & & & \\
\hline
\end{tabular}

Notes: ADL, Activities of daily living; IADL, Instrumental (household) activities of daily living.

* Services referred to are presented in Table 5.

$\dagger$ Examples of given advice are presented in the text.

$\ddagger$ pp: per person of total group $(n=151)$.

$\S$ The information (i) given during the visits was not evaluated on compliance, because this did not include clear recommendations for the participant. 
Table 4 continued

\begin{tabular}{|c|c|c|c|c|}
\hline Interventions & \multicolumn{2}{|c|}{$\begin{array}{l}\text { referrals }(r)^{*} \\
\text { advice }(a)^{\dagger} \\
\text { info (i) }\end{array}$} & \multicolumn{2}{|c|}{ Complied $^{\S}$} \\
\hline $\begin{array}{c}476 \quad(27 \%) \\
3.2 \mathrm{pp}^{\ddagger}\end{array}$ & $\begin{array}{ll}\text { r: } & 152 \\
\text { a: } & 219 \\
\mathrm{i}: & 105\end{array}$ & $\begin{array}{l}(32 \%) \\
(46 \%) \\
(22 \%)\end{array}$ & $\begin{array}{ll}\mathrm{r}: & 101 \\
\mathrm{a}: & 130\end{array}$ & $\begin{array}{l}(66 \%) \\
(59 \%)\end{array}$ \\
\hline $268 \quad(15 \%)$ & $\begin{array}{ll}\mathrm{r}: & 78 \\
\mathrm{a}: & 116 \\
\mathrm{i}: & 74\end{array}$ & $\begin{array}{l}(29 \%) \\
(43 \%) \\
(28 \%)\end{array}$ & $\begin{array}{ll}\text { r: } & 45 \\
\text { a: } & 68\end{array}$ & $\begin{array}{l}(58 \%) \\
(59 \%)\end{array}$ \\
\hline $249{ }_{1.6 p p}^{(14 \%)}$ & $\begin{array}{ll}\text { r: } & 112 \\
\text { a: } & 107 \\
\mathrm{i}: & 30\end{array}$ & $\begin{array}{l}(45 \%) \\
(43 \%) \\
(12 \%)\end{array}$ & $\begin{array}{ll}\mathrm{r}: & 88 \\
\mathrm{a}: & 59\end{array}$ & $\begin{array}{l}(79 \%) \\
(55 \%)\end{array}$ \\
\hline $\begin{array}{l}157 \quad(9 \%) \\
1.0 \mathrm{pp}\end{array}$ & $\begin{array}{ll}\mathrm{r}: & 51 \\
\mathrm{a}: & 72 \\
\mathrm{i}: & 34\end{array}$ & $\begin{array}{l}(32 \%) \\
(46 \%) \\
(22 \%)\end{array}$ & $\begin{array}{ll}\mathrm{r}: & 36 \\
\mathrm{a}: & 38\end{array}$ & $\begin{array}{l}(71 \%) \\
(53 \%)\end{array}$ \\
\hline $\begin{array}{l}160 \\
1.1 \mathrm{pp}\end{array}$ & $\begin{array}{ll}\text { r: } & 44 \\
\text { a: } & 97 \\
\text { i: } & 19\end{array}$ & $\begin{array}{l}(28 \%) \\
(61 \%) \\
(12 \%)\end{array}$ & $\begin{array}{ll}\mathrm{r}: & 31 \\
\mathrm{a}: & 57\end{array}$ & $\begin{array}{l}(70 \%) \\
(59 \%)\end{array}$ \\
\hline $\begin{array}{l}120 \\
0.8 \mathrm{pp}\end{array}$ & $\begin{array}{ll}\text { r: } & 91 \\
\text { a: } & 22 \\
\text { i: } & 7\end{array}$ & $\begin{array}{r}(76 \%) \\
(18 \%) \\
(6 \%)\end{array}$ & $\begin{array}{ll}\mathrm{r}: & 41 \\
\mathrm{a}: & 13\end{array}$ & $\begin{array}{l}(45 \%) \\
(59 \%)\end{array}$ \\
\hline $\begin{array}{cc}104 & (6 \%) \\
0.7 \mathrm{pp} & \end{array}$ & $\begin{array}{ll}\text { r: } & 50 \\
\text { a: } & 45 \\
\text { i: } & 9\end{array}$ & $\begin{array}{r}(48 \%) \\
(43 \%) \\
(9 \%)\end{array}$ & $\begin{array}{ll}\text { r: } & 26 \\
\text { a: } & 28\end{array}$ & $\begin{array}{l}(52 \%) \\
(62 \%)\end{array}$ \\
\hline $\begin{array}{ll}104 & (6 \%) \\
0.7 \mathrm{pp} & \end{array}$ & $\begin{array}{ll}\text { r: } & 49 \\
\text { a: } & 39 \\
\text { i: } & 16\end{array}$ & $\begin{array}{l}(47 \%) \\
(38 \%) \\
(15 \%)\end{array}$ & $\begin{array}{ll}\text { r: } & 40 \\
\text { a: } & 21\end{array}$ & $\begin{array}{l}(82 \%) \\
(54 \%)\end{array}$ \\
\hline $\begin{array}{l}77 \quad(4 \%) \\
0.5 \mathrm{pp}\end{array}$ & $\begin{array}{ll}\mathrm{r}: & 14 \\
\mathrm{a}: & 59 \\
\mathrm{i}: & 4\end{array}$ & $\begin{array}{r}(18 \%) \\
(77 \%) \\
(5 \%)\end{array}$ & $\begin{array}{ll}\mathrm{r}: & 8 \\
\mathrm{a}: & 35\end{array}$ & $\begin{array}{l}(57 \%) \\
(59 \%)\end{array}$ \\
\hline $190.1 \mathrm{pp}$ & $\begin{array}{ll}\mathrm{r}: & 9 \\
\mathrm{a}: & 10 \\
\mathrm{i}: & 0\end{array}$ & $\begin{array}{r}(47 \%) \\
(53 \%) \\
(0 \%)\end{array}$ & $\begin{array}{ll}\mathrm{r}: & 8 \\
\mathrm{a}: & 9\end{array}$ & $\begin{array}{l}(89 \%) \\
(90 \%)\end{array}$ \\
\hline $\begin{array}{c}1,734 \quad(100 \%) \\
11.5 \mathrm{pp}\end{array}$ & $\begin{array}{ll}\text { r: } & 650 \\
\text { a: } & 786 \\
\text { i: } & 298\end{array}$ & $\begin{array}{l}(38 \%) \\
(45 \%) \\
(17 \%)\end{array}$ & $\begin{array}{ll}\mathrm{r}: & 424 \\
\mathrm{a}: & 458\end{array}$ & $\begin{array}{l}(65 \%) \\
(58 \%)\end{array}$ \\
\hline
\end{tabular}

At each visit nurses could, for reasons of feasibility, treat a maximum of three problems. In $91 \%$ of all visits, nurses treated one or more problems. In the remaining visits, problems were identified, but no recommendations were given (not tabulated). In those visits, an informal 'talk' about the problem was considered sufficient by the older people or there was no treatment available. On average, there were 10 treated problems per participant over 18 months. 
Problems were identified and treated in all major gerontology areas. One out of four problems concerned 'health in general'; $87 \%$ had problems in this category (Table 4). This category includes (not tabulated): therapy discipline (4\%), risk of injury (4\%), pain ( $4 \%)$, general deterioration $(2 \%)$ and other health problems $(12 \%)$. The second largest category was 'psychological problems' (16\%). Two out of three participants reported problems in this category, predominantly (not tabulated) mental (10\%); sleep and memory problems each accounted for $3 \%$. Six percent of the problems were related to self-care and household activities; $38 \%$ of older people reported problems in this category.

\section{Interventions}

Table 4 also presents the interventions by problem category. For each problem a maximum of two interventions could be recorded (for reasons of feasibility). On average, 11 interventions per participant were recorded. Each intervention could be categorized into referrals to other services or health carers, advice, and information on a specific problem. Of all interventions, 38\% were referrals, $45 \%$ advice and $17 \%$ information. For example, three out of four interventions $(76 \%)$ concerning eye and ear problems were referrals, while interventions on urinary tract problems were mostly advice ( $77 \%$, usually to obtain aids).

Table 5 presents data on referrals. Nearly all participants (95\%) were at least once referred to other services and, on average, four referrals were given per person. A substantial part was referrals to the GP: during the program $84 \%$ of the participants were, on average, twice advised to see their GP. Reasons for referral to the GP were, for example, to discuss functional problems, to ask for a treatment or to examine the use of multiple medications prescribed by different medical doctors (not tabulated). Many referrals were also made to the local authorities, because municipalities in the Netherlands supply a number of aids. These accounted for $15 \%$ of referrals and were mostly related to applications for home aids (e.g. chair lift) or aids in transport (e.g. scoot mobiles). Home nursing care and home help accounted for $13 \%$ of the referrals.

\section{Compliance}

The compliance with referrals was $65 \%$ and with advice $58 \%$, the overall compliance being $61 \%$. The participants had complied with the referrals when they had contacted the services (and applications were made), and, in the case of advice, when they had completely adhered to the recommendations.

Per problem category, the compliance with referrals ranged from $45 \%$ to $82 \%$, and with advice from $53 \%$ to $62 \%$ (except 'other', see Table 4 ). The compliance was highest for referrals related to ADLs/IADLs (82\%) and musculoskeletal problems (79\%). A low compliance was observed for referrals related to eye and ear (45\%) and nutrition (52\%) problems. The compliance rate for advice varied less: the highest rate was for nutrition $(62 \%)$ and the lowest for social problems $(53 \%)$. 
The compliance by type of referral (Table 5) was highest for referrals to the local authorities ( $82 \%$, for several aids) and lowest for contacting an optician or audiologist (38\%). Nearly $70 \%$ of the participants contacted their GP, if the nurse recommended this. The main reasons for noncompliance, according to the nurses, were (not tabulated): participants wanted to postpone the planned activity $(n=21)$ or thought it was unnecessary $(n=17)$, illness $(n=14)$, were too busy $(\mathrm{n}=12)$ or said to have forgotten it $(\mathrm{n}=6)$.

Table 5 Number and percentage of referrals to health care and social services and compliance

\begin{tabular}{lrrrrrr}
\hline & \multicolumn{2}{c}{$\begin{array}{c}\text { Participants } \\
\mathrm{n}^{*}\end{array}$} & \multicolumn{2}{l}{ Referrals } & \multicolumn{2}{l}{ Complied } \\
& 127 & $(84 \%)$ & 251 & $(39 \%)$ & 172 & $(69 \%)$ \\
\hline General practitioner & 69 & $(46 \%)$ & 100 & $(15 \%)$ & 82 & $(82 \%)$ \\
$\begin{array}{l}\text { Local authorities (for aids)/ } \\
\text { housing }\end{array}$ & 54 & $(36 \%)$ & 83 & $(13 \%)$ & 65 & $(78 \%)$ \\
$\begin{array}{l}\text { Home nursing care/ home } \\
\text { help/ daycare }\end{array}$ & 52 & $(34 \%)$ & 66 & $(10 \%)$ & 25 & $(38 \%)$ \\
$\begin{array}{l}\text { Optician/ audiologist } \\
\text { Medical specialist }\end{array}$ & 40 & $(26 \%)$ & 52 & $(8 \%)$ & 34 & $(65 \%)$ \\
$\begin{array}{l}\text { Dietician/ 'meals on } \\
\text { wheels' }\end{array}$ & 37 & $(25 \%)$ & 45 & $(7 \%)$ & 20 & $(44 \%)$ \\
Physiotherapist & 18 & $(12 \%)$ & 23 & $(4 \%)$ & 12 & $(52 \%)$ \\
Social services & 12 & $(8 \%)$ & 12 & $(2 \%)$ & 7 & $(58 \%)$ \\
Dentist & 8 & $(5 \%)$ & 10 & $(2 \%)$ & 4 & $(50 \%)$ \\
Chiropodist & 8 & $(5 \%)$ & 8 & $(1 \%)$ & 3 & $(38 \%)$ \\
Total & 144 & $(95 \%)$ & 650 & $(100 \%)$ & 424 & $(65 \%)$ \\
\hline
\end{tabular}

* Number of referred participants.

$\dagger$ Percentage of total group $(n=151)$.

\section{Opinions on visits}

\section{Participants}

At the end of the program, 124 participants were interviewed about their opinion of the visits. A large majority reported that they had enjoyed the visits (89\%), all important matters had been discussed (90\%), they could bring forward their own wishes $(93 \%)$, and they were satisfied with the length of time $(83 \%)$ and the number of visits $(85 \%)$. Nearly all $(96 \%)$ considered the home nurses (very) competent. 
Another question concerned the perceived benefits of the visits. A substantial proportion of participants $(66 \%)$ thought they had greatly benefited, $23 \%$ reported benefits to some extent; and $11 \%$ reported they had not benefited at all. The main reported beneficial elements were: advice in general $(n=47)$, advice on aids and devices $(n=29)$, and the opportunity to discuss problems with the nurse $(n=22)$. About $60 \%$ of the older people indicated that they would participate again if a home visiting program was offered to them, while $25 \%$ probably would not (not tabulated).

\section{Nurses}

The experiences of the nurses with the program were in general positive. The first visits had been highly demanding for them, mainly because many and complex health problems came forward. They considered the visiting protocol very useful and appreciated the clear structure that it provided. For some participants, the nurses thought that five or six visits would have been sufficient. The telephone calls between the visits were considered valuable as these improved the contact between nurses and participants, and reminded the participant to carry out the planned activities. The fortnightly meetings were also considered valuable. Each nurse could discuss her cases with the supervising nurse and other nurses and receive feedback on how she managed the problems. Overall, nurses thought that the visits were useful in improving the independence of the older people and to (emotionally) support and advise them on a wide range of topics.

The nurses judged the effectiveness of the visiting program for each individual participant. They expected the program to be effective (or to be beneficial) for half of the participants (48\%); for $28 \%$ they were uncertain about possible effects and they thought there was no effect for one out of four participants $(24 \%)$. The nurses indicated that those who did not benefit were less open to change, did not understand or had little knowledge of the relevant problems, were less motivated and considered the relationship between nurse and participant not as optimal (not shown).

\section{Discussion}

\section{Study limitations}

We have reported the content, feasibility and acceptability of a home visiting program for frail elders, that is, people with a poor self-reported health status. This evaluation has some limitations. Firstly, most data were collected by the visiting nurses. Their recording of problems, interventions and compliance may have been biased to some extent, because it would be in the interest of the nurses to demonstrate a successful program. Blinding of these measurements is, however, hardly possible for practical and financial reasons. Also, the nurses needed to record most of these data themselves, because they needed them for 
managing each case. Secondly, it was possible that participants gave socially desirable answers to the nurses, for example, when evaluating compliance. Participants may have been more positive about their compliance in order not to disappoint the nurse. The same holds for their overall opinion on the visits, despite the fact that these data were gathered by independent interviewers. This can also possibly explain (part of) the disagreement in perceived benefits from the program between nurses and participants.

\section{Discussion of findings}

The program was largely carried out according to plan. A majority of participants $(78 \%)$ completed the entire program of eight visits. Of those who died or withdrew from the program, 53\% still received at least half of the planned visits. The number of withdrawals was low and often due to (severe) illness of the older people. The average duration of the visits (in-home) was 65 minutes, according to plan. The home nurses were able to perform the visits without absence owing to illness or any other impediments. No substantial deviations from the protocol were reported by nurses.

The process evaluation focused on three questions. Firstly, we made an assessment of the content of the visits. A broad range of topics came forward during the visits, including physical, mental as well as social problems. There was a continuous yield of problems throughout the program. In nearly all visits (91\%), nurses recorded problems and gave recommendations. In about half the population, problems were detected regarding social and psychological functioning, circulatory and musculoskeletal problems and vision or hearing impairments. On average, 10 problems and 11 interventions were recorded per participant over the 18 -month period. Nearly $40 \%$ of all interventions related to referrals, $45 \%$ to advice and $17 \%$ consisted of information given. Many referrals were to the GP (39\%). Secondly, the compliance with interventions was assessed: $65 \%$ of the older people complied with the referrals and $58 \%$ with the advice given by nurses. Thirdly, we assessed the experiences with and acceptability of the program. This showed that both nurses and participants were (very) positive about the program. A substantial proportion of participants thought they had greatly benefited from the visits $(66 \%)$. The nurses were somewhat less optimistic and estimated that $48 \%$ of the population had benefited from the program.

Only a small number of trials of the effects of home visits reported detailed results of a process evaluation. The major problems we identified are not different from those reported in other home visiting programs for older people. ${ }^{16}{ }^{28-30}$ Variations were shown between trials in the number of treated participants and interventions. In the trial by van Rossum, among the general population, half of the participants had problems identified and there were, on average, two reported interventions per person (over 3 years, on average 10 visits). ${ }^{28}$ In a study by Stuck et al., also among the general population, (nearly) all participants had problems identified and, on average, there were 29 
interventions per person (over 3 years, on average 11 visits). ${ }^{29} 31$ In our study, all participants had problems identified and there were 11 interventions per person (over 1.5 years, on average seven visits). Because we selected older people with poor self-reported health status, it was not surprising that all our participants had problems identified. The nurses in our study formulated the recommendations in agreement with the participants and the number was limited by the visiting protocol. The nurses in the trial by Stuck et al. formulated the recommendations in consultation with a geriatrician, which may have resulted in more recommendations. This may partly explain the differences in the number of interventions.

The compliance with referrals in the present study $(65 \%)$ is similar to that reported by van Rossum et al. ${ }^{13}$ Lower overall compliance rates were reported in other home visiting studies, ranging from $46 \%,{ }^{30} 47 \%,{ }^{20}{ }^{29-31}$ to $49 \%{ }^{20}$ The nurses may have improved overall compliance in our study (61\%) by reminding and supporting the participants by means of a telephone follow-up contact after each visit. Also, the planned activities were set up in agreement with the older people and the number of planned activities was kept low, so they would not be discouraged in following the advice. It is possible that the somewhat higher compliance with the referrals (65\% versus $58 \%$ for advice) is because this usually involved a one-time action by the participants. Advice often required the older people to change their life-style, which is far more difficult to establish. Alessi et al. also found higher compliance rates for referrals (51\%) than for selfcare activities such as changes in physical activity and diet $(30 \%){ }^{29}$ A low compliance was observed, however, in this study for eye and ear problems $(38 \%)$ even though this often involved a one-time action. In a study measuring hearing loss for subjects aged 85 years, it was found that the majority of older people declines auditory rehabilitation, ${ }^{32}$ the use of a hearing aid was not perceived as necessary by the older people in order to function on a daily basis. It is possible that this was also true for our participants and may partly explain the low compliance for eye and ear problems.

The critical role of patient adherence in successful health care outcomes was shown in a Japanese study, where home visits were found to be effective for a subgroup of elders who had complied with the advice, but not for the total group of those receiving recommendations. ${ }^{20}$

Both nurses and participants were satisfied with the program. Other studies also reported that $90 \%$ or more of the participants had enjoyed or appreciated the visits. ${ }^{28-3133}$ Fewer older people (about $60 \%$ in our study and $70 \%$ in the trial by van Rossum $^{28}$ ) indicated that they would participate again if a home visiting program was offered to them. This seems somewhat in contrast with the high proportion of satisfied elders. It is possible that some of the participants thought that the number of visits they had received was sufficient and there was no need to continue. Most people (85\%) in our study indicated that they were satisfied with the number of visits. Nurses also reported that, for a small group, five or six visits seemed to be sufficient. 
Although the participants were satisfied with the program, this does not necessarily mean that the program is also effective and should be implemented. In two earlier studies in the Netherlands, it was shown that no relationship exists between satisfaction with the program and its effectiveness. ${ }^{1528}$ Effectiveness is a necessary condition for implementation in regular care.

A majority of older people (66\%) reported that they had greatly benefited from the visits, while the nurses were less optimistic. Similar results were found by van Haastregt et al. ${ }^{30}$ The main reason why the participants experienced the visits in this study as beneficial was because of the advice they received. Another reason which came forward was that they were happy to be able to discuss problems with the nurse. Apart from possible socially desirable answers and the use of different answering categories for participants and nurses, the social aspect of the visits may partly explain the disagreement between the nurses and the participants as to the perceived benefits of the visits.

For implementation of the program the following elements should be taken into account. Home nurses, who are qualified at a lower professional level than public health nurses, are able to carry out the visits, but the visits can be demanding especially in the beginning. A preparatory period, including pilot visits, must be taken into account. The nurses referred many participants to their GP and attention should therefore be given to the communication between nurses and GPs. Traditionally, in the Netherlands, there is little contact between home care organizations and GPs. Because referrals are an important element of the visits, it is relevant that the nurses are acquainted with the health and social services for elders in the area. Depending on the needs of the older people, a more flexible visiting scheme may be considered.

\section{Summary statement}

\section{What is already known about this topic}

- Evidence of the effectiveness of preventive home visits to older people is conflicting.

- There is lack of information on the contents and implementation of interventions in home visiting trials; this hampers the interpretation of research findings, implementation of programs and future research in this field.

\section{What this paper adds}

- A health visiting program delivered by home nurses, under supervision of a public health nurse and targeted on older people with poor self-reported health, was feasible and was appreciated by both participants and nurses.

- Throughout an 18-month period, many and varied health problems were detected. 
Chapter 3

- Further attention is needed to improve the compliance of older people with advice about health problems and risks.

\section{Conclusion}

This process evaluation adds to the information about home intervention programs. The description and experiences of the program may support nurses in implementing such a program in regular care. Depending on the outcome of the effect study, further investigation may be needed into which elements of the program are most beneficial for older people.

\section{Author contributions}

$\mathrm{AB}, \mathrm{ER}, \mathrm{GK}$ and $\mathrm{PK}$ were responsible for the study conception and design and drafting of the manuscript. $\mathrm{AB}$ performed the data collection and $\mathrm{AB}$ and $\mathrm{ER}$ performed the data analyses. ER and PK obtained funding and $\mathrm{HH}$ provided administrative support. AB, ER, HH, GK and PK made critical revisions to the manuscript. ER, GK and PK provided statistical expertise and supervised the study. 


\section{References}

1. van Haastregt JC, Diederiks JP, van Rossum E, de Witte LP, Crebolder HF. Effects of preventive home visits to elderly people living in the community: systematic review. BMJ 2000;320(7237):754-8.

2. Elkan R, Kendrick D, Dewey M, Hewitt M, Robinson J, Blair M, et al. Effectiveness of home based support for older people: systematic review and meta-analysis. BMJ 2001;323(7315):719-25.

3. Stuck AE, Egger M, Hammer A, Minder CE, Beck JC. Home visits to prevent nursing home admission and functional decline in elderly people: systematic review and meta-regression analysis. JAMA 2002;287(8):1022-8.

4. Meinck M, Lubke N, Lauterberg J, Robra BP. [Preventive home visits to the elderly: systematic review of available evidence]. Gesundheitswesen 2004;66(11): 732-8.

5. Hall N. Randomized trial of a health promotion program for frail elders. Canadian Journal on Aging/La Revue Canadienne du vieillissement 1992;11(1):72-91.

6. Leveille SG, Wagner EH, Davis C, Grothaus L, Wallace J, LoGerfo M, et al. Preventing disability and managing chronic illness in frail older adults: a randomized trial of a community-based partnership with primary care.[comment]. J Am Geriatr Soc 1998;46(10):1191-8.

7. Pathy MS, Bayer A, Harding K, Dibble A. Randomised trial of case finding and surveillance of elderly people at home. Lancet 1992;340(8824):890-3.

8. Robichaud L, Hébert R, Roy PM, Roy C. A preventive program for community-dwelling elderly at risk of functional decline: a pilot study. Arch Gerontol and Geriatr 2000;30:73-84.

9. Nikolaus T, Bach M. Preventing falls in community-dwelling frail older people using a home intervention team (HIT): results from the randomized Falls-HIT trial. J Am Geriatr Soc 2003;51(3):300-5.

10. van Haastregt JC, Diederiks JP, van Rossum E, de Witte LP, Voorhoeve PM, Crebolder HF. Effects of a programme of multifactorial home visits on falls and mobility impairments in elderly people at risk: randomised controlled trial.[comment]. BMJ 2000;321(7267):994-8.

11. Dalby DM, Sellors JW, Fraser FD, Fraser C, van Ineveld C, Howard M. Effect of preventive home visits by a nurse on the outcomes of frail elderly people in the community: a randomized controlled trial. Canadian Medical Association Journal / Journal de l'Association Medicale Canadienne 2000;162(4):497-500.

12. Stuck AE, Minder CE, Peter Wuest I, Gillmann G, Egli C, Kesselring A, et al. A randomized trial of in-home visits for disability prevention in community-dwelling older people at low and high risk for nursing home admission. Archives of Internal Medicine 2000;160(7):97786.

13. van Rossum E, Frederiks CM, Philipsen H, Portengen K, Wiskerke J, Knipschild P. Effects of preventive home visits to elderly people. BMJ 1993;307(6895):27-32.

14. Nicolaides-Bouman A, van Rossum E, Kempen GI, Knipschild P. Effects of home visits by home nurses to elderly people with health problems: design of a randomised clinical trial in the Netherlands [ISRCTN92017183]. BMC Health Serv Res 2004;4(1):35.

15. van Haastregt JC. Preventing falls and mobility impairments in elderly people living in the community. PhD Thesis Maastricht University, 2002.

16. Hebert R, Robichaud L, Roy PM, Bravo G, Voyer L. Efficacy of a nurse-led multidimensional preventive programme for older people at risk of functional decline. A randomized controlled trial. Age and Ageing 2001;30(2):147-53.

17. Newbury JW, Marley JE, Beilby JJ. A randomised controlled trial of the outcome of health assessment of people aged 75 years and over. Medical Journal of Australia 2001;175(2):1047.

18. Vass M, Avlund K, Lauridsen J, Hendriksen C. Feasible Model for prevention of Functional Decline in Older People: Municipality-Randomized, Controlled trial. J Am Geriatr Soc 2005;53:563-568. 


\section{Chapter 3}

19. Gill T, Baker DI, Gottschalk M, Gahbauer E, Charpentier P, de Regt P, et al. A prehabilitation program for physically frail community-living older persons. Arch Phys Med Rehabil 2003;84(3):394-404.

20. Yamada Y, Ikegami N. Preventive home visits for community-dwelling frail elderly people based on Minimum Data Set-Home Care: Randomized controlled trial. Geriatrics and Gerontology International 2003;3(4):236-242.

21. Vass M, Avlund K, Kvist K, Hendriksen C, Andersen CK, Keiding N. Structured home visits to older people. Are they only of benefit for women? A randomised controlled trial. Scandinavian Journal of Primary Health Care 2004;22(2):106-11.

22. Oakley A, Strange V, Bonell C, Allen E, Stephenson J. Process evaluation in randomised controlled trials of complex interventions. BMJ 2006;332(7538):413-6.

23. Yura $\mathrm{H}$, Walsh $\mathrm{M}$. The nursing proces: assessing, planning, implementing, evaluating. 5th edition ed. Connecticut/San Mateo, California: Appletton-Century-Crofts, 1988.

24. de Groen B. Disease management: The American Dream? Soesterberg: Stichting Zorgverlening Het Zonnehuis, 2001.

25. Richardson J. The Easy-Care assessment system and its appropriateness for older people. Nurs Older People 2001;13(7):17-9.

26. Carpenito L. Handbook of Nursing Diagnosis. 4th ed. Philadelphia: J.B. Lippincott Company, 1993.

27. ICPC [http://www.hag.unimaas.nl/rnh/icpc.html].

28. van Rossum E. Effects of preventive home visits to the elderly. PhD Thesis Maastricht University, 1993.

29. Alessi CA, Stuck AE, Aronow HU, Yuhas KE, Bula CJ, Madison R, et al. The process of care in preventive in-home comprehensive geriatric assessment. J Am Geriatr Soc 1997;45(9):1044-50.

30. van Haastregt JC, van Rossum E, Diederiks JP, de Witte LP, Voorhoeve PM, Crebolder HF. Process-evaluation of a home visit programme to prevent falls and mobility impairments among elderly people at risk. Patient Educ Couns 2002;47(4):301-9.

31. Stuck AE, Aronow HU, Steiner A, Alessi CA, Bula CJ, Gold MN, et al. A trial of annual inhome comprehensive geriatric assessments for elderly people living in the community.[comment]. N Engl J Med 1995;333(18):1184-9.

32. Gussekloo J, de Bont LE, von Faber M, Eekhof JA, de Laat JA, Hulshof JH, et al. Auditory rehabilitation of older people from the general population--the Leiden 85-plus study. Br J Gen Pract 2003;53(492):536-40.

33. Luker K. Elderly women's opinions about the benefits of health visitor visits. Nursing Times 1981;77(12):suppl 9: 33-5. 


\title{
4
}

\section{Effects of a home visiting program for older people with poor health status: a randomized clinical trial in the Netherlands}

\author{
Ans Bouman ${ }^{1}$, Erik van Rossum ${ }^{2}$, Ton Ambergen ${ }^{3}$, \\ Gertrudis Kempen ${ }^{2}$ and Paul Knipschild ${ }^{4}$
}

J Am Geriatr Soc 2008, 56(3):397-404

1 Department of Epidemiology, Maastricht University, Maastricht, the Netherlands

2 Department of Health Care Studies, Maastricht University, Maastricht, the Netherlands

3 Department of Methodology and Statistics, Maastricht University, Maastricht, the Netherlands

4 Department of General Practice, Maastricht University, Maastricht, the Netherlands 


\begin{abstract}
Objectives

To evaluate the effectiveness of a home visiting program on health-related measures in a population of older people with poor health status.
\end{abstract}

\title{
Design
}

Randomized, clinical trial.

\section{Setting}

Community-dwelling citizens in the Netherlands.

\section{Participants}

Three hundred thirty people aged 70-84 randomly assigned to an intervention group $(\mathrm{n}=160)$ or a control group $(\mathrm{n}=170)$.

\section{Intervention}

Eight home visits, lasting 1 hour or more, with telephone follow-up, over an 18month period, conducted by experienced home nurses under supervision of a public health nurse; key elements of the (systematic) visits were assessment of health problems and risks, advice, and referral to professional and community services.

\section{Main outcome measures}

Self-rated health, functional status, quality of life, and changes in self-reported problems.

\section{Results}

No differences were found between the intervention and control group in these and other outcome measures at the end of the intervention period (18 months).

\section{Conclusion}

The home visiting program did not appear to have any effect on the health status of older people with poor health and are probably not beneficial for such persons. 


\section{Introduction}

With the growth in the ageing population, home visiting programs have been developed aimed at improving the health and independent functioning of older people and subsequently reducing outpatient care and hospital and nursing home admissions. In several countries, including Denmark, Australia, and Japan, home visiting programs are part of the national policy. The United Kingdom withdrew this policy in 2004. There is still an ongoing public health debate as to whether home visits should be incorporated into regular care for older people. The findings of trials on the effects of home visits have been inconsistent. $^{1-4}$

Although home visits for a high-risk population seems to be a promising approach, the results are mixed; seven controlled studies showed positive effects, ${ }^{5-11}$ but six other trials did not. ${ }^{12-17}$ A trial in the Netherlands $(n=580)$ showed that preventive home visits do not seem to be useful for the general population of older people, although a post hoc subgroup analysis indicated that the visits seemed to be effective for those with poor perceived health status at baseline. ${ }^{18}$ It was therefore decided to test this finding in a new trial, focusing entirely on older people in poor health. Details of the trial design have been published elsewhere. ${ }^{19}$ This article is a report on the effectiveness of the program on health-related measures. The effects on care utilization and a costbenefit analysis will be reported upon completion.

\section{Methods}

\section{Participants}

Eligibility of participants was determined through a questionnaire mailed in November 2002 to nearly 5,000 people (aged 70-84) living at home in the south of the Netherlands (Sittard and surrounding areas). Persons who reported their health status as moderate to good (a score of $\geq 6$ on a scale of 1-10), who already received home nursing care on a regular basis, or who were on a waiting list for admission to a nursing home or home for older persons were excluded. After the screening procedure, 160 persons were randomly allocated to the intervention group and 170 to the control group. ${ }^{19}$

\section{Sample size}

The sample size was calculated from data of a previous home visiting study in the Netherlands. ${ }^{18}$ Based on a 0.9 power to detect a difference (at the one-sided $5 \%$ level) of $20 \%$ or more between the study groups on self-rated health, with an assumed loss to follow up of 30\%, 150 participants were required in each group. 


\section{Randomization}

Before randomization, the 330 participants were divided into two groups: couples $(n=46)$ and those for whom this did not apply $(n=284)$. In this way, it was ensured that eligible persons who lived together were always allocated to the same study group. The 23 couples were distributed over three strata on the basis of their (summed) score on functional status. The other 284 participants were stratified into eight strata based on three prognostic factors: functional status, health change, and prior contact with a medical specialist. After stratification, the random allocation sequence was conducted using a computer generated randomization list with a block length of $4 .{ }^{19}$

\section{Intervention}

The program consisted of eight visits over an 18-month period. Participants in the intervention group received a visit approximately every 2 months, always from the same nurse. The visits lasted between 60 and 90 minutes. To increase adherence, the nurses contacted the elderly people by telephone 1 to 4 weeks after each visit. Three experienced home nurses (auxiliary community nurses) from a local home care organization were appointed half time for the study, and they conducted the visits under the supervision of a public health nurse (community nurse). The nurses followed a structured protocol to assess health problems and risks by interview. The nurses first recorded the problems as indicated by the participants. The EasyCare Questionnaire and additional checklists on a variety of topics were then used to detect further problems. ${ }^{20} \mathrm{As}$ part of this, the nurses could use diagnostic instruments for, for example, depressive complaints and mobility problems. No physical examinations were performed, because the home nurses were not qualified to do so. After the assessment, advice was given, or the elderly people were referred to professional and community services.

The participants' general practitioners (GPs) agreed to cooperate with the study and were kept informed at regular intervals. They received an overview of all treated problems for each participant in the intervention group, including the accompanying recommendations and results of the interventions. The GPs were asked for their comments or advice, and in this way they could become involved if they wanted to. In the case of urgent problems the nurses contacted the GPs according to standard communication procedures between the home care organizations and GPs.

The nurses were not part of a multidisciplinary team, but advice could be obtained from specialists within the home care organization (e.g. a dietician, a diabetes specialist and an occupational therapist). A nurse geriatric specialist from the local hospital could also be consulted to discuss cases. Further details of the program can be found in the design article. ${ }^{19}$ The home visits took place between February 2003 and October 2004. The control group received usual care; participants could use or apply for all available care within the Dutch health care system. 


\section{Outcome measures}

It was expected that the visits would improve the health status of the participants from baseline to 18 months (end of the intervention period). The primary health-related outcome measures are: self-rated health, functional status, activities of daily living (ADLs), instrumental activities of daily living (IADLs), ${ }^{21}$ quality of life, two subscales from the Medical Outcomes Study 20item Short Form Survey on mental health and social functioning, one item from RAND-36 on health change related to the start of the study, and changes in three self-reported problems, the first being the main problem. These were measured using mailed questionnaires at 12, 18, and 24 months (the last being 6 months after the end of the intervention). The screening questionnaire also provided baseline measurement for the primary outcomes. In addition, secondary outcome measures were gathered on health complaints, ${ }^{22}$ depressive complaints, ${ }^{23}$ cognitive status, ${ }^{24}$ mastery, ${ }^{25}$ social support, ${ }^{26}$ loneliness, ${ }^{27}$ and medication. These were measured after 18 months through individual interviews conducted by six trained, independent interviewers, blinded to group allocation. The municipality supplied mortality data over the study period.

\section{Statistical analyses}

The analyses were conducted according to the intention-to-treat principle. The primary outcomes self-rated health, functional status, and quality of life (mental health) were analyzed using a linear mixed-effects regression model within SPSS, version 13.0 (SPSS, Inc., Chicago, IL) at 12, 18, and 24 months. An unstructured covariance structure was used, and fixed effects were examined for the interaction between group and time. The outcomes quality of life (social functioning and health change) and changes in self-reported problems were analyzed using a two-level (time points and participants) proportional odds model within the STATA program GLAMM. ${ }^{28}$ To test for differences in effects of the secondary outcomes at 18 months, a general linear model was used (except for medication, for which a negative binomial regression model was used). All analyses were adjusted for possible differences in baseline values and background characteristics. Two-sided significance tests were used. Mean and standard deviations, adjusted mean differences between the study groups, odds ratios, and incidence-rate ratios, including 95\% confidence intervals (CIs) and P-values, are presented.

In addition, per-protocol analyses were conducted. For these analyses, it was required that participants had received all visits, adhered to $70 \%$ or more of all interventions, and had available data on all primary outcomes. Subgroup analyses on primary outcomes were performed for the following baseline variables: functional status, good versus bad (ADL-scores 11-19 and 20-44 respectively); health change, same or better versus worse; living situation, alone versus together; and mastery, average or much versus little. 
Ethical approval for the study was obtained from the Medical Ethical Committee of Maastricht University/Academic Hospital Maastricht. Information for patients about the aim of the study, procedures, protection of privacy, and covered insurance (as required by law), was included with the questionnaire. Return of a completed and signed informed consent form (included) was taken as consent to participate.

\section{Results}

\section{Participants}

Table 1 gives baseline characteristics of the 330 participants. The mean age was 76 years \pm 3.8 . Approximately half of this population had at least one ADLdependency, two IADL-dependencies, or both. Nearly half of the population indicated that their health had deteriorated in the 3 months before the screening. Approximately $40 \%$ of the participants in both groups received home care, which included home help and personal care, because persons receiving home nursing care had been excluded. The characteristics are comparable for both groups.

The program was largely implemented according to plan. Seventy-eight percent of participants $(\mathrm{n}=124)$ completed the entire program of eight visits. In total, 151 participants $(95 \%)$ received visits, on average seven visits per person with six follow-up telephone calls. In $91 \%$ of all visits, one or more problems were treated: on average 10 problems per person with 11 interventions $(38 \%$ referrals, $45 \%$ advice, and $17 \%$ information). The overall adherence rate with referrals and advice was $61 \%$. The average time spent on the visits in-home was 65 minutes \pm 11.8 . The program was feasible, and the participants and nurses appreciated it. More details of the process evaluation are reported elsewhere. ${ }^{29}$ Primary outcome measures were available for 293 persons: 139 in the intervention group (87\%) and 154 in the control group (91\%). A flow diagram of the participants is shown in Figure 1. 
Table 1 Baseline characteristics of the participants

\begin{tabular}{|c|c|c|c|c|}
\hline \multirow{2}{*}{$\begin{array}{l}\text { Characteristic } \\
\text { Age, mean } \pm \text { standard deviation }\end{array}$} & \multicolumn{2}{|c|}{$\begin{array}{l}\text { Intervention group } \\
\qquad(n=160)\end{array}$} & \multicolumn{2}{|c|}{$\begin{array}{l}\text { Control group } \\
\qquad(n=170)\end{array}$} \\
\hline & 75.8 & $(3.7)$ & 75.6 & (3.9) \\
\hline $\begin{array}{l}\text { Sex, } n(\%) \\
\text { male } \\
\text { female }\end{array}$ & $\begin{array}{l}64 \\
96\end{array}$ & $\begin{array}{l}(40) \\
(60)\end{array}$ & $\begin{array}{r}68 \\
102\end{array}$ & $\begin{array}{l}(40) \\
(60)\end{array}$ \\
\hline $\begin{array}{l}\text { Living situation, } \mathrm{n}(\%) \\
\text { alone } \\
\text { together }\end{array}$ & $\begin{array}{r}53 \\
103\end{array}$ & $\begin{array}{l}(34) \\
(66)\end{array}$ & $\begin{array}{r}61 \\
108\end{array}$ & $\begin{array}{l}(36) \\
(64)\end{array}$ \\
\hline $\begin{array}{l}\text { Education, } \mathrm{n}(\%) \\
\text { primary school } \\
\text { lower to middle professional education } \\
\text { higher professional education }\end{array}$ & $\begin{array}{l}60 \\
81 \\
15\end{array}$ & $\begin{array}{l}(39) \\
(52) \\
(10)\end{array}$ & $\begin{array}{l}65 \\
92 \\
11\end{array}$ & $\begin{array}{r}(39) \\
(55) \\
(6)\end{array}$ \\
\hline $\begin{array}{l}\text { Self-rated health*, n (\%) } \\
\quad 1-4 \\
5\end{array}$ & $\begin{array}{l}62 \\
98\end{array}$ & $\begin{array}{l}(39) \\
(61)\end{array}$ & $\begin{array}{r}67 \\
103\end{array}$ & $\begin{array}{l}(39) \\
(61)\end{array}$ \\
\hline $\begin{array}{cl}\text { Functional status }^{\dagger}, \mathrm{n}(\%) \\
\text { ADL-dependencies } & 0 \\
& 1-11 \\
\text { IADL-dependencies } & 0-1 \\
& 2-7 \\
\text { Total number } & 0-2 \\
& 3-18\end{array}$ & $\begin{array}{l}73 \\
86 \\
76 \\
79 \\
83 \\
75\end{array}$ & $\begin{array}{l}(46) \\
(54) \\
(49) \\
(51) \\
(53) \\
(47)\end{array}$ & $\begin{array}{l}81 \\
89 \\
83 \\
82 \\
92 \\
76\end{array}$ & $\begin{array}{l}(48) \\
(52) \\
(50) \\
(50) \\
(55) \\
(45)\end{array}$ \\
\hline $\begin{array}{l}\text { Health change in previous } 3 \text { months, n (\%) } \\
\text { same or better } \\
\text { worse }\end{array}$ & $\begin{array}{l}85 \\
75\end{array}$ & $\begin{array}{l}(53) \\
(47)\end{array}$ & $\begin{array}{l}82 \\
88\end{array}$ & $\begin{array}{l}(48) \\
(52)\end{array}$ \\
\hline $\begin{array}{l}\text { Health affects social participation, } \mathrm{n}(\%) \\
\text { often } \\
\text { sometimes } \\
\text { never }\end{array}$ & $\begin{array}{l}82 \\
49 \\
24\end{array}$ & $\begin{array}{l}(53) \\
(32) \\
(16)\end{array}$ & $\begin{array}{l}87 \\
50 \\
33\end{array}$ & $\begin{array}{l}(51) \\
(29) \\
(19)\end{array}$ \\
\hline $\begin{array}{l}\text { Mastery, } \mathrm{n}(\%) \\
\text { average or much influence on health } \\
\text { little influence on health }\end{array}$ & $\begin{array}{r}59 \\
100\end{array}$ & $\begin{array}{l}(37) \\
(63)\end{array}$ & $\begin{array}{l}79 \\
91\end{array}$ & $\begin{array}{l}(46) \\
(54)\end{array}$ \\
\hline $\begin{array}{l}\text { Contacted general practitioner in previous } 3 \\
\text { months, } \mathrm{n}(\%)\end{array}$ & 140 & (88) & 150 & $(88)$ \\
\hline $\begin{array}{l}\text { Contacted medical specialist in previous } 3 \\
\text { months, } \mathrm{n}(\%)\end{array}$ & 108 & $(69)$ & 117 & $(70)$ \\
\hline $\begin{array}{l}\text { Hospital admission in previous } 3 \text { months, } \\
\mathrm{n}(\%)\end{array}$ & 21 & $(13)$ & 24 & $(14)$ \\
\hline Use of home care, $\mathrm{n}(\%)$ & 64 & $(40)$ & 61 & $(37)$ \\
\hline $\begin{array}{l}\text { Note: This table (with the exception of Mast } \\
\text { * Indicated by a score on a scale ranging fr } \\
\text { health status (score <6) were included. } \\
\text { Refers to } 11 \text { activities of daily living (ADL } \\
\text { daily living (IADLs); dependencies indicat } \\
\text { older people are dependent on others to }\end{array}$ & $\begin{array}{l}10 \text { points. } \\
\text { seven ins } \\
\text { umber of } \\
n \text { the acti }\end{array}$ & $\begin{array}{l}\text { publishe } \\
\text { Particip }\end{array}$ & $\begin{array}{l}\text { sewhere } \\
\text { with po } \\
\text { tivities } \\
\text { which }\end{array}$ & \\
\hline
\end{tabular}




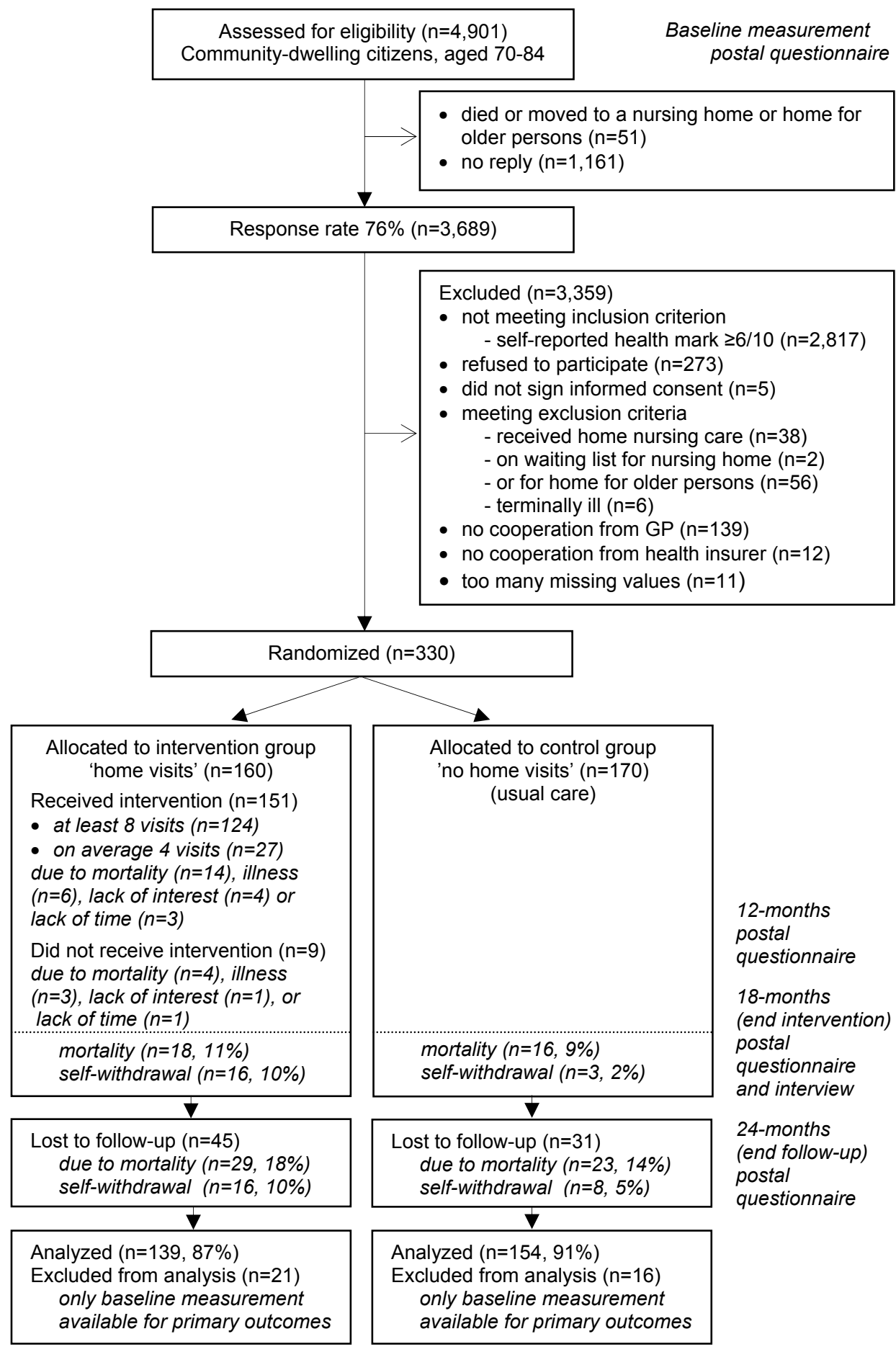

\section{Figure 1 Flow diagram of the participants}




\section{Outcome measures}

\section{Primary outcomes}

The mortality rates of the groups were not substantially different at the end of the intervention period; 18 intervention participants (11\%) and 16 control participants $(9 \%)$ died. The response rate for the questionnaires at 12,18 , and 24 months was approximately 95\%. Only the baseline measurement ( 0 months) was available for 37 of the 330 participants (21 intervention, 16 control), and they were omitted from the analyses on primary outcome measures. Of the remaining 293 participants, 244 completed all questionnaires, 27 missed one questionnaire and 22 participants missed two questionnaires at 12, 18 or 24 months (mainly because of mortality and self-withdrawal). The values of the missing questionnaires were substituted using the valid scores from the nearest time point in the intervention period.

A summary of the results for all primary outcome measures is given in Table 2 . At the end of the intervention period (18 months), few differences could be detected between the groups. After 12 months, the intervention group had slightly better scores than the control group on self-rated health, functional status (ADL), and health change (RAND-36). None of the differences between the groups reached significance at the $5 \%$ level.

Per-protocol analyses were carried out for 169 persons (44 intervention and 125 control). Again, no statistical significant differences were found between the study groups. These analyses were based on a small number of participants in the intervention group, because the per-protocol criteria were strictly applied. If a compliance rate of $60 \%$ or more was used with all interventions (instead of $70 \%$ ), the number of included participants in the intervention group increased to 75. This did not change the results.

\section{Secondary outcomes}

Table 3 shows the results of the secondary outcomes, measured based on individual interviews after 18 months. The outcomes were available for 263 persons (128 intervention (80\%) and 135 control $(79 \%))$. The intervention group scored slightly better than the control group on the health complaints subscales. No statistically significant differences were found between the two groups for any of the measures. 


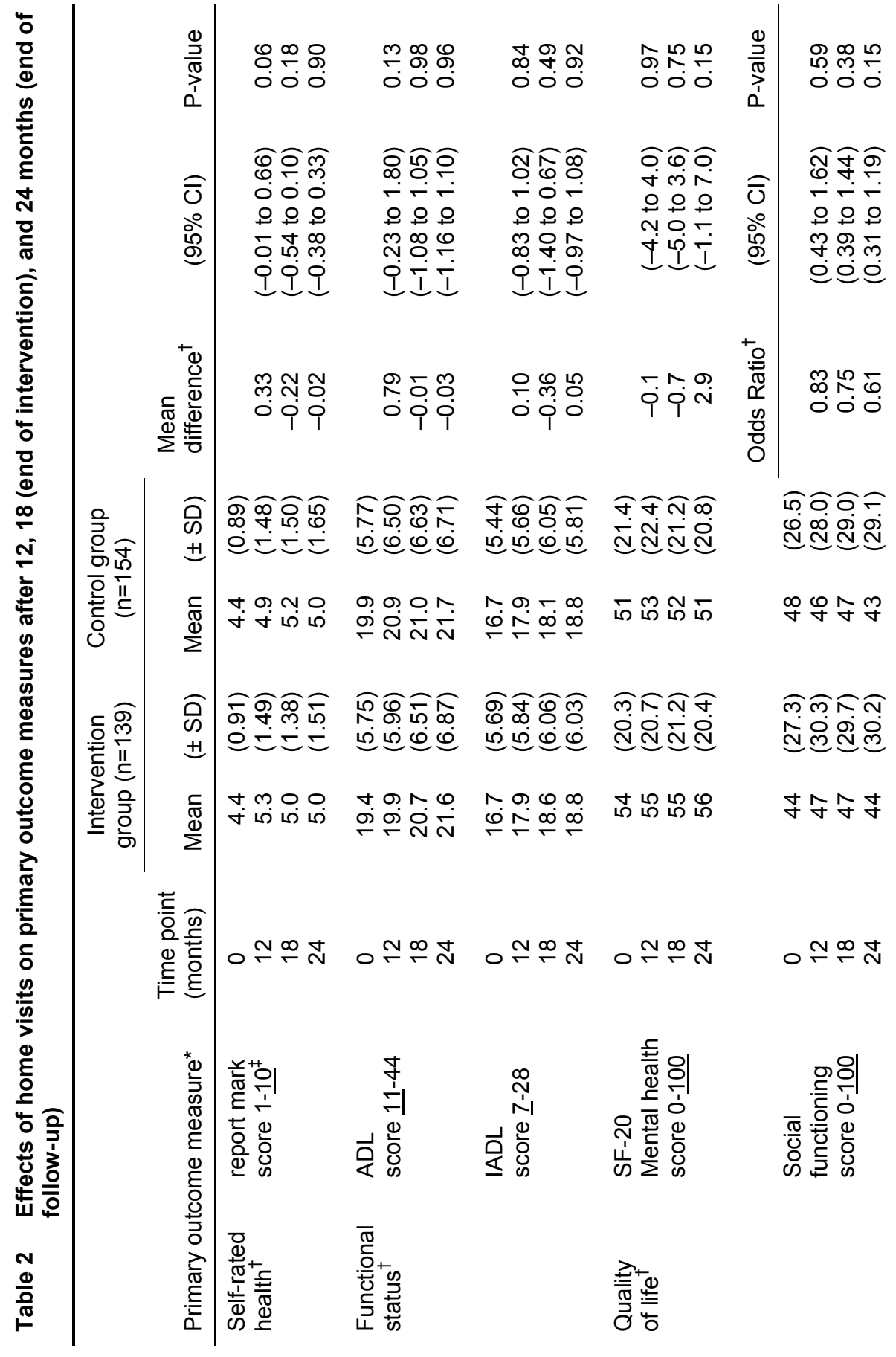




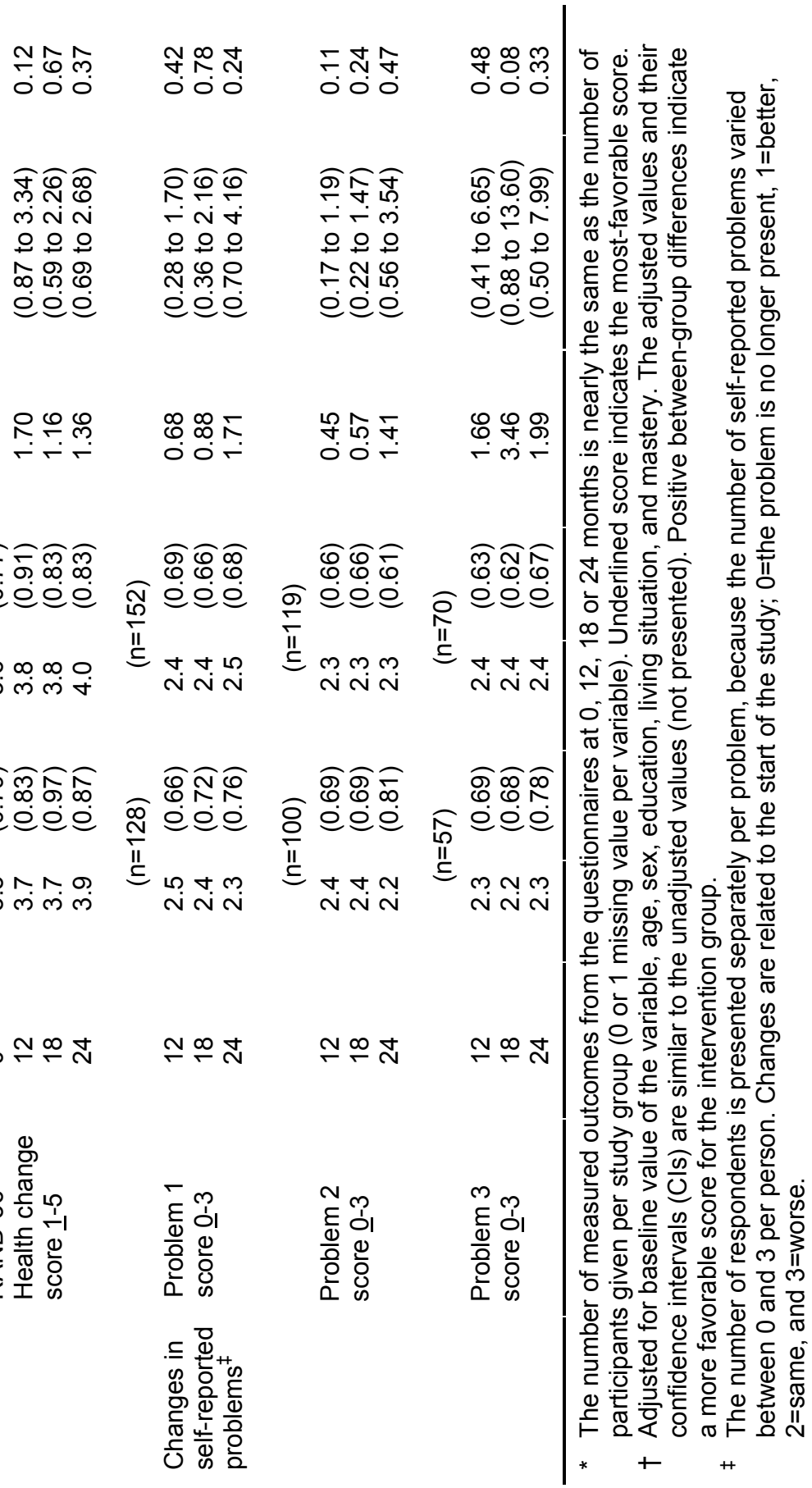




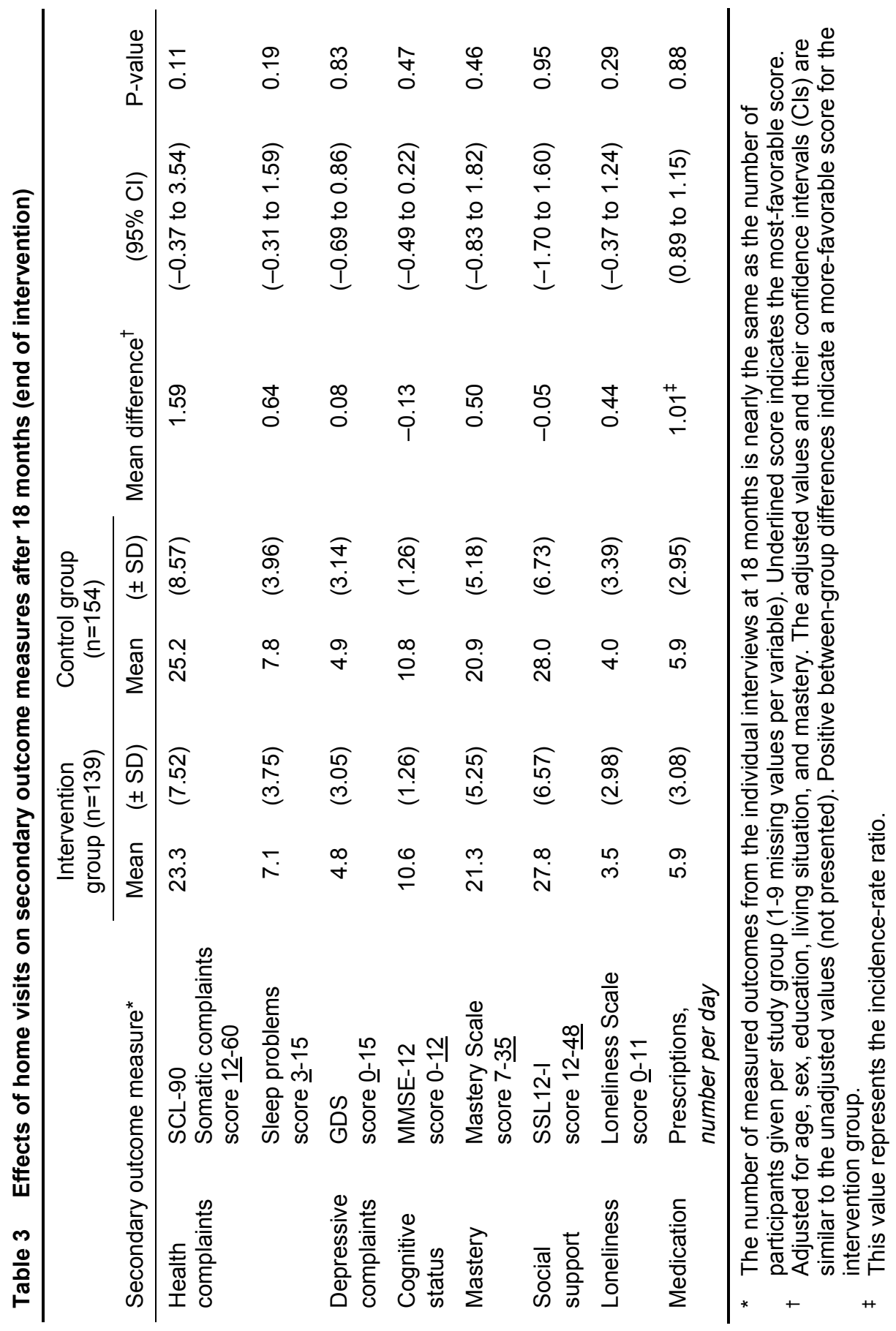




\section{Subgroup analyses}

At 18 months, the control group scored better than the intervention group on self-rated health in the subgroup 'good functional status' (0.49 points, 95\% $\mathrm{CI}=0.10-0.89)$, and in the subgroup 'same or better health' $(0.48$ points, $95 \%$ $\mathrm{CI}=0.08-0.89$ ). No effect was found in favor of any of the intervention subgroups at the end of the intervention period.

\section{Discussion}

The home visiting program carried out by home nurses had no effect on the health status of older people in poor health even though the program was performed under near ideal circumstances. Seventy-eight percent of participants completed the entire program of eight visits. The program seemed to be targeted towards a relevant population of elderly people with health problems; in nearly all visits a broad range of problems came forward. The program was feasible and appreciated by both participants and nurses. ${ }^{29}$ There were no methodological drawbacks. Selection bias does not seem obvious. After stratification and randomization, both groups were comparable. In addition, whether the applied randomization procedure allocating persons who lived together to the same study group influenced outcomes was tested using various statistical methods and was found not to be the case. Also, the response rate of the follow-up measurements was high. Most measurement scales used are internationally accepted instruments. It is unlikely that the small differences in mortality influenced the results.

Several other factors may have affected the effectiveness of the program. First, adherence to the interventions was reasonable (overall adherence rate of $61 \%$ ) and higher than reported in other home visiting studies (46-49\%), but this could still be increased to optimize possible beneficial effects. ${ }^{29}$ (The nurses may have improved overall adherence rate in our study by reminding and supporting the participants by means of a telephone follow-up contact after each visit.) Also, many referrals were to the GP (39\%). Adherence to the referrals was registered, but adherence of the patients to the GP's advice was not. Neither was whether applications for services or aids were honored registered. This may have further diluted the effect. Second, a more-intensive program, including, for example, more-frequent visits to support the participants with elements of behavioural change, might have been more beneficial, and could have added to the minor effects noticeable at 12 months. Third, although there is still much uncertainty about which specific aspects of interventions are effective, it has been suggested that higher-risk older subjects should be offered programs emphasizing systematic care-coordination. ${ }^{30}$ In the current study a continuous yield of health problems came forward throughout the program regarding social and psychological functioning, circulatory and musculoskeletal problems, and vision and hearing impairments. To manage these problems, many referrals 
were made to various care providers. ${ }^{29}$ It is possible that a more systematically planned coordination of care may have been more effective. Fourth, the nurses were not part of a multidisciplinary team. A community care setting, in which resources such as consultations with geriatricians are not readily available, was opted for to conduct the visits. If the program had generated significant beneficial effects, the visits could have been easily implemented in usual care, although multidisciplinary assessment and follow-up may result in morepositive outcomes when targeting specific groups of frail elderly people. Fifth, nurses who are qualified at a lower professional level (home nurses instead of public health nurses) were used. It is possible that their educational level was insufficient to manage the complex problems of the elderly people. Sixth, the health status of the participants may have been too poor; multiple problems were reported, and medication intake was high (on average six prescriptions daily). A possible irreversibility of their functional decline pattern may have been reached. Finally, many participants had already contacted their GP $(88 \%)$ or medical specialist $(70 \%)$ in the 3 months before the screening. It is possible that the intervention program did not add enough to the already existing services in the Dutch health care system.

The results of this study are compatible with those of the largest metaanalysis of preventive home visits in older people in which the authors conclude that the visits have no effect on mortality, nursing home admissions or functional status decline. ${ }^{3}$ Subgroup analyses in this review suggest benefits for certain types of patients or interventions: when programs were based on multidimensional assessment, involved multiple follow-up home visits and targeted people with lower risk for death. With respect to the last, trials in the meta-analyses with lower control group annual mortality (rates between $3.4 \%$ and 5.8\%) showed a beneficial effect on functional status, but this effect was not evident for trials with higher control group annual mortality (rates between $6.1 \%$ and $10.7 \%$, based on analyses of tertile ranges). Because the current study targeted people with poor health, and who most likely do not have a lower risk for death (the control group annual mortality rate was $6.8 \%$ ), benefits in this study were therefore not be expected according to these subgroup analyses. Another recent, large, home visiting trial targeting an older population with multiple health problems in the United Kingdom also showed no reduction in mortality or benefits for quality of life. ${ }^{16}$

Results of two other randomized, controlled trials in the Netherlands investigating the effectiveness of a problem-based community intervention model and a systematic demand-led home visiting program, both targeting frail elderly people, are expected to be published soon. ${ }^{31}{ }^{32}$ Because their results are not available, it is not yet possible to synthesize the findings of the three Dutch home visiting studies.

Self-rated health scores were better for the control group than the intervention group in the subgroups 'good functional status' and 'same or better health at baseline' at the end of the intervention period. This was the only 
detected difference in the subgroup analyses, and because there was no apparent relationship with various other aspects of health status, it is likely that this finding occurred merely by chance.

In neither the intention-to-treat nor the per-protocol analyses was any effect found of the home visits on health status. This result may also apply to comparable health care settings in other Western countries. A definitive assessment of the merits of the visiting program also depends on the effects on care utilisation. The analyses on the use of health services have not yet been completed, but preliminary results on the number of admissions to hospitals, nursing homes and homes for older persons also show no differences between the two study groups. This supports our conclusion that the home visiting program is probably not beneficial for elderly people with poor health. The use of all health services will be reported in due time together with a cost-benefit analysis.

There is much heterogeneity in results across the reported home visiting studies. Many factors can play a role in the effectiveness of the interventions, including the target population, characteristics of the intervention (content, number, and period of home visits), the effectiveness of different professionals and volunteers, and adherence to the recommendations. The variation in these factors probably contributes to the heterogeneity in results. Further research is necessary to determine which strategies are most beneficial, including the effectiveness of more-intensive programs, of systematic care-coordination and management, and of using higher levels of nursing qualifications. Attention should also be given to the communication between nurses and GPs to successfully implement interventions, because the GP holds an important position within many health care systems.

\section{Acknowledgements}

We thank the home care organization 'Thuiszorg Westelijke Mijnstreek', the municipality of Sittard-Geleen-Born, GPs and associations for elderly people in the Sittard region, and the research assistants. The grant application signatories were EvR, PK, Jos Diederiks, Harry Crebolder, and Hans Fiolet. The project group members were AB, EvR, RK, PK, Harry Heykens, Jan Houwen, Yvonne Monse, Truus Custers, and Annemarie Spaninks.

\section{Author contributions}

$\mathrm{AB}$ was responsible for coordinating the trial, analyzing the data, drafting the manuscript, and the intellectual content of the manuscript; she is the corresponding author. ER obtained funding; designed, initiated, and supervised the study; and was responsible for the intellectual content of the manuscript. GK 
supervised the study and was responsible for the intellectual content of the manuscript. TA was responsible for the statistical analysis of the data and the intellectual content of the manuscript. PK obtained funding; designed, initiated, and supervised the study; and was responsible for the intellectual content of the manuscript. All authors saw and approved the final version of the manuscript. 


\section{References}

1. van Haastregt JC, Diederiks JP, van Rossum E, de Witte LP, Crebolder HF. Effects of preventive home visits to elderly people living in the community: systematic review. BMJ 2000;320(7237):754-8.

2. Elkan R, Kendrick D, Dewey M, Hewitt M, Robinson J, Blair M, et al. Effectiveness of home based support for older people: systematic review and meta-analysis. BMJ 2001;323(7315):719-25.

3. Stuck AE, Egger M, Hammer A, Minder CE, Beck JC. Home visits to prevent nursing home admission and functional decline in elderly people: systematic review and meta-regression analysis. JAMA 2002;287(8):1022-8.

4. Meinck M, Lubke N, Lauterberg J, Robra BP. [Preventive home visits to the elderly: systematic review of available evidence]. Gesundheitswesen 2004;66(11):732-8.

5. Hall N. Randomized trial of a health promotion program for frail elders. Canadian Journal on Aging/La Revue Canadienne du vieillissement 1992;11(1):72-91.

6. Pathy MS, Bayer A, Harding K, Dibble A. Randomized trial of case finding and surveillance of elderly people at home. Lancet 1992;340(8824):890-3.

7. Leveille SG, Wagner EH, Davis C, Grothaus L, Wallace J, LoGerfo M, et al. Preventing disability and managing chronic illness in frail older adults: a randomized trial of a community-based partnership with primary care.[comment]. J Am Geriatr Soc 1998;46(10):1191-8.

8. Robichaud L, Hébert R, Roy PM, Roy C. A preventive program for community-dwelling elderly at risk of functional decline: a pilot study. Arch Gerontol Geriatr 2000;30:73-84.

9. Nikolaus T, Bach M. Preventing falls in community-dwelling frail older people using a home intervention team (HIT): results from the randomized Falls-HIT trial. J Am Geriatr Soc 2003;51(3):300-5.

10. Yamada Y, Ikegami N. Preventive home visits for community-dwelling frail elderly people based on Minimum Data Set-Home Care: Randomized controlled trial. Geriatrics and Gerontology International 2003;3(4):236-242.

11. Markle-Reid M, Weir R, Browne G, Roberts J, Gafni A, Henderson S. Health promotion for frail older home care clients. J Adv Nurs 2006;54(3):381-95.

12. Dalby DM, Sellors JW, Fraser FD, Fraser C, van Ineveld C, Howard M. Effect of preventive home visits by a nurse on the outcomes of frail elderly people in the community: a randomized controlled trial. Canadian Medical Association Journal / Journal de l'Association Medicale Canadienne 2000;162(4):497-500.

13. Stuck AE, Minder CE, Peter Wuest I, Gillmann G, Egli C, Kesselring A, et al. A randomized trial of in-home visits for disability prevention in community-dwelling older people at low and high risk for nursing home admission. Archives of Internal Medicine 2000;160(7):97786.

14. van Haastregt JC, Diederiks JP, van Rossum E, de Witte LP, Voorhoeve PM, Crebolder HF. Effects of a program of multifactorial home visits on falls and mobility impairments in elderly people at risk: randomized controlled trial.[comment]. BMJ 2000;321(7267):994-8.

15. Hebert R, Robichaud L, Roy PM, Bravo G, Voyer L. Efficacy of a nurse-led multidimensional preventive program for older people at risk of functional decline. A randomized controlled trial. Age and Ageing 2001;30(2):147-53.

16. Fletcher AE, Price GM, Ng ES, Stirling SL, Bulpitt CJ, Breeze E, et al. Population-based multidimensional assessment of older people in UK general practice: a cluster-randomized factorial trial. Lancet 2004;364(9446):1667-77.

17. Vass M, Avlund K, Lauridsen J, Hendriksen C. Feasible Model for prevention of Functional Decline in Older People: Municipality-Randomized, Controlled trial. J Am Geriatr Soc 2005;53:563-568.

18 van Rossum E, Frederiks CM, Philipsen H, Portengen K, Wiskerke J, Knipschild P. Effects of preventive home visits to elderly people. BMJ 1993;307(6895):27-32. 
19. Nicolaides-Bouman A, van Rossum E, Kempen GI, Knipschild P. Effects of home visits by home nurses to elderly people with health problems: design of a randomised clinical trial in the Netherlands. BMC Health Serv Res 2004;4(1):35.

20. EASY-Care [http://www.bbr-online.com/easycare].

21. Kempen GI, Miedema I, Ormel J, Molenaar W. The assessment of disability with the Groningen Activity Restriction Scale. Conceptual framework and psychometric properties. Soc Sci Med 1996;43(11):1601-10.

22. Derogatis LR, Rickels K, Rock AF. The SCL- 90 and the MMPI: a step in the validation of a new self-report scale. Brit J Psych 1976;128:280-289.

23. Yesavage JA, Brink TL, Rose TL, Lum O, Huang V, Adey M, et al. Development and validation of a geriatric depression screening scale: a preliminary report. Journal of Psychiatric Research 1982;17(1):37-49.

24. Breakhus A, Laake K, Engedal K. The Mini-Mental State Examination: identifying the most efficient variables for detecting cognitive impairment in the elderly. J Am Geriatr Soc 1992;40:1139-45.

25. Pearlin LI, Schooler C. The structure of coping. J Health Soc Beh 1978;19(1):2-21.

26. Kempen GI, van Eijk LM. The psychometric properties of the SSL12-I, a short scale for measuring social support in the elderly. Social Indicators Research 1995;35:302-12.

27. de Jong Gierveld J, van Tilburg TG. Measuring personal experiences and emotions with questionnaires: a study of the properties of the Loneliness-scale in different research projects. In: de Jong Gierveld \& van der Zouwen, editor. De vragenlijst in sociaal onderzoek: Een confrontatie van onderzoekspraktijk en -methodiek. Deventer: van Loghum Slaterus, 1987: 67-83.

28. Rabe-Hesketh S, Pickles A, Skrondal S. GLLAMM Manual. [http://www.bepress.com/ucbbiostat/paper160/:] J.C. Berkeley division of Biostatistics Working Paper Series. Working Paper 160, 2004.

29. Nicolaides-Bouman A, van Rossum E, Kempen GI, Habets H, Knipschild P. Home visiting program for older people with health problems: Process evaluation. J Adv Nurs 2007;58(5):425-35.

30. Bernabei R, Landi F, Gambassi G, Sgadari A, Zuccala G, Mor V, et al. Randomized trial of impact of model of integrated care and case management for older people living in the community. BMJ Clin res ed 1998;316(7141):1348-51.

31. Melis RJ, van Eijken MI, Borm GF, Wensing M, Adang E, van de Lisdonk EH, et al. The design of the Dutch EASYcare study: a randomized controlled trial on the effectiveness of a problem-based community intervention model for frail elderly people [NCT00105378]. BMC Health Serv Res 2005;5:65.

32. van Hout HP, Nijpels G, van Marwijk HW, Jansen AP, Van't Veer PJ, Tybout W, et al. Design and pilot results of a single blind randomized controlled trial of systematic demandled home visits by nurses to frail elderly persons in primary care [ISRCTN05358495]. BMC Geriatr 2005;5:11. 


\title{
5
}

\section{Effects on health care use and associated cost of a home visiting program for older people with poor health status: a randomized clinical trial in the Netherlands}

\author{
Ans Bouman ${ }^{1}$, Erik van Rossum², Silvia Evers ${ }^{3}$, Ton Ambergen ${ }^{4}$, \\ Gertrudis Kempen $^{2}$ and Paul Knipschild ${ }^{5}$
}

J Gerontol A Biol Sci Med Sci 2008, 63:291-297

1 Department of Epidemiology, Maastricht University, Maastricht, the Netherlands

2 School for Public Health and Primary Care, Maastricht University, Maastricht, the Netherlands

3 Department of Health Organization Policy and Economics, Maastricht University, Maastricht, the Netherlands

4 Department of Methodology and Statistics, Maastricht University, Maastricht, the Netherlands

5 Department of General Practice, Maastricht University, Maastricht, the Netherlands 


\begin{abstract}
Background

Home visiting programs have been developed to improve the functional abilities of older people and subsequently to reduce the use of institutional care services. The results of trials have been inconsistent and their cost-effectiveness uncertain. Home visits for a high-risk population rather than the general population seems a promising approach. We therefore studied the effects of a home visiting program for older people with poor health. This article describes the effects on health care use and associated cost.
\end{abstract}

\title{
Methods
}

We conducted a randomized clinical trial among 330 community-living citizens, aged 70-84 years, in the Netherlands. Participants in the intervention group $(n=160)$ received eight home visits by a trained home nurse over an 18-month period; a multidimensional geriatric assessment of problems was included. The main outcomes are: admissions to hospital, nursing home, and home for older persons; contacts with medical specialists, general practitioners, and paramedics; and hours of home care help. The data on health care use were mostly obtained from computerized databases of various medical administration offices; the follow-up period was 24 months.

\section{Results}

Inpatient and outpatient health care use was similar for both groups, with the exception of a higher distribution of aids and in-home modifications in favor of the intervention group. No differences were found between the intervention and control group in health care cost.

\section{Conclusion}

The home visiting program did not appear to have any effect on the health care use of older people with poor health and had a low chance of being costeffective. We conclude that these visits are probably not beneficial for such persons within the health care setting in the Netherlands or comparable settings in other Western countries. 


\section{Introduction}

Home visiting programs have been developed to improve the health and independent functioning of older people and subsequently to reduce the use of institutional care services. The findings of trials on the effects of home visits have been inconsistent. ${ }^{1-4}$ There is an ongoing debate whether home visits should be incorporated into regular care for older people. Only a limited number of trials have also addressed cost aspects. ${ }^{5-10}$ If home visits lead to a reduction in health care use and cost, this is an important argument in favor of continuation of such interventions.

Home visits for a high-risk population seems a promising approach, but the results are mixed; eight controlled studies showed positive effects, ${ }^{6}{ }^{11-17}$ six other trials did not. ${ }^{78-22}$ An earlier trial in the Netherlands $(n=580)$ showed that preventive home visits do not seem to be useful for the general population of older people, although a post hoc subgroup analysis indicated that the visits seemed to be effective for those with a poor perceived health status. ${ }^{23} \mathrm{We}$ therefore decided to reinvestigate this finding, focusing entirely on older people with poor health. Details of the design of the current trial, process evaluation of the intervention, and effects on health status have been published elsewhere. ${ }^{24-26}$ The home visiting program did not show a significant effect on health status for instance, on the primary outcomes self-rated health, functional status or quality of life. Nevertheless, it is possible that the program might have generated sufficient cost offsets in reduced health expenditures that could yield significant health savings overall. This article describes the effects on health care use and associated cost. Additionally, a cost-effectiveness analysis was performed from a societal perspective.

\section{Methods}

\section{Participants}

Eligibility of participants was determined through a questionnaire, which was mailed in November 2002 to 4,901 people (70-84 years) living at home in a town in the south of the Netherlands (Sittard and surrounding areas). We excluded persons who valued their health status as moderate to good (self-rated health $[\mathrm{SRH}] \geq 6$, scale $1-10$ ); who already received home nursing care on a regular basis; or who were on a waiting list for admission to a nursing home or home for older persons. After the screening procedure, we randomly allocated 160 persons to the intervention and 170 to the control group. The sample size was calculated from data of a previous home visiting study in the Netherlands on the primary health-related measure $\mathrm{SRH},{ }^{6}{ }^{24}$ which represents an overall measure for functional health abilities, including physical, mental, and social functioning. ${ }^{23}$ Based on a 0.9 power to detect a difference (at the one-sided 
$5 \%$ level) of $20 \%$ or more between the study groups on SRH, with an assumed loss to follow-up of $30 \%, 150$ participants were required in each group.

\section{Intervention}

The program consisted of eight home visits, with telephone follow-up, over an 18-month period (February 2003 to October 2004). Participants in the intervention group were visited approximately every 2 months. Three trained home nurses (auxiliary community nurses) conducted the visits under supervision of a public health nurse (community nurse). Key elements of the (systematic) visits included a multidimensional geriatric assessment of problems and risks, advice, and referral to professional and community services. ${ }^{24}$ The control group received usual care; participants could use or apply for all available care.

\section{Health care use and cost}

Health care use relates to all professional health services and goods consumed during the intervention period and 6-month follow-up. These services include number of admissions and length of stay at the hospital, nursing home, and home for older persons; number of contacts with medical specialists, general practitioners (GPs), and paramedics; and hours of home care help. Goods consumed include medication, aids, and in-home modifications. Data on hospital admissions and contacts with medical specialists and GPs were also available for a 6-month period before the start of the intervention (baseline values). The volumes of the health care items were mostly obtained from computerized databases of various medical administration offices (see Table 1). The municipality supplied mortality data.

Almost all cost prices were obtained from Dutch guidelines; the baseline year was 2003 , or otherwise discounted at $4 \% .{ }^{28}{ }^{29}$ For medication and aids we used the cost supplied by the health insurance companies. The cost of in-home modifications was based on average prices per item. Health care costs are inpatient days spent at the hospital, nursing home, home for older persons (and day treatments in the hospital); outpatient visits to health care providers; professional home care; medication; aids; and in-home modifications. The cost of the intervention program is presented separately from the other health care cost and includes the nurses' salaries, their travel cost and the cost of their training activities.

\section{Cost-effectiveness analysis}

For the cost-effectiveness analysis we calculated the incremental cost and effectiveness of the home visiting program compared with usual care. 
Table 1 Health Care Items

\begin{tabular}{|c|c|c|c|}
\hline Category & Volume & Cost* $^{*}$ & Source of Data ${ }^{\dagger}$ \\
\hline \multicolumn{4}{|l|}{ Health care: inpatients } \\
\hline Hospital & $\begin{array}{l}\text { admission } \\
\text { day }\end{array}$ & $\overline{3} 37.00$ & $\begin{array}{l}\text { Local hospital } \\
\text { Health insurance organization }\end{array}$ \\
\hline Day treatment & day & 229.00 & Local hospital \\
\hline Nursing home & $\begin{array}{l}\text { admission } \\
\text { day }\end{array}$ & $\overline{2} 206.00$ & Office for public financed care \\
\hline Home for older persons & $\begin{array}{l}\text { admission } \\
\text { day }\end{array}$ & $\overline{85.00}$ & Office for public financed care \\
\hline \multicolumn{4}{|l|}{ Health care: outpatients } \\
\hline Medical specialist & consultation & 56.00 & $\begin{array}{l}\text { Local hospital } \\
\text { Individual interviews }\end{array}$ \\
\hline GP & $\begin{array}{l}\text { consultation } \\
\text { home visit } \\
\text { telephone }\end{array}$ & $\begin{array}{l}20.20 \\
40.40 \\
10.10\end{array}$ & GP office \\
\hline Paramedical therapy ${ }^{\S}$ & consultation & 22.75 & $\begin{array}{l}\text { Local hospital } \\
\text { Health insurance organization }\end{array}$ \\
\hline $\begin{array}{l}\text { Practical assistance at } \\
\text { home }\end{array}$ & hour & 21.70 & $\begin{array}{l}\text { Office for public financed care } \\
\text { Local home care organization" }\end{array}$ \\
\hline Personal care at home & hour & 34.10 & $\begin{array}{l}\text { Office for public financed care } \\
\text { Local home care organization" }\end{array}$ \\
\hline Community nurse visits & hour & 58.90 & $\begin{array}{l}\text { Office for public financed care } \\
\text { Local home care organization" }\end{array}$ \\
\hline $\begin{array}{l}\text { Medication (prescription } \\
\text { drug) }\end{array}$ & number ${ }^{\pi}$ & per drug $^{\#}$ & $\begin{array}{l}\text { Health insurance organization } \\
\text { Individual interviews }\end{array}$ \\
\hline Aids & number ${ }^{\pi}$ & $\begin{array}{l}\text { total }^{* *} \\
-\end{array}$ & $\begin{array}{l}\text { Health insurance organization } \\
\text { Individual interviews }\end{array}$ \\
\hline In-home modifications & number ${ }^{\pi}$ & per item & Individual interviews \\
\hline
\end{tabular}

* Prices $(2003 €)$ from the Dutch manual for cost-analysis in health care research, ${ }^{28}$ unless otherwise stated.

$\dagger$ Continuous registration over 24 months; data from individual interviews at 18 months. Missing data: general practitioners (GP) offices ( $n=9,3$ intervention and 6 control), health insurances ( $n=11,7$ intervention and 4 control), and individual interviews $(n=67,32$ intervention and 35 control), due to mortality $(n=34)$, self-withdrawal $(n=19)$, and illness $(n=14)$.

$\ddagger$ Complementary data for nonlocal hospital admissions $(20 / 291,7 \%)$ and specialist consultations $(321 / 2816,11 \%)$.

$\S$ Mainly physiotherapy.

II Complementary data for 2003; $41 / 330$ participants (12\%) were not registered at the local organization, of these 41,14 received home care according to self-reported data at baseline and these data could not be obtained.

I Number of prescriptions at 18 months/number of acquired aids and in-home modifications over 18 months (prestructured format of 12 and 11 items, respectively); data from individual interviews.

\# Cost per drug includes the pharmacist fee $(€ 6.45)$; data from health insurances.

** Total cost; data from health insurances. 
Incremental cost are defined as the mean difference between both groups in total cost over 24 months; incremental effectiveness is the mean difference in SRH at 24 months, adjusted for baseline values. ${ }^{26}$ The value of production lost to society due to illness-related absence from work was not assessed, because this is of no relevance in the targeted population.

\section{Statistical analyses}

The analyses were conducted according to the intention-to-treat principle. For hospital admissions, contacts with medical specialists, GPs, and paramedics, and hours of home care, we assessed differences between the intervention and control group, applying a generalized linear model for generalized estimating equations. This model allowed for analysis of repeated measurements (consumption at half-yearly periods) and negative binomial distributions for count data (due to skewed distributions). The remaining variables were analyzed either by a Cox regression analysis or a general linear model for negative binomial distributions. All analyses (SPSS, version 15.0; SPSS, Chicago, IL) were adjusted for possible differences in baseline values, if available, and baseline characteristics. Two-sided significance tests were used. Mean and standard deviations (SD), incidence-rate and hazard ratios (including $95 \%$ confidence intervals $[\mathrm{CI}]$ ), and $\mathrm{p}$-values, are presented.

To examine the uncertainty surrounding sample selection for both cost and effects, we conducted bootstrap simulations. ${ }^{30}$ Finally, we performed sensitivity analyses.

\section{Results}

\section{Participants}

In total, 330 people participated, 160 in the intervention and 170 in the control group. The mean age of the participants was 76 years $(\mathrm{SD}=3.7)$, with $40 \%$ men and $60 \%$ women. Baseline characteristics were comparable for both groups. ${ }^{24}$ Mortality showed no substantial differences between the groups after 24 months; 29 intervention participants (18\%) and 23 control participants (14\%) died. Seventy-eight percent of the intervention participants (124/160) received all eight visits and another $17 \%(27 / 160)$ on average four. The reasons for receiving only some of the visits were mortality, self-withdrawal or illness. ${ }^{25}$

\section{Health care use and cost}

Data on health care use were available for nearly all participants, including those who died during the 2-year study period. Data for 11/330 participants (3\%) could not be obtained from the health insurance companies; and for $9 / 330$ participants (3\%) from the GP offices (see also footnote to Table 1). 
Tables 2 and 3 summarize, respectively, the participants' inpatient and outpatient health care use. Baseline values are also presented, if available. A higher percentage of participants in the intervention group were admitted to the hospital, but the mean length of stay was slightly shorter. Approximately half of the participants who were admitted to the hospital over 24 months were admitted once (40/80 intervention and 34/71 control), approximately $30 \%$ twice (25/80 intervention and 21/71 control), and the remaining $20 \%$ three times or more (15/80 intervention and 16/71 control) (not tabulated). Overall, no statistically significant differences were found between the two groups for inpatient health care use.

The proportion of participants consulting different medical specialists at the outpatient department (18 specialties recorded in total) was similar in both groups, e.g. $40 \%$ of the participants contacted an ophthalmologist $(63 / 160$ intervention and $71 / 170$ control), $25 \%$ a cardiologist (42/160 and 40/170), and approximately $25 \%$ a neurologist (42/160 and 41/170). Few participants consulted a geriatrician (6/160 intervention and 15/170 control) (not tabulated). The mean number of consultations and visits from the GP was slightly higher in the intervention group compared to the control group; whereas the mean number of telephone contacts was lower (Table 3). Approximately the same percentage of persons in both groups was having professional home care; the mean number of hours was somewhat higher in the intervention group. Nearly everybody used medication, which we classified according to the Anatomical Therapeutic Chemical Classification System; ${ }^{31}$ e.g. $76 \%$ in the intervention and $67 \%$ in the control group used drugs for the cardiovascular system and over half for the nervous system (55\% and 57\%, respectively). Hardly any differences were found between the groups in any of the drug classes, in either the percentage of users or the mean numbers per drug class (not tabulated). None of the results on noninstitutional care demonstrated statistically significant differences between the groups.

More aids were acquired during the intervention period by participants in the intervention group compared to the control group (incidence-rate ratio 1.6, 95\% $\mathrm{CI}=1.2-2.0$ ); e.g. for mobility (rollators, $25 \%$ vs $18 \%$; scoot mobiles, $13 \%$ vs $8 \%$ ), for reading $(9 \%$ vs $4 \%$ ), and for getting dressed (5\% vs $1 \%$ ) (not tabulated). In-home modifications were also acquired more often by intervention participants compared to control participants (incidence-rate ratio $1.5,95 \% \mathrm{CI}=1.2-1.9)$, ranging from a heightened toilet seat ( $21 \%$ vs $16 \%$, grips for toilets (18\% vs $11 \%$ ) and for showers (30\% vs $14 \%)$, to alarm systems (5\% vs $2 \%)$ (not tabulated). 
In Table 4 the use of health care resources was valued in monetary units. The use of more aids and in-home modifications by the intervention group was not reflected by higher cost; the cost for aids was even lower. The cost of in-home modifications was calculated per item (11 items), and although the number was higher in the intervention group, the cost was counterbalanced by a higher number of expensive items in the control group (e.g. chair lift, $5 \%$ vs $4 \%$, and central heating, $3 \%$ vs $1 \%$ ) (not tabulated). The overall total cost per person, including the cost for the home visiting program, is $€ 450$ higher in the intervention group than in the control group. The overall differences are not statistically significant. The sensitivity analyses did not change the results (details on request).

\section{Cost-effectiveness}

For 37/330 participants only the baseline measurement of the primary outcomes was available; they were omitted from the analyses. In total there were four measurements (questionnaires) at baseline, 12, 18, and 24 months. Values were substituted on an individual basis from the nearest available value in the intervention period, if persons had one or two missing questionnaires (due to mortality, self-withdrawal, or illness) ${ }^{26}$ Data on the effect measure SRH was therefore available for 293 participants (139 intervention [87\%] and 154 control [91\%]). For those we calculated the total mean cost. This resulted in an incremental cost of $€ 1525$ (95\% CI $=-€ 2251$ to $€ 5299)$, or higher cost for the intervention group (not tabulated). The mean difference in SRH between both groups at 24 months $(\mathrm{n}=293)$ was -0.02 points $(95 \% \mathrm{CI}=-0.38$ to 0.33 , $\mathrm{p}=0.90) .{ }^{26}$ There appeared to be no difference in total cost and in scores on SRH between the intervention and the control groups. Bootstrap analysis confirmed these results and showed furthermore that there was only a $10 \%$ chance that the program was cost-effective (details on request). 


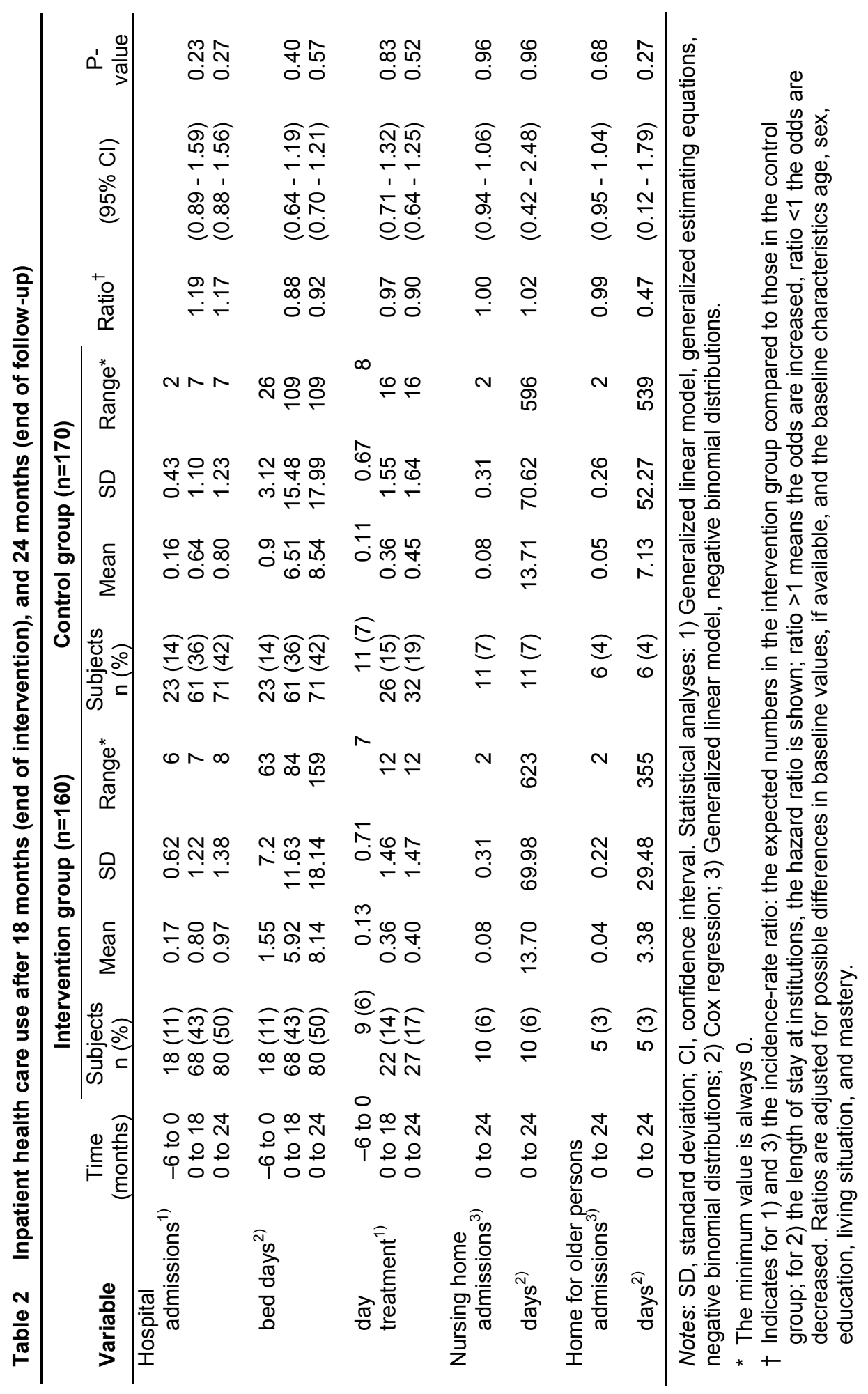




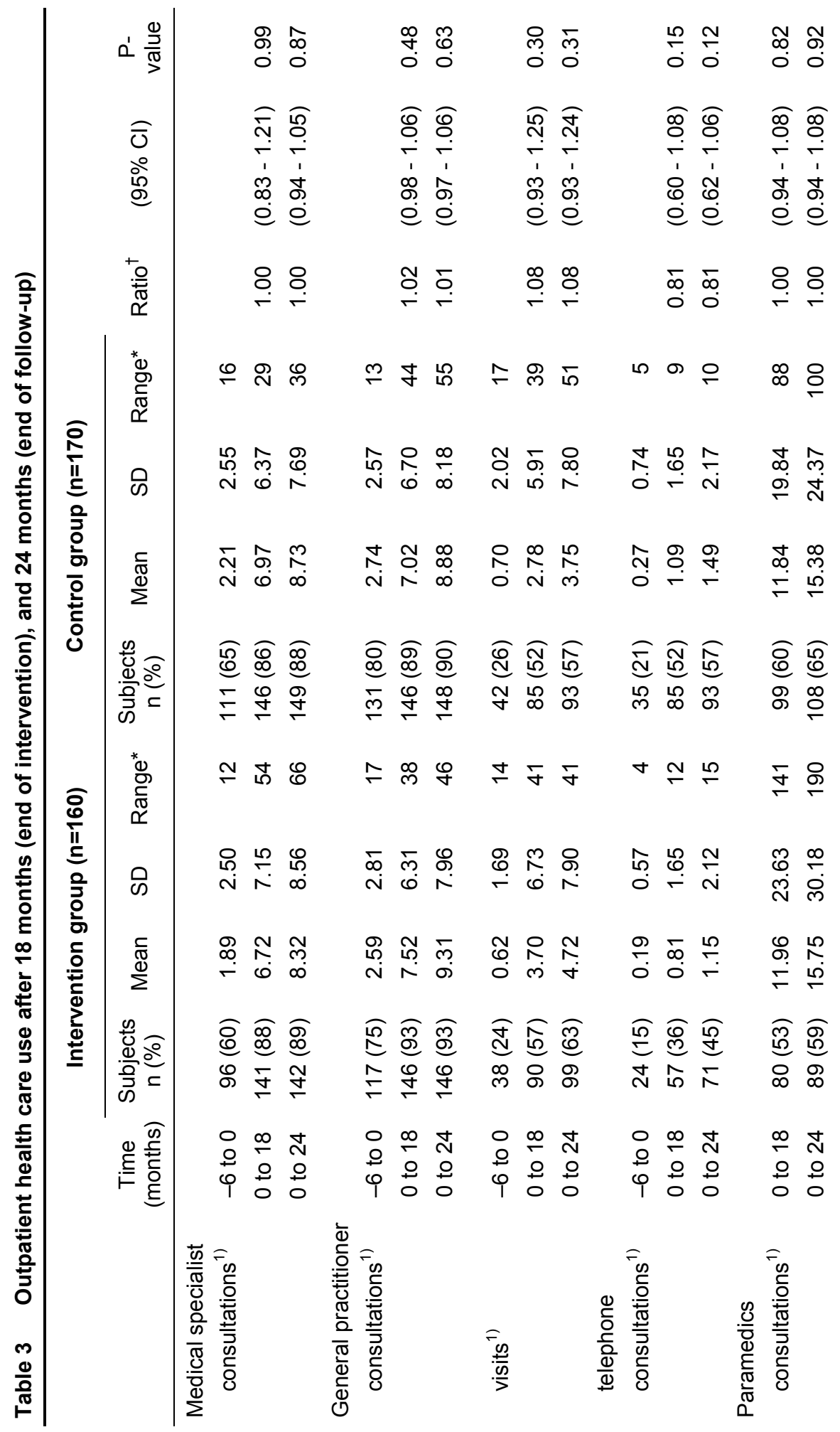




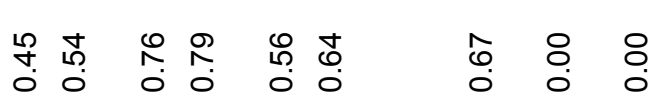

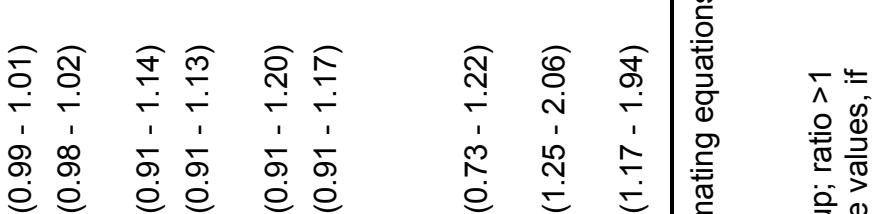

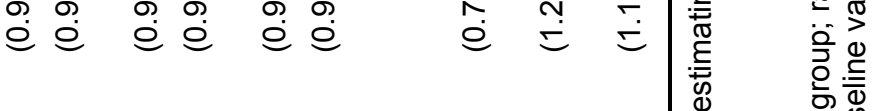

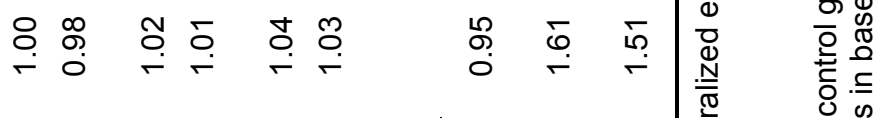

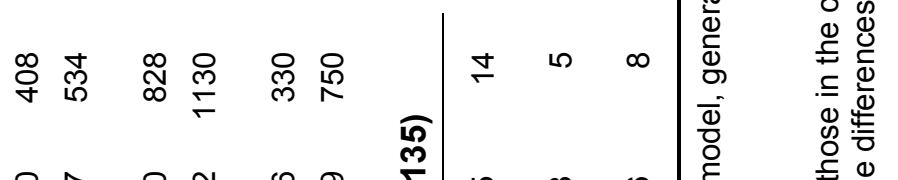

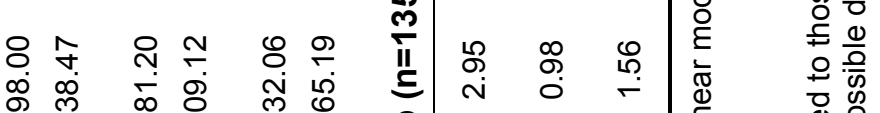

๑

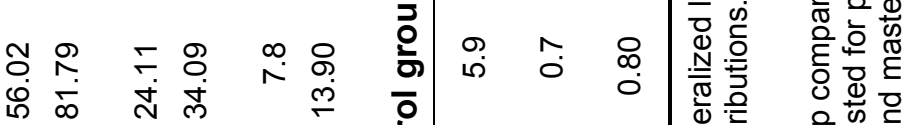

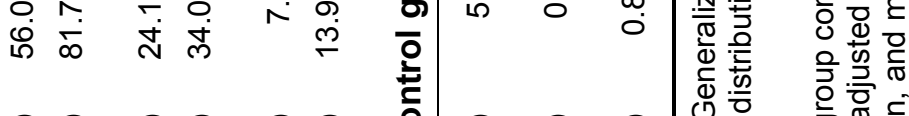

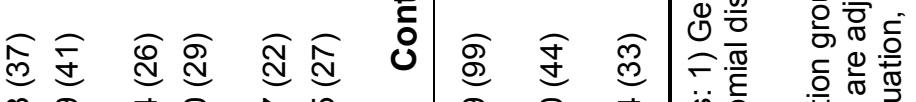

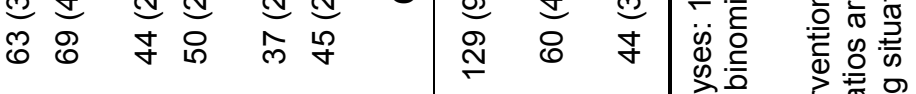

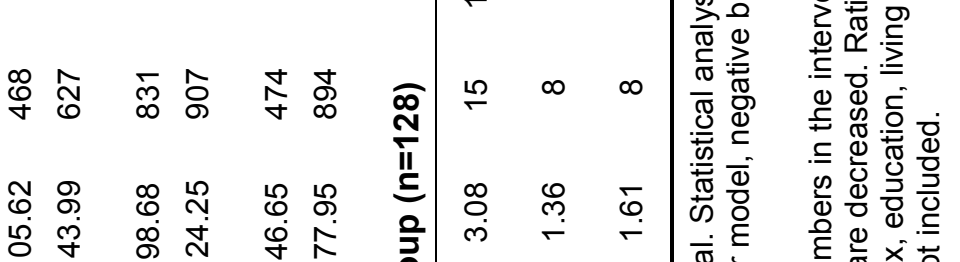

官

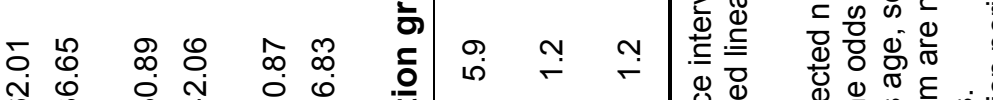

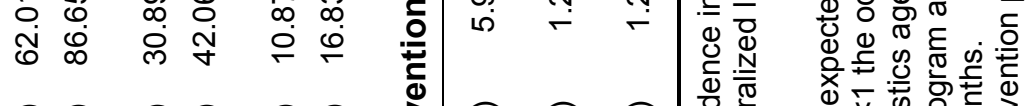

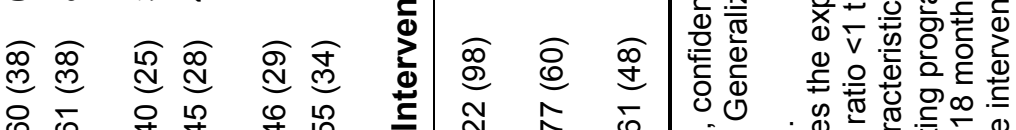
$8 \overline{0}$ \& $\quad$ \&

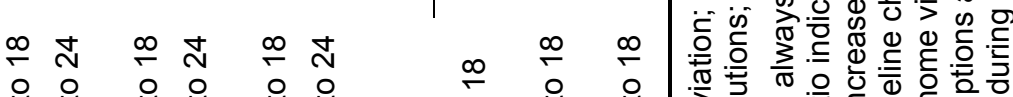

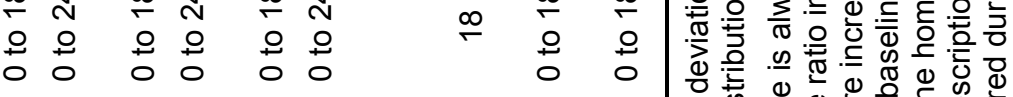

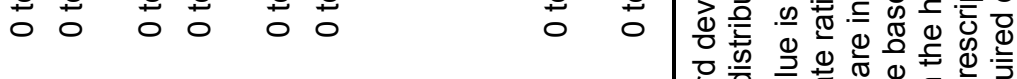

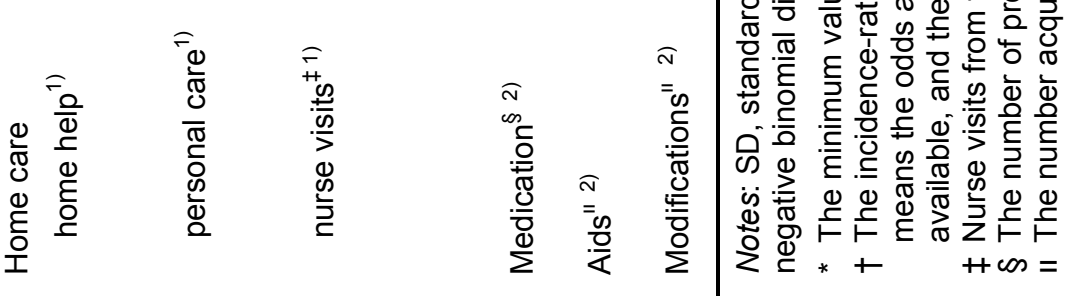




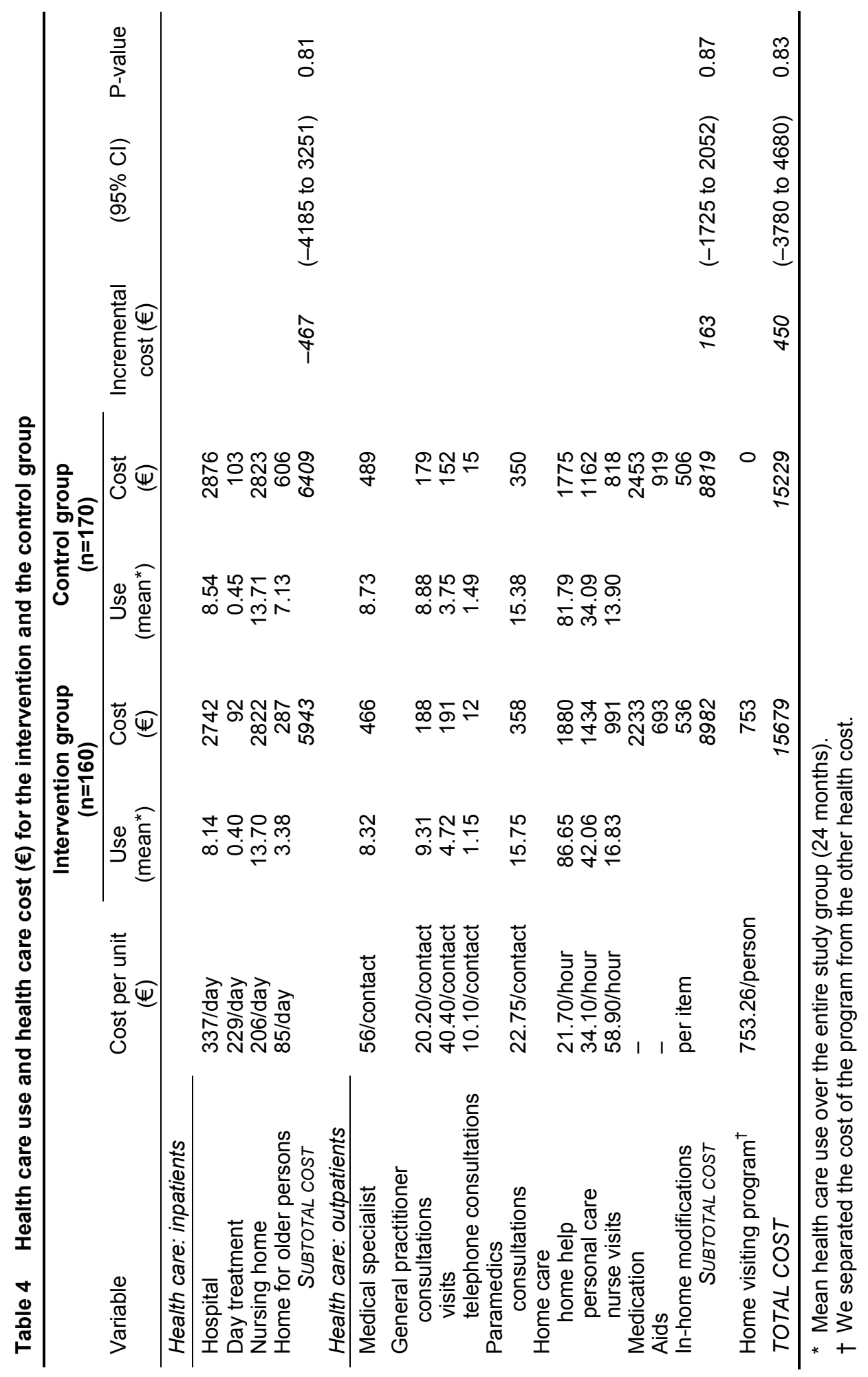




\section{Discussion}

Overall, we could not show a positive effect of the home visiting program conducted by home nurses on the health care use of older people with poor health. The results of the economic evaluation showed furthermore that the program had no effect on cost and had a low chance of being cost-effective. The home visiting program was performed nevertheless under near ideal circumstances. $^{25} 26$ The health care data that we collected were mostly from official registries and had a high degree of completeness. It is unlikely that the small differences in mortality influenced the results. Because there were no health effects, it is unlikely that cost past 24 months would be greatly reduced by the intervention.

The most important elements of the visits were to detect problems and risks, to give advice and to refer to other professional or community services. A continuous yield of health problems came forward, and to manage these problems many referrals were made to various care providers. ${ }^{25}$ Before the start of the program we expected an increase in outpatient health care use and subsequently a decrease in institutional care. On the basis of the referrals, on average four referrals per person during the intervention period $(n=144,650$ referrals), an increase was to be expected in GP contacts (39\% of referrals), aids and in-home modifications ( $15 \%$ of referrals), and home care (13\% of referrals). The compliance rate was also highest for those referrals (between $69 \%$ and $82 \%$ ). Referrals to medical specialists accounted for $8 \%$ of the referrals (compliance rate $65 \%$ ) and to physical therapists $4 \%$ (compliance rate $52 \%$ ). ${ }^{25}$ We did find an increase, although not statistically significant, in GP contacts in the intervention group compared with the control group and a higher number of hours in home care. More aids were acquired by the intervention group, and inhome modifications were done more frequently. It was not surprising, due to the lower number of referrals and lower compliance, that hardly any differences were found between the groups in the number of medical specialist and physical therapist contacts. The small increases in outpatient health care use for the intervention group did not, however, have any impacts on the use of institutional health care.

Several other factors may have affected the effectiveness of the program on health care use and cost. First, the nurses were not part of a multidisciplinary team. We opted for a community care setting, in which resources such as consultations with geriatricians and physicians are not readily available, to perform the visits. This limited, however, the medical component of the geriatric assessment and may have resulted in fewer and different referrals to various care providers. Second, other program characteristics, including for example more frequent visits ${ }^{3}$ or a more systematically planned coordination of care, ${ }^{32}$ might have added to the minor effects on health care use. Third, many participants used the health care system; around $90 \%$ contacted their GP or a 
medical specialist, and between $30 \%$ and $40 \%$ received home care. Although there were some shifts in health care use patterns, it cannot be ruled out that, in general, usual care is sufficient in this health care setting. Fourth, the study sample size calculations (as in most cost-effectiveness studies) were based on effectiveness and not on service use or cost measures, which have much higher coefficients of variation than $\mathrm{SRH}$, and so generally require larger sample sizes. Fifth, we did not include the cost of informal care, which could have been of relevance for the targeted population, e.g. paid and unpaid help from family and friends. Finally, for aids and in-home modifications it is recommended to use data from care providers rather than self-reported data, because they less underestimate volumes and cost. ${ }^{33}$ Cost of in-home modifications in this study might have been underestimated. The cost for aids, as supplied by the health insurance companies, was however lower than we expected for the intervention group (based on the collected 12 items in this study).

The results of the current study on health care use are compatible with those found by Stuck and colleagues and Dalby and colleagues ${ }^{718}$ Both trials also did not find effects on hospital or ambulatory care use among persons at risk of functional deterioration. The cost-effectiveness findings in this study are in agreement with those of Kronborg and colleagues, ${ }^{10}$ who also included an economic evaluation in their study on the effectiveness of home visits for older people. Their study also showed no significant differences in total cost or effectiveness. It is however difficult to compare this study with the one by Kronborg and colleagues, because they targeted the general population of home-living citizens, focusing mainly on nondisabled persons to prevent functional decline, and their visiting program consisted of, on average, 1.5 visits per participating person during the 3 -year program. From other trials addressing cost aspects, it still remains uncertain whether home visits are cost-effective. ${ }^{5-9}$

Beforehand we expected that the home visits would improve the health status of the participants and reduce institutional care. The current study could not, however, demonstrate this. We did not find effects of the home visits on health status or on health care use and associated cost. The additional aids and in-home modifications might have made life more comfortable in the intervention group, but this did not affect their health status. In conclusion, we think that the home visiting program including multidimensional geriatric assessment with advice and referral to professional and community services is probably not beneficial for older people with poor health within the health care setting in the Netherlands or comparable settings in other Western countries.

The post hoc subgroup comparison from an earlier Dutch study that indicated the visits to be effective for those with a poor perceived health status at baseline could not be confirmed by the results from this larger study. Post hoc subgroup analyses should be interpreted with caution. Further research is necessary to determine which strategies are most beneficial, including for instance the effectiveness of more intensive programs. 


\section{Acknowledgements}

We thank the home care organization 'Thuiszorg Westelijke Mijnstreek': Harry Heykens, Jan Houwen, and the nurses Ria Claessens, Ine Janssen, Hanneke de Jongh, and Yvonne Monse; the municipality of Sittard-Geleen; Maasland hospital; the Office for public financed care; the health insurance organizations, general practitioners, and the associations for elderly people in the Sittard region; research assistants Truus Custers, Annemarie Spaninks, and Marion Gijbels; and the interviewers.

\section{Author contributions}

$\mathrm{AB}$ was responsible for coordinating the trial, analyzing the data, and drafting the paper. ER obtained funding; designed, initiated and supervised the study. SE helped with the economic evaluation, and TA helped with the statistical analysis. GK supervised the study. PK obtained funding; designed, initiated and supervised the study. All authors were responsible for the intellectual content of the paper, and saw and approved the final version of the manuscript. 


\section{References}

1. van Haastregt JC, Diederiks JP, van Rossum E, de Witte LP, Crebolder HF. Effects of preventive home visits to elderly people living in the community: systematic review. BMJ 2000;320(7237):754-8.

2. Elkan R, Kendrick D, Dewey M, Hewitt M, Robinson J, Blair M, et al. Effectiveness of home based support for older people: systematic review and meta-analysis. BMJ 2001;323(7315):719-25.

3. Stuck AE, Egger M, Hammer A, Minder CE, Beck JC. Home visits to prevent nursing home admission and functional decline in elderly people: systematic review and meta-regression analysis. JAMA 2002;287(8):1022-8.

4. Meinck M, Lubke N, Lauterberg J, Robra BP. [Preventive home visits to the elderly: systematic review of available evidence]. Gesundheitswesen 2004;66(11):732-8.

5. Hendriksen C, Lund E, Stromgard E. Consequences of assessment and intervention among elderly people: a three year randomised controlled trial. BMJ Clin res ed 1984;289(6457):1522-4.

6. van Rossum E, Frederiks CM, Philipsen H, Portengen K, Wiskerke J, Knipschild P. Effects of preventive home visits to elderly people. BMJ 1993;307(6895):27-32.

7. Stuck AE, Minder CE, Peter Wuest I, Gillmann G, Egli C, Kesselring A, et al. A randomized trial of in-home visits for disability prevention in community-dwelling older people at low and high risk for nursing home admission. Archives of Internal Medicine 2000;160(7):97786.

8. Rizzo JA, Baker DI, McAvay G, Tinetti ME. The cost-effectiveness of a multifactorial targeted prevention program for falls among community elderly persons. Med Care 1996;34(9):954-69.

9. Stuck AE, Aronow HU, Steiner A, Alessi CA, Bula CJ, Gold MN, et al. A trial of annual inhome comprehensive geriatric assessments for elderly people living in the community.[comment]. N Engl J Med 1995;333(18):1184-9.

10. Kronborg C, Vass M, Lauridsen J, Avlund K. Cost effectiveness of preventive home visits to the elderly : Economic evaluation alongside randomized controlled study. Eur J Health Econ 2006.

11. Hall N. Randomized trial of a health promotion program for frail elders. Canadian Journal on Aging/La Revue Canadienne du vieillissement 1992;11(1):72-91.

12. Pathy MS, Bayer A, Harding K, Dibble A. Randomised trial of case finding and surveillance of elderly people at home. Lancet 1992;340(8824):890-3.

13. Leveille SG, Wagner EH, Davis C, Grothaus L, Wallace J, LoGerfo M, et al. Preventing disability and managing chronic illness in frail older adults: a randomized trial of a community-based partnership with primary care.[comment]. J Am Geriatr Soc 1998;46(10):1191-8.

14. Robichaud L, Hébert R, Roy PM, Roy C. A preventive program for community-dwelling elderly at risk of functional decline: a pilot study. Arch Gerontol Geriatr 2000;30:73-84.

15. Nikolaus T, Bach M. Preventing falls in community-dwelling frail older people using a home intervention team (HIT): results from the randomized Falls-HIT trial. J Am Geriatr Soc 2003;51(3):300-5.

16. Yamada Y, Ikegami N. Preventive home visits for community-dwelling frail elderly people based on Minimum Data Set-Home Care: Randomized controlled trial. Geriatr Gerontol Intern 2003;3(4):236-242.

17. Markle-Reid M, Weir R, Browne G, Roberts J, Gafni A, Henderson S. Health promotion for frail older home care clients. J Adv Nurs 2006;54(3):381-95.

18. Dalby DM, Sellors JW, Fraser FD, Fraser C, van Ineveld C, Howard M. Effect of preventive home visits by a nurse on the outcomes of frail elderly people in the community: a randomized controlled trial. Canadian Medical Association Journal / Journal de l'Association Medicale Canadienne 2000;162(4):497-500. 
19. van Haastregt JC, Diederiks JP, van Rossum E, de Witte LP, Voorhoeve PM, Crebolder HF. Effects of a program of multifactorial home visits on falls and mobility impairments in elderly people at risk: randomised controlled trial.[comment]. BMJ 2000;321(7267):994-8.

20. Hebert R, Robichaud L, Roy PM, Bravo G, Voyer L. Efficacy of a nurse-led multidimensional preventive program for older people at risk of functional decline. A randomized controlled trial. Age and Ageing 2001;30(2):147-53.

21. Vass M, Avlund K, Lauridsen J, Hendriksen C. Feasible Model for prevention of Functional Decline in Older People: Municipality-Randomized, Controlled trial. J Am Geriatr Soc 2005;53:563-568.

22. Fletcher AE, Price GM, Ng ES, Stirling SL, Bulpitt CJ, Breeze E, et al. Population-based multidimensional assessment of older people in UK general practice: a cluster-randomised factorial trial. Lancet 2004;364(9446):1667-77.

23. van Rossum E. Effects of preventive home visits to the elderly. $\mathrm{PhD}$ Thesis Maastricht University, 1993.

24. Nicolaides-Bouman A, van Rossum E, Kempen GI, Knipschild P. Effects of home visits by home nurses to elderly people with health problems: design of a randomised clinical trial in the Netherlands [ISRCTN92017183]. BMC Health Serv Res 2004;4(1):35.

25. Nicolaides-Bouman A, van Rossum E, Habets H, Kempen GI, Knipschild P. Home visiting program for older people with health problems: process evaluation. J Adv Nurs 2007;58(5):425-35.

26. Bouman A, van Rossum E, Ambergen T, Kempen G, Knipschild P. Effects of a home visiting program for older people with poor health status: a randomised clinical trial in the Netherlands. J Am Geriatr Soc 2008;56(3):397-404.

27. Idler EL, Benyamini Y. Self-rated health and mortality: a review of twenty-seven community studies. J Health Soc Beh 1997;38(1):21-37.

28. Oostenbrink JB, Bouwmans CAM, Koopmanschap MA, Rutten FFH. Manual for costanalysis in health care research; The Health Care Insurance Board, Updated version 2004, 2004.

29. Oostenbrink JB, Koopmanschap MA, Rutten FFH. Standardisation of costs: the Dutch Manual for Costing in Economic Evaluations. Pharmacoeconomics 2002;20(7):443-454.

30. Briggs A, Wonderling D, Mooney C. Pulling cost-effectiveness analysis up by its bootstraps: a non-parametric approach to confidence interval estimation. Health Econ 1997;6:327-340.

31. [http://www.who.int/classifications/atcddd/en/]. Last accessed 23 January 2008.

32. Bernabei R, Landi F, Gambassi G, Sgadari A, Zuccala G, Mor V, et al. Randomised trial of impact of model of integrated care and case management for older people living in the community. BMJ Clin res ed 1998;316(7141):1348-51.

33. van den Brink M, van den Hout WB, Stiggelbout AM, van de Velde CJ, Kievit J. Cost measurement in economic evaluations of health care: whom to ask? Med Care 2004;42(8):740-6 



\title{
6
}

\section{Effects of intensive home visiting programs for older people with poor health status: a systematic review}

\author{
Ans Bouman ${ }^{1}$, Erik van Rossum ${ }^{24}$, Patricia Nelemans ${ }^{1}$, \\ Gertrudis Kempen $^{2}$ and Paul Knipschild ${ }^{3}$
}

BMC Health Serv Res 2008, 8:74

1 Department of Epidemiology, Maastricht University, Maastricht, the Netherlands

2 School for Public Health and Primary Care, Maastricht University, Maastricht, the Netherlands

3 Department of General Practice, Maastricht University, Maastricht, the Netherlands

4 Centre on Autonomy and Participation of the chronically ill, Zuyd University, Heerlen, the Netherlands 


\begin{abstract}
Background

Home visiting programs have been developed aimed at improving the health and independent functioning of older people. Also, these intend to reduce hospital and nursing home admission and associated cost. A substantial number of studies have examined the effects of preventive home visiting programs on older people living in the community; the findings have been inconsistent. The objective of this review was to assess the effectiveness of intensive home visiting programs targeting older people with poor health or otherwise with functional impairments.
\end{abstract}

\title{
Methods
}

A search for literature was based on included trials from four reviews on the effectiveness of home visits published after 2000 and on a database search of Cinahl, the Cochrane Central Register of Controlled Trials, Embase, Medline and PsycINFO from 2001 onwards. We also manually searched reference lists from potentially relevant papers. Randomized controlled trials were included assessing the effectiveness of intervention programs consisting of at least four home visits per year, an intervention duration of 12 months or more, and targeting older people (aged 65 years and over) with poor health. Two reviewers independently abstracted data from full papers on program characteristics and outcome measures; they also evaluated the methodological quality.

\section{Results}

The search identified 844 abstracts; eight papers met the inclusion criteria. Seven trials were of sufficient methodological quality; none of the trials showed a significant favorable effect for the main analysis comparing the intervention group with the control group on mortality, health status, service use or cost. The inclusion of less-intensive intervention programs for frail older persons would not have exerted a great influence on the findings of our review.

\section{Conclusions}

We conclude that home visiting programs appear not to be beneficial for older people with poor health within the health care setting of Western countries. 


\section{Background}

Home visiting programs have been developed aimed at improving the health and independent functioning of older people. Also, these intend to reduce hospital and nursing home admission and associated cost. A substantial number of studies have examined the effects of preventive home visiting programs on older people living in the community. Since 2000 four systematic reviews ${ }^{1-4}$, a literature review ${ }^{5}$ and a synthesis of several reviews ${ }^{6}$ have been published. Furthermore, a review on the effectiveness of preventive primary care outreach interventions aimed at older people was published, but this review also included trials not based on home visiting programs ${ }^{7}$. The reviews produced inconsistent and conflicting results. Subgroup analyses of the largest meta-analysis showed that effective home-visiting programs include multidimensional assessment, many follow-up visits and targeted people at lower risk of death. ${ }^{3}$

A trial in the Netherlands in the 1990s showed that home visits do not seem to be useful for the general population of older people, but subgroup analyses in this study suggested benefits for older people with poor health. ${ }^{89}$ To investigate this further, a Dutch trial by Bouman et al. was recently conducted focusing on older people with poor health at baseline. ${ }^{10-13}$ The study could not confirm beneficial effects on health status or service use for this target group. It is possible however that these findings constituted an isolated observation. In order to make a more well-founded judgment upon the effectiveness of home visits for this group of older persons, we decided to integrate the evidence from the study by Bouman et al. with evidence from other trials. Here, we publish a systematic review to investigate whether the findings from this trial have also been reported by comparable studies targeting older people with poor health, or otherwise with functional impairments ('frail older persons'). Insight into whether home visiting programs for older people with poor health are effective is essential for implementation and future research. An assessment of the methodological quality of the included trials is also presented.

\section{Methods}

\section{Search strategy}

The search for trials until 2001 was based on the included trials from four systematic reviews (Table 1). ${ }^{1-4}$ These reviews consider a total of 30 trials. The systematic review by Ploeg et al. ${ }^{7}$ was not included; none of the additional trials in this review were based on home visiting programs. Because the largest review and meta-analysis by Stuck et al. was based on an electronic search until 2001, we decided to continue our database search from this year onwards. ${ }^{3}$ On July 11 2007, we searched the following databases: Cinahl, the Cochrane Central Register of Controlled Trials, Embase, Medline and PsycINFO. The following terms had to be used in the abstract or title: 'geriatric assessment', 
'home visit*', 'health visit*', or 'health screening', in combination with 'prevent*' or 'screen*'. Because we were only interested in randomized controlled trials for an older population, we also included in the search the exploded medical subject heading (MeSH) terms 'randomized' and 'aged'; this restricted search with MeSH terms was, however, not possible within all databases. Reference lists from potentially relevant papers were manually searched for additional studies. A search for unpublished data was carried out for studies that had published a (design) article of their home visiting program, but without available outcome data or lacking other information necessary to determine eligibility. No language restrictions were imposed.

\section{Selection}

We included randomized controlled trials examining the effects of home visiting programs for people aged 65 years and over. Based on earlier descriptions, preventive home visits are defined as visits to older people living in the community, which are aimed at multidimensional medical, functional, psychosocial, and environmental evaluation of their problems and resources. ${ }^{135}$ This evaluation results in specific recommendations aimed at reducing or treating the observed problems and preventing new ones. Follow-up visits are included for the implementation of the intervention plan.

The target populations were older people with a poor health status based on either subjective (e.g. self-rated health) or more 'objective' measures (e.g. (selfreported) functional impairments, and dependencies in (instrumental) activities of daily living). It has been suggested that higher risk older persons would benefit most from a more-intensive intervention that includes systematic followup and coordination as well as more frequent visits. ${ }^{14-16}$ If we were to expect benefits for this target population, a more intensive intervention may be a necessary requirement. We therefore decided to include studies with a relatively long and intensive follow-up, that is, when the intervention programs consisted of at least four home visits per year and the duration of the follow-up home visit period lasted 12 months or more; the home visits were to be carried out by health professionals, e.g. nurses or general practitioners. Since we focused on older people with poor health, who mostly suffer from multiple health problems, we excluded studies targeted at people with one specific disease. Studies without available data on health status, service use or cost were also excluded. 
Table 1 Included trials in systematic reviews on home visiting programs

\begin{tabular}{|c|c|c|c|c|c|}
\hline & & $\begin{array}{c}\text { Haastregt }^{1} \\
2000\end{array}$ & $\begin{array}{c}\text { Elkan }^{2 *} \\
2001\end{array}$ & $\begin{array}{l}\text { Stuck }^{3} \\
2002\end{array}$ & $\begin{array}{c}\text { Meinck }^{4 \dagger} \\
2004\end{array}$ \\
\hline & & \multicolumn{4}{|c|}{ Electronic search } \\
\hline Author(s) & Year & 1966-1999 & $\begin{array}{l}1966- \\
1997\end{array}$ & $\begin{array}{l}1985- \\
2001\end{array}$ & up to 2003 \\
\hline Luker $^{48}$ & 1981 & $x$ & $x$ & & \\
\hline Gunner et al. ${ }^{49}$ & 1984 & & & $x$ & $x$ \\
\hline Vetter et al. ${ }^{50 \ddagger}$ & 1984 & $x$ & $x$ & $x$ & $x$ \\
\hline Hendriksen et al. ${ }^{51}$ & 1984 & $x$ & $x$ & $x$ & $x$ \\
\hline Sorensen et al. ${ }^{52}$ & 1988 & $x$ & & $x$ & $x$ \\
\hline Balaban et al. ${ }^{33}$ & 1988 & & $x$ & & $x$ \\
\hline Carpenter et al. ${ }^{53}$ & 1990 & $x$ & & $x$ & $x$ \\
\hline McEwan et al. ${ }^{54}$ & 1990 & $x$ & $x$ & $x$ & $x$ \\
\hline Oktay et al. ${ }^{55}$ & 1990 & & $x$ & & $x$ \\
\hline Clarke et al. ${ }^{56}$ & 1992 & & & $x$ & $x$ \\
\hline Hall $^{20}$ & 1992 & $x$ & $x$ & & $x$ \\
\hline Pathy et al. ${ }^{32}$ & 1992 & $x$ & $x$ & $x$ & $\mathrm{x}$ \\
\hline Hansen et al. ${ }^{30}$ & 1992 & & $x$ & & $x$ \\
\hline Williams et al. ${ }^{27}$ & 1992 & & $x$ & & $x$ \\
\hline Vetter et al. ${ }^{57}$ & 1992 & $x$ & & $x$ & $x$ \\
\hline van Rossum et al. ${ }^{89}$ & 1993 & $x$ & $x$ & $x$ & $x$ \\
\hline Dunn et al. ${ }^{34}$ & 1994 & & $x$ & & $x$ \\
\hline Fabacher et al. ${ }^{58}$ & 1994 & $x$ & $x$ & $x$ & $x$ \\
\hline Tinetti et al. ${ }^{29}$ & 1994 & $x$ & & $x$ & $x$ \\
\hline Wagner et al. ${ }^{59}$ & 1994 & $x$ & & & $x$ \\
\hline Archbold et al..$^{60}$ & 1995 & & $x$ & & $x$ \\
\hline Stuck et al. ${ }^{61}$ & 1995 & $x$ & $x$ & $x$ & $x$ \\
\hline Dalby et al. ${ }^{21}$ & 2000 & & & & $x$ \\
\hline Stuck et al. ${ }^{15}$ & 2000 & & & $x$ & $x$ \\
\hline van Haastregt et al..$^{23} 24$ & 2000 & & & $x$ & $x$ \\
\hline Hebert et al. ${ }^{62}$ & 2001 & & & $x$ & $x$ \\
\hline Newbury et al. ${ }^{63}$ & 2001 & & & $x$ & $x$ \\
\hline Gill et al. ${ }^{44}$ & 2003 & & & & $x$ \\
\hline Yamada et al. $^{22}$ & 2003 & & & & $x$ \\
\hline
\end{tabular}

* Evaluates home visiting programs that offer health promotion and preventive care; includes nonrandomized controlled trials.

$\dagger$ Based on the previous reviews with addition of three trials.

$\ddagger$ One study describing two trials. 


\section{Validity assessment}

The quality of the research methods was evaluated using an adaptation of the Cochrane Back Review Group list of criteria. ${ }^{17}$ Four items were omitted, because these were used as inclusion criteria (random allocation and relevance of outcome measure) or were not applicable to the evaluated interventions (blinding of the participants and care provider). The criteria list consists of five descriptive, two statistical, and eight validity items. Each item was scored ' + ' if the criterion was fulfilled, '-' if the criterion was not fulfilled, and '?' if the information was not provided or was unclear. Scores on validity items ranged from 0 to 8 per trial. Trials with at least four fulfilled validity items were considered to be of 'sufficient methodological quality'; ${ }^{18}$ only those were included in the evaluation of the effectiveness. The items were scored independently by two reviewers ( $\mathrm{AB}$ and $\mathrm{PN})$. Disagreement was resolved by consensus or a third party (EvR).

\section{Data abstraction}

Titles and abstracts resulting from the database search were independently screened ( $\mathrm{AB}$ and $\mathrm{PK})$. Full papers were obtained for potentially relevant studies. Data from all relevant papers were independently abstracted (AB and $\mathrm{PN}$ ); this included data on characteristics of the home visiting programs and outcomes of the trials. Qualitative data abstraction was performed because of heterogeneity between trials regarding interventions and outcome measures. If information was absent from the original paper, attempts were undertaken to obtain complete information from the authors. Reviewers were not blinded to authors' names or institutions or journal of publication. Disagreement was resolved by consensus or a third party (EvR).

\section{Results}

\section{Trial flow}

Eight hundred and forty-four abstracts were identified, of which 234 duplicates were discarded (Figure 1). After screening whether the papers referred to randomized controlled trials that investigated home visiting programs for populations aged 65 years and over, we excluded another 542 abstracts. The remaining potentially relevant papers $(n=68)$ were further screened for population and intervention characteristics. Most papers were excluded because the intervention did not consist of at least four visits per year or the duration of the intervention period was less than 12 months $(n=46)$. Another 14 papers were excluded because the target population did not consist of older people with poor health $(n=11)$, the interveners were volunteers $(n=2)$, or there were no outcomes available $(n=1)$. Eight trials met the inclusion criteria; six of these have already 
been described in the previous systematic reviews and two studies were newly added. Design articles had been published of the two new studies; for the trial by van Hout et al. ${ }^{19}$ no published outcomes were available and the outcomes from the trial by Bouman et al. ${ }^{10}$ had been accepted for publication. ${ }^{12}{ }^{13} \mathrm{We}$ contacted van Hout et al. for available data on the results of their study; we received information to complete Tables 2, 3 and 4 (see below), but they could not supply estimates of their data to complete Table 5 (see below), because those were due for publication elsewhere.

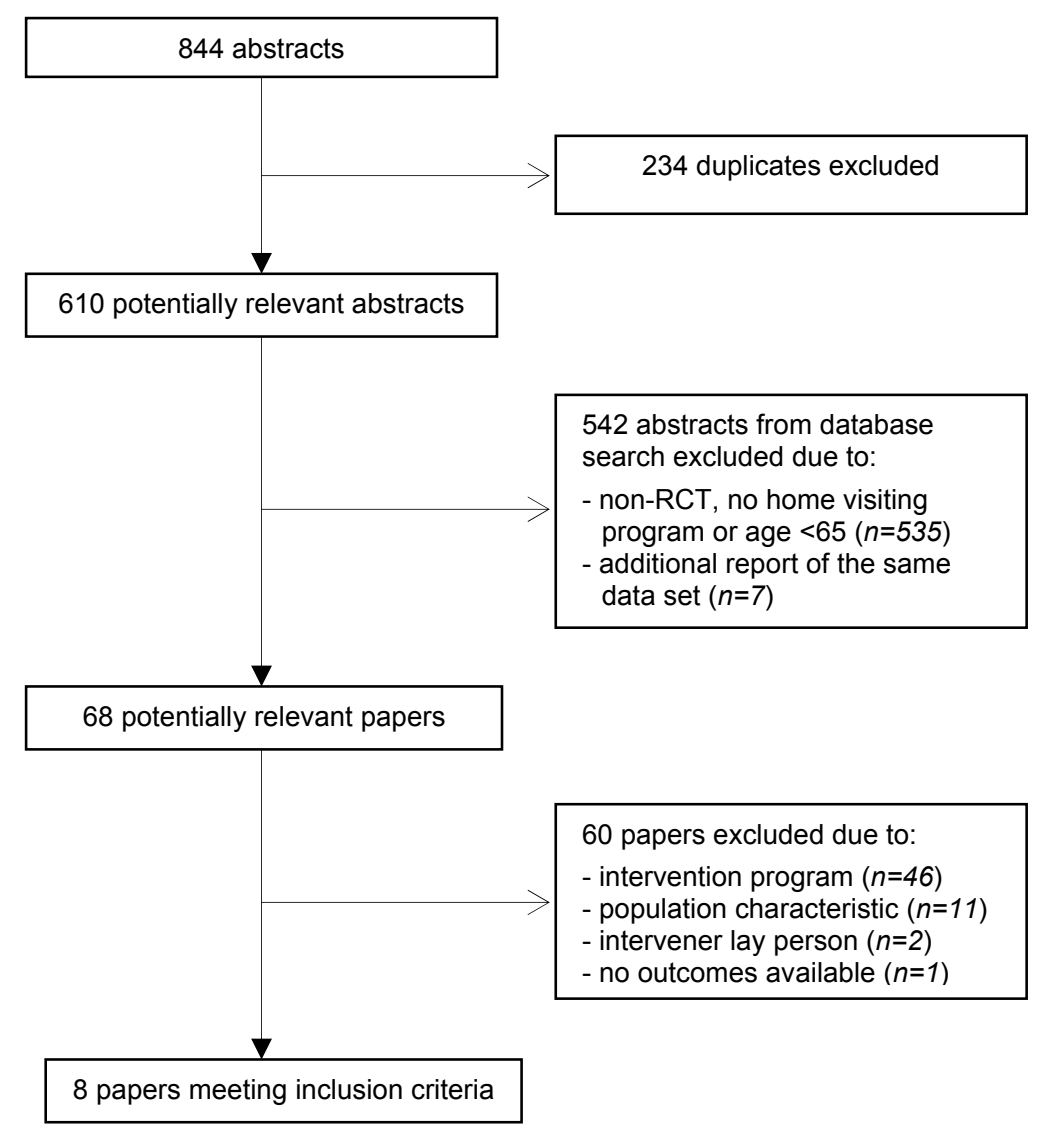

Figure 1 Progress of search for relevant trials

\section{Validity assessment}

Table 2 presents the results of the methodological quality assessment of the eight trials meeting the inclusion criteria. The observed sum score on the validity items ranged from 3 to 7 points (out of range $0-8$ points). Only the trial 
by $\mathrm{Hall}^{20}$ did not fulfill at least four criteria and was considered to be of insufficient methodological quality; the other seven trials were of sufficient methodological quality. Data on whether co-interventions were avoided or comparable between the intervention and control group, or whether the compliance was acceptable, was often not provided. For three trials the study groups were not similar at baseline. ${ }^{1520} 21$ Most papers, however, reported a concealment of treatment allocation, blinding of the outcome assessor to the intervention and inclusion of an intention-to-treat analysis.

\section{Study characteristics}

Characteristics of six home visiting programs are shown in Table 3. Two trials were not tabulated, because one was of insufficient methodological quality ${ }^{20}$ and another presented only a subgroup with poor health from the studied general population of older people. ${ }^{8}$ The targeted populations were people aged 65 years and over with poor self-reported health (in the trials by van Hout et $a l .{ }^{19}$ and Bouman et al. ${ }^{12}{ }^{13}$ ); with instrumental activities of daily living (IADL) dependencies (in the trial by Yamada et $a{ }^{22}{ }^{22}$ ); or otherwise with functional impairments (in the trials by Dalby et al. ${ }^{21}$, Stuck et al. ${ }^{15}$ and van Haastregt et $a .^{23}{ }^{24}$ ). The trial by Yamada et al. also included a subgroup of older people with poor self-reported health from the original population with IADL dependencies; ${ }^{22}$ this subgroup is not tabulated. The mean age of the study populations was mostly between 75-79 years. In one trial, the sample size was relatively small with 73 intervention and 69 control participants. ${ }^{21}$ Most trials offered four or five home visits per year, with an intervention duration varying between 12 and 36 months. The mean number of visits was $4.5^{25}, 5.1^{22}, 7.3^{11}$, $7.5^{15}$, and, on average, 18.9 hours (from personal communication with the first author). ${ }^{21}$ The visits were carried out by home or health nurses; in two trials the assessment was done in cooperation with a primary care physician ${ }^{19}{ }^{21}$ and in another trial with a geriatrician. ${ }^{15}$ Compliance with the recommendations was reported in half of the trials, varying between $46 \%$ and $65 \%$.

\section{Outcome measures}

None of the trials with sufficient methodological quality showed a significant favorable effect for the main analysis comparing the intervention group with the control group on mortality, health status, service use or cost (Table 4). ${ }^{12} 13151921$ -

${ }^{24}$ For two trials, follow-up of outcome measurements were available at the end of the intervention period and 6 months thereafter. ${ }^{12}{ }^{23}$ Van Haastregt et al. found a favorable effect for the intervention group compared to the control group on functional status at the end of the intervention period (12 months), but this effect had disappeared after 18 months of follow-up. ${ }^{23}$ None of the other outcomes in this study showed a beneficial effect of the program at 12 or 18 months of follow-up. In the study by Bouman et al. none of the outcomes showed statistically significant differences between the intervention and control 
group for follow-up measurements at 18 months (the end of the intervention period) or at 24 months. ${ }^{12}$ The study by Stuck et al. included a home visit follow-up period of 24 months, but the outcome follow-up was measured at 36 months; no differences in favor of the intervention group compared with the control group were demonstrated. ${ }^{15}$

The post hoc subgroup analyses (analyses done after looking at the data) of the trial by van Rossum ${ }^{89}$ yielded positive effects for intervention participants on several outcome measures, e.g. on health status, IADL, admissions to hospital and homes for older persons, and mortality; the post hoc subgroup analyses of Yamada et $a l .{ }^{22}$ showed favorable effects for activities of daily living (ADL) and mental health. The one trial of insufficient methodological quality reported a favorable effect for the intervention group compared to the control group on nursing home admissions (not tabulated). ${ }^{20}$

For the most commonly measured outcomes shown in Table 4 (functional status, hospital and nursing home admissions, and mortality), more detailed information is provided in Table 5. The data on health status is not shown in this table, because the outcomes were based on a variety of different measurement instruments and these could not be reduced to a common denominator. For patients in the intervention and control group, respectively, means or percentages are presented - with unfavorable outcomes on mortality, functional status, and hospital and nursing home admissions. No further quantitative data synthesis or pooling was carried out because of the limited number of studies. 


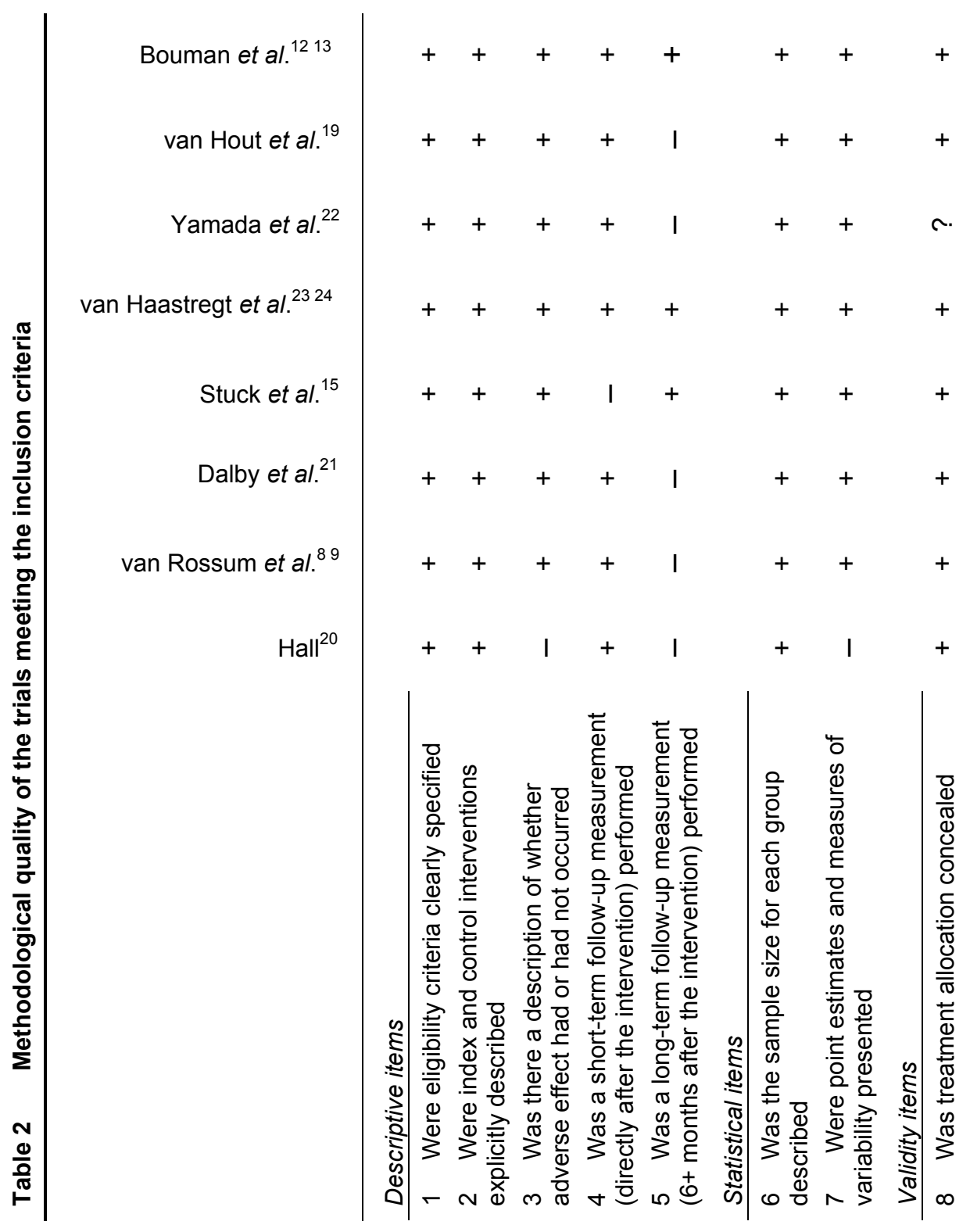




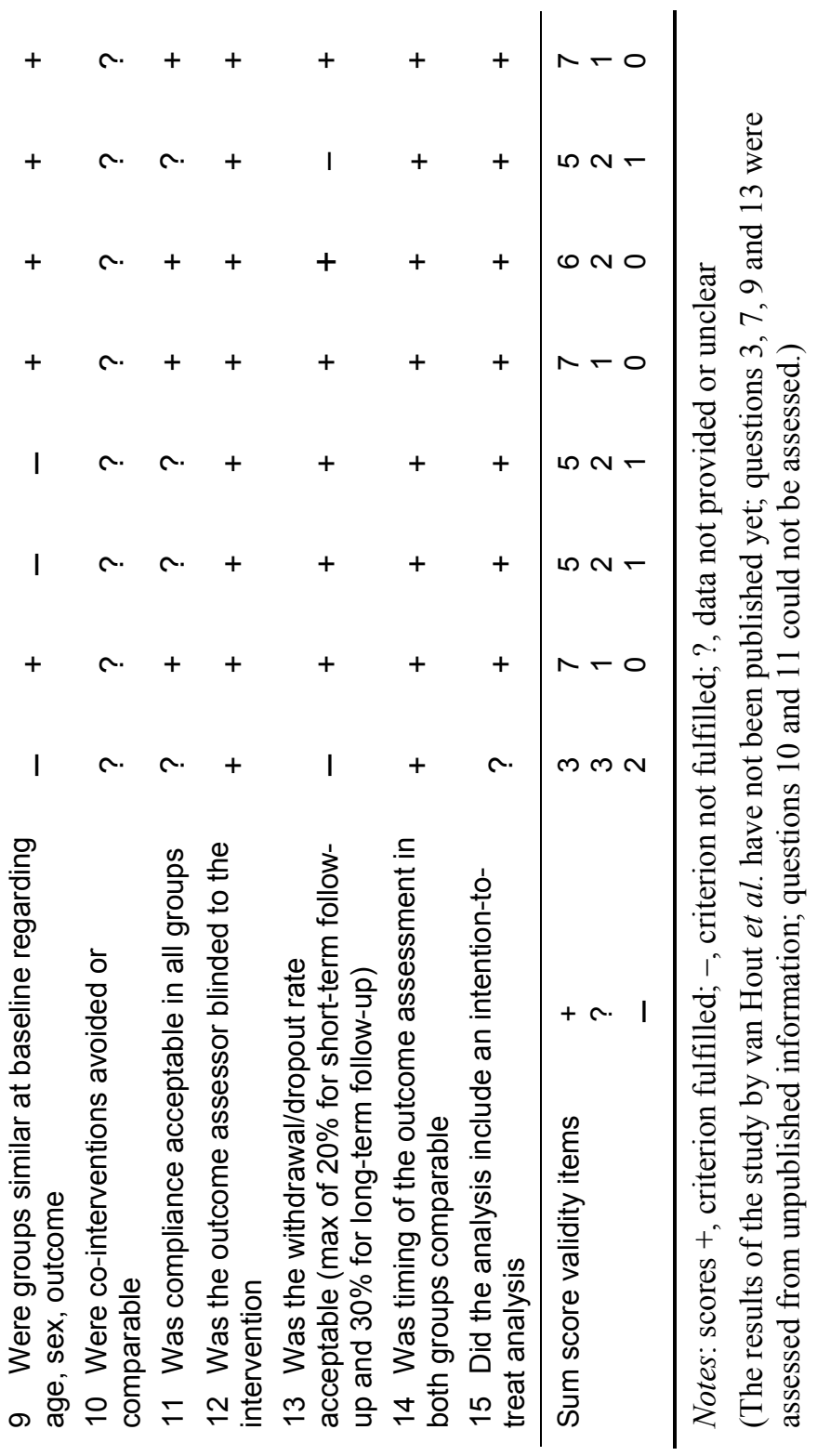


Chapter 6

Table 3 Characteristics of the included home visiting programs

\begin{tabular}{|c|c|c|c|}
\hline $\begin{array}{l}\text { Author(s) } \\
\text { Year } \\
\text { Country }\end{array}$ & $\begin{array}{l}\text { Sample } \\
\text { size } \\
\mathrm{nr} \text { I/C }\end{array}$ & Health status participants & $\begin{array}{l}\text { Mean } \\
\text { age }\end{array}$ \\
\hline $\begin{array}{l}\text { Dalby et al. } \\
2000 \\
\text { Canada }\end{array}$ & $73 / 69$ & $\begin{array}{l}\text { self-reported functional impairment, or } \\
\text { admission to hospital or bereavement in the } \\
\text { previous } 6 \text { months }\end{array}$ & 79 \\
\hline $\begin{array}{l}\text { Stuck et al. } \\
2000 \\
\text { Switzerland }\end{array}$ & $116 / 231$ & $\begin{array}{l}\text { high-risk status based on six baseline } \\
\text { predictors of functional deterioration }\end{array}$ & $82^{\dagger}$ \\
\hline $\begin{array}{l}\text { van Haastregt et al. }{ }^{2324} \\
2000 \\
\text { Netherlands }\end{array}$ & $159 / 157$ & $\begin{array}{l}\text { moderate impairments in mobility, score } \geq 3 \text { on } \\
\text { mobility control scale of the short-version } \\
\text { sickness impact profile, or a history of recent } \\
\text { falls ( } \geq 2 \text { in previous } 6 \text { months) }\end{array}$ & 77 \\
\hline $\begin{array}{l}\text { Yamada et al. } \\
222 \\
\text { Japan }\end{array}$ & $184 / 184$ & $\begin{array}{l}\text { dependent in IADL, independent in ADL, and } \\
\text { not rating their health as excellent }\end{array}$ & 79 \\
\hline $\begin{array}{l}\text { van Hout et al. } \\
2005 \\
\text { Netherlands }\end{array}$ & $331 / 320$ & $\begin{array}{l}\text { self-reported health score in the worst quartile } \\
\text { of at least two of six COOP-WONCA charts }\end{array}$ & $\geq 75$ \\
\hline $\begin{array}{l}\text { Bouman et al. }{ }^{1011} \\
2007 \\
\text { Netherlands }\end{array}$ & $160 / 170$ & $\begin{array}{l}\text { self-reported poor health status at baseline, } \\
\text { score } 1-5 \text { on a scale from } 1-10 \\
\text { (very poor-excellent health) }\end{array}$ & 76 \\
\hline
\end{tabular}

Notes: I, intervention group; C, control group; ADL, activities of daily living; LTC, Long Term Care; MDS-HC, minimal data set home care; COOP-WONCA, COOP functional health assessment charts; RAI-HC, resident assessment inventory home care

* The control group received usual care.

$\dagger$ Mean over entire group of high-risk and low-risk older persons. 
Table 3 continued

\begin{tabular}{|c|c|c|c|c|}
\hline Intervention program* & $\begin{array}{l}\text { Number of } \\
\text { visits per } \\
\text { year }\end{array}$ & $\begin{array}{l}\text { Duration of } \\
\text { intervention } \\
\text { in years }\end{array}$ & Intervener & Compliance \\
\hline $\begin{array}{l}\text { multidimensional assessment; } \\
\text { a care plan was developed } \\
\text { together with the primary care } \\
\text { physician }\end{array}$ & $\begin{array}{l}\text { as needed } \\
\text { (mean } 18.9 \\
\text { hours) }\end{array}$ & 1.2 & $\begin{array}{l}\text { primary care } \\
\text { nurse }\end{array}$ & not reported \\
\hline $\begin{array}{l}\text { annual multidimensional } \\
\text { assessment (with physical } \\
\text { examinations); preventive } \\
\text { home visits in collaboration with } \\
\text { the project team's geriatricians }\end{array}$ & $\begin{array}{c}4 \\
\left(\text { mean } 7.5^{\dagger}\right)\end{array}$ & 2 & $\begin{array}{l}\text { trained public } \\
\text { health nurse }\end{array}$ & not reported \\
\hline $\begin{array}{l}\text { multidimensional assessment } \\
\text { with checklists and use of } \\
\text { guidelines; systematic home } \\
\text { visits }\end{array}$ & $\begin{array}{c}5 \\
\text { (mean 4.5) }\end{array}$ & 1 & $\begin{array}{l}\text { trained } \\
\text { community } \\
\text { health nurse }\end{array}$ & $\begin{array}{l}46 \% \text { for referrals } \\
\text { and advice }\end{array}$ \\
\hline $\begin{array}{l}\text { multidimensional assessment } \\
\text { based on the MDS-HC; } \\
\text { scheduled home visits, primary } \\
\text { objective human interaction }\end{array}$ & $\begin{array}{c}4 \\
\text { (mean 5.1) }\end{array}$ & 1.5 & $\begin{array}{l}\text { trained public } \\
\text { health nurse }\end{array}$ & $47 \%$ for advice \\
\hline $\begin{array}{l}\text { multidimensional assessment } \\
\text { with RAI-HC; systematic home } \\
\text { visits, an individual care plan } \\
\text { was set up complying with } \\
\text { patient priorities together with } \\
\text { the primary care practice }\end{array}$ & 5 & 1.5 & $\begin{array}{l}\text { trained home } \\
\text { nurse }\end{array}$ & not (yet) reported \\
\hline $\begin{array}{l}\text { multidimensional assessment } \\
\text { with EasyCare questionnaire } \\
\text { and checklists; systematic } \\
\text { home visits, individual plan in } \\
\text { agreement with the older } \\
\text { persons }\end{array}$ & $\begin{array}{c}5.3 \\
(\text { mean } 7.3)\end{array}$ & 1.5 & $\begin{array}{l}\text { trained home } \\
\text { nurse }\end{array}$ & $\begin{array}{l}65 \% \text { for referrals } \\
58 \% \text { for advice }\end{array}$ \\
\hline
\end{tabular}




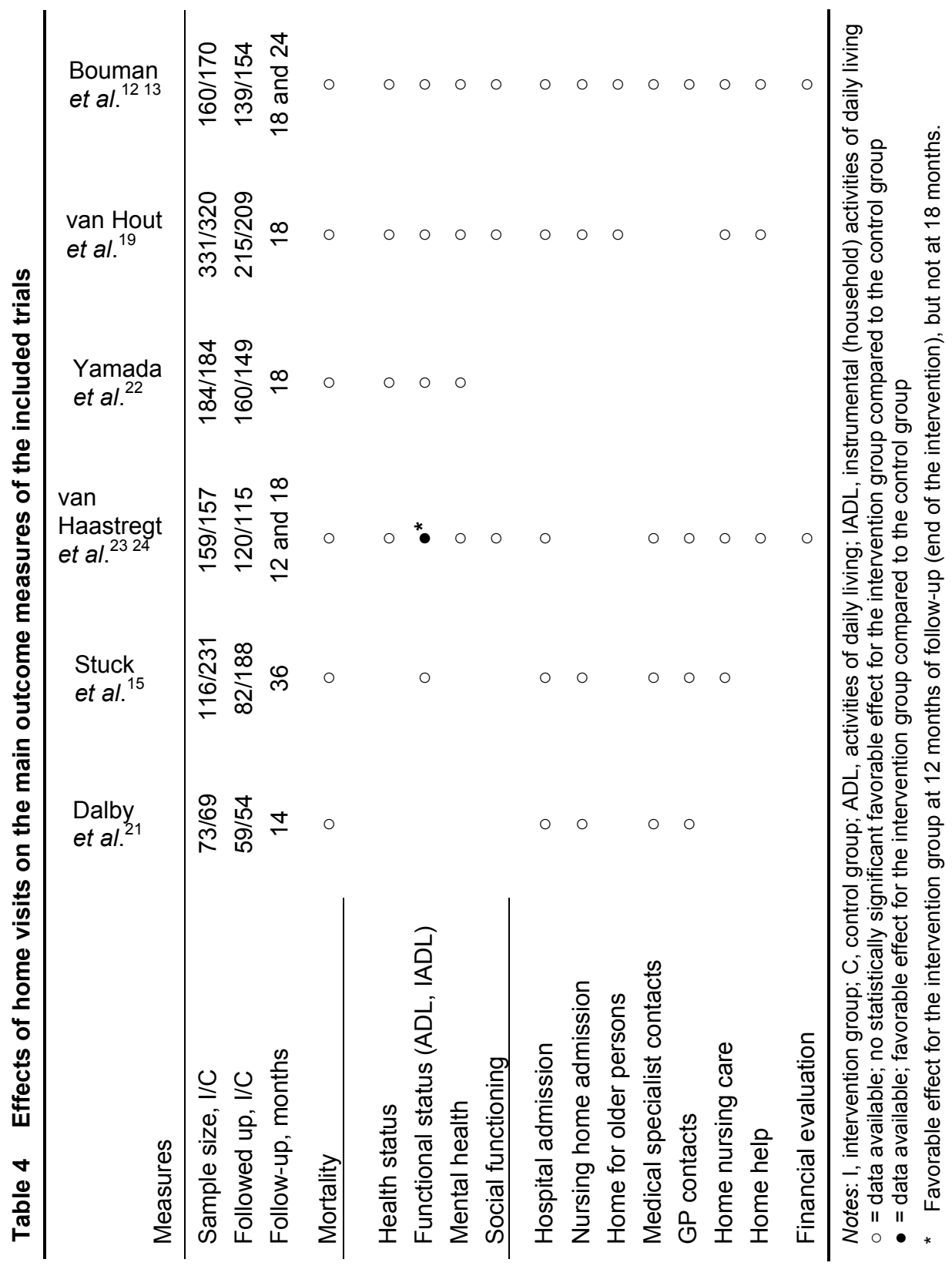




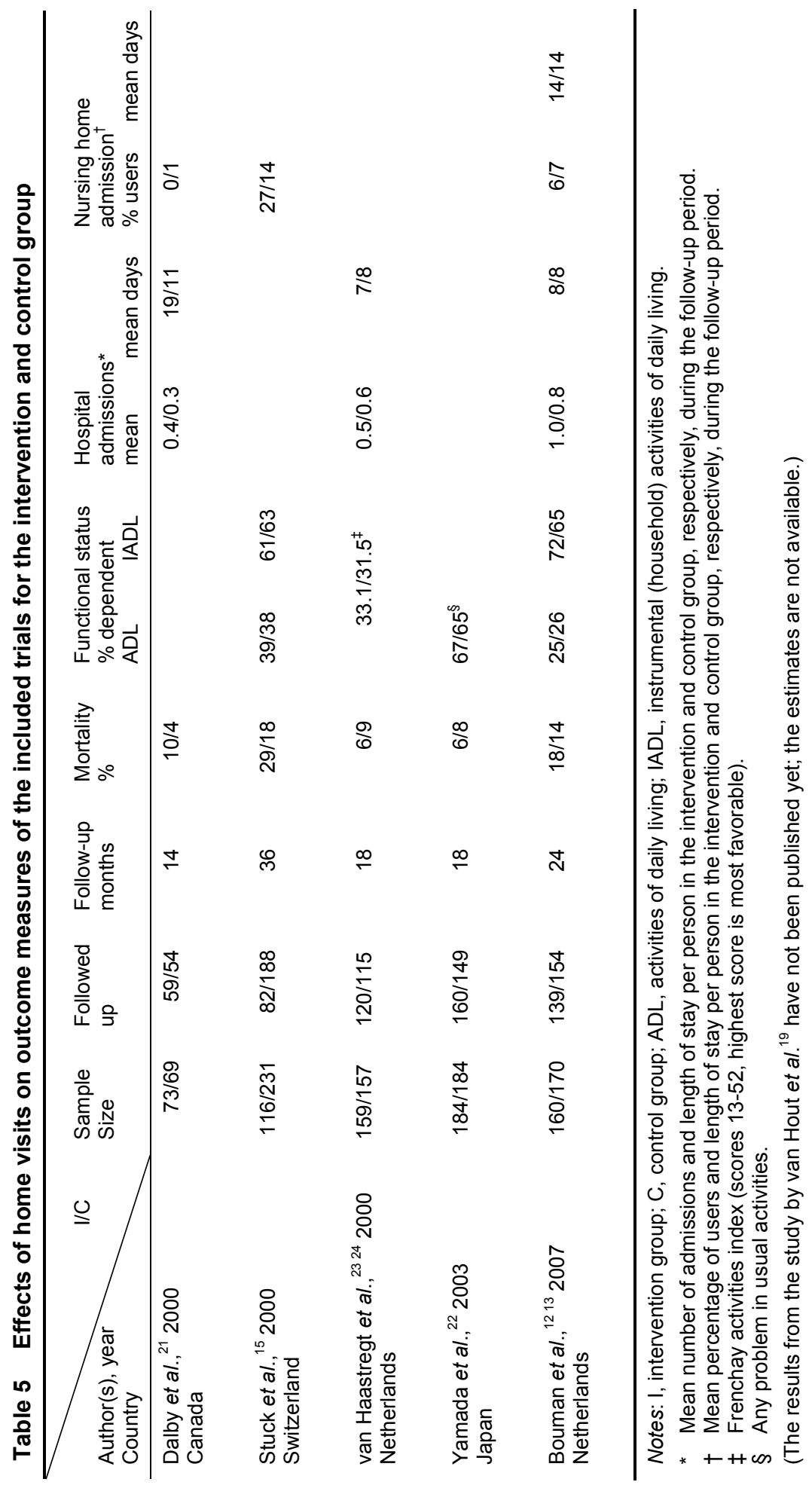




\section{Discussion}

Of the eight randomized controlled trials evaluating the effectiveness of intensive home visiting programs for older people with poor health or otherwise with functional impairments, seven were of sufficient methodological quality according to the predefined standard. Even though nearly all studies were methodologically sound, improvements are still possible in specifying whether co-interventions were avoided or comparable between the intervention and control group, and, for half of the studies, in reporting the compliance with the interventions. The main analysis results from the trials of sufficient methodological quality consistently showed that the home visiting programs had no effect on the health status or service use of older people with poor health. Based on the information provided in these studies, also no differences were found between the intervention and control group in mortality.

In our evaluation, we did not include the results from the trial of insufficient methodological quality ${ }^{20}$ and from post hoc subgroup analyses within two trials. $^{822}$ These studies reported some positive effects, but the results were based on small sample sizes, and, in the trial of insufficient methodological quality, the outcomes may also have been affected by the large drop-out rate and the dissimilarity of the study groups at baseline. The positive effects from the post hoc subgroup comparison from the earlier Dutch study ${ }^{8}$ that indicated the visits to be effective for those with a poor perceived health status at baseline could not be confirmed by the results from a larger replication study. ${ }^{12}$

The review consists largely of published information; some unpublished data was obtained from two systematically searched studies. ${ }^{19}{ }^{21}$ We did not further supplement the database search with expert consultations and it is possible that unpublished data from other trials is available. Studies with significant, positive, results are generally easier to find than those with non-significant or negative results and this could lead to a bias toward a more positive result. ${ }^{26}$ Since nearly all studies in our review have negative results, publication bias can not explain the results. To ensure an acceptable standard for design and quality, only randomized controlled trials were included and an assessment of validity items was provided. It is rather striking that the one trial, that provided positive results, was considered to be of poor methodological quality by both reviewers.

In the review by van Haastregt et al. ${ }^{1}$ no clear evidence was found in favor of the effectiveness of preventive home visits to older persons. The review and meta-regression analysis by Stuck et al. ${ }^{3}$, including 11/15 of the studies from van Haastregt et al. (see Table 1), also showed no effect on mortality, nursing home admissions or functional status. Both reviews included trials with different target populations and interventions. Meta-analytic subgroup analyses in the review by Stuck et al. suggested, however, benefits from home visiting programs for certain types of patients or interventions. For instance, when interventions were targeted at persons with a lower risk for death (defined as 
annual mortality rates between $3 \%$ and $6 \%$ ), a reduction in functional decline was shown. No reductions were shown for target populations with a mortality rate above $6 \%$. This latter finding is in agreement with the findings in this review, wherein mortality rates were above $6 \%$ in most studies. Meta-analyses in the review by Elkan et al. ${ }^{2}$, including 8/15 studies from van Haastregt et al., also focused on subgroups of frail older persons: no effects were shown on functional status, but positive effects were shown on mortality and nursing home admissions. However, these findings for frail older persons were based on only four studies, including one nonrandomized controlled trial ${ }^{27}$ and one we scored in our review as being of insufficient methodological quality. ${ }^{20}$

From the search, a number of home visiting programs targeting frail older persons were not included in our review because the intervention consisted of less than four visits per year and/or the duration of the intervention program was shorter than 12 months. The results of studies on less-intensive interventions are mixed. Five studies out of nine showed positive results: two studies with benefits for the intervention group on fall-related outcomes; ${ }^{28}{ }^{29}$ one study showing more nursing home admissions in the control group; ${ }^{30}$ one study showing improvements in psychosocial functioning for the intervention group; ${ }^{31}$ and one study demonstrating a shorter length of stay at the hospital in the intervention group in the younger group (aged 65-74), but not in the older group (aged 75 or older), and better self-rated health scores in the intervention group (no baseline measurements were available). ${ }^{32}$ Four studies out of nine had negative results. ${ }^{33-36}$ As most positive trials (5/9) showed benefits for only one parameter, while the other trials were negative (4/9), the overall benefits seem limited. In case we had used different inclusion criteria and added these studies, this would not have exerted a great influence on the findings of our review.

The mean number of visits of the trials presented in Table 3 ranged from 4.5 to 7.5, and, on average, 18.9 hours; for the non-included studies targeting frail older persons this ranged from 1 to approximately $3,{ }^{28} 30$ 32-36 with the exception of 7.8 visits in the Tinetti et al. study ${ }^{29}$ and a median of 5 visits in the MarkleReid et al. study. ${ }^{31}$ The last two studies were not included, because their intervention period lasted 6 months or less. In general, we succeeded in including studies with more-intensive programs. These did not, however, result in more favorable outcomes for the intervention group compared to the control group. Based on our definition of home visiting programs, all included trials reported a multidimensional assessment with follow-up, also with physical examination in the study by Stuck et al. ${ }^{15}$ Of the less-intensive non-included programs for frail older persons, Hebert et al. ${ }^{36}$ and Tinetti et al. ${ }^{29}$ included a multidimensional assessment with physical examination. There seemed no apparent relationship between characteristics of the assessments and beneficial effects of the programs. 
There is still debate on the frailty concept. The definition and identification of frail elderly varies considerably. ${ }^{37} 38$ As there is no consensus yet, we used 'poor health status' as inclusion criteria, but we also referred to 'frail older persons'. Because of the wide use in defining frail populations, we decided that the inclusion could be based on either subjective measures (e.g. self-rated health, which is an overall measure for functional health abilities, including physical, mental and social functioning and has shown predictive validity for mortality and institutionalization among older persons ${ }^{9}{ }^{39}$ ) or on more 'objective' measures (e.g. ADL-scores). Also other measures, e.g. 'at risk for falls' or 'functional impairments', have been described for target populations of frail older persons, and these studies were eligible. The non-included home visiting studies also targeted frail older persons, but did not match our criteria on the intervention program.

The results of the trial by Bouman et al. in the Netherlands have been supported by all methodologically sound studies evaluated in this review. ${ }^{12}$ The results from the post hoc subgroup analysis from an earlier Dutch study on which this trial was based, could not be confirmed. ${ }^{8}$ The CONSORT statement ${ }^{40}$ already made notice of the risk of spurious findings from subgroup analyses, ${ }^{41}{ }^{42}$ and indicated that especially post hoc subgroup comparisons are likely not to be confirmed by further studies. This is in line with the findings of this review. Results from reported post hoc subgroup analyses should therefore be interpreted with great caution.

Contrary to our expectation, the findings of this review suggest that intensive home visiting programs targeted at older people with poor health status are not effective. As half of the included studies were carried out in the Netherlands and the other half in other Western countries, the results may only be applicable in comparable health care settings. There seem to be no arguments to add the home visiting program targeting older persons with poor health status to regular health care. In a number of countries, e.g. Japan, Denmark and Australia, preventive home visits are part of the national policy and the visits are incorporated into regular health care for older persons. It seems essential, that the programs are judged on their merit again when targeting older persons in poor health. The United Kingdom withdrew this policy in 2004 based on the results of a large national trial, which showed that different forms of multidimensional assessment, targeting either the general population of older persons or frail older persons, offered almost no differences in patient outcomes. ${ }^{35}$

Frail older persons may benefit from other types of interventions. In a recent editorial by Stuck and Kane, the authors indicated that older persons at higher risk or those already disabled are likely to benefit from multidimensional interventions that target specific problems, ${ }^{43}$ e.g. favorable effects were shown by a 6-month intensive home-based physical therapy program (16 visits over 6 months), ${ }^{44}$ a chronic disease self-management program including three visits and nine telephone calls, ${ }^{45}$ and by a nurse-centered discharge plan with follow- 
up home visits for frail older persons discharged from hospital. ${ }^{46}$ The economic arguments for these studies remain to be established however. Collaboration between different professionals involved may be necessary to manage the complex care that is often required by frail older persons. In a large European study (11 countries) it was found that home care service based on a case management approach reduced risk of institutionalization in a population of frail older persons in home care. ${ }^{47}$ Although care-coordination can be provided by a case-manager, ideally the health and social services should be more integrated and have coordination between the services to supply the best available options for the individual needs of older persons.

\section{Conclusions}

In conclusion, we think that intensive home visiting programs are probably not beneficial for frail older persons within the health care setting of Western countries. Since many older adults prefer to live in their own homes and the population of older adults, including frail adults, is expected to grow, future research is needed to search for alternative approaches to improve the health status of frail older persons.

\section{Authors' contributions}

EvR and PK developed the original idea. AB performed the search strategy, abstracted data, and drafted the manuscript; PN abstracted data. PK and EvR assisted in the data abstraction process. EvR, GK, PK and PN provided valuable comments during the process of writing this manuscript. All authors read and approved the final manuscript.

\section{Acknowledgements}

We thank Hein van Hout, VU University Medical Center Amsterdam, the Netherlands and Dawn Dalby, Wilfrid Laurier University, Waterloo, ON, Canada; they provided us with unpublished data.

The review is part of a study funded by ZonMw, the Netherlands Organization for Health Research and Development (Grant 22000115), The Hague, the Netherlands. The views expressed are the sole responsibility of the authors. The funding source had no influence on study design; in the collection and interpretation of the data; in the writing of the manuscript; and in the decision to submit the manuscript for publication. 


\section{References}

1. van Haastregt JC, Diederiks JP, van Rossum E, de Witte LP, Crebolder HF. Effects of preventive home visits to elderly people living in the community: systematic review. BMJ 2000;320(7237):754-8.

2. Elkan R, Kendrick D, Dewey M, Hewitt M, Robinson J, Blair M, et al. Effectiveness of home based support for older people: systematic review and meta-analysis. BMJ 2001;323(7315):719-25.

3. Stuck AE, Egger M, Hammer A, Minder CE, Beck JC. Home visits to prevent nursing home admission and functional decline in elderly people: systematic review and meta-regression analysis. JAMA 2002;287(8):1022-8.

4. Meinck M, Lubke N, Lauterberg J, Robra BP. [Preventive home visits to the elderly: systematic review of available evidence]. Gesundheitswesen 2004;66(11):732-8.

5. Markle-Reid M, Browne G, Weir R, Gafni A, Roberts J, Henderson SR. The effectiveness and efficiency of home-based nursing health promotion for older people: a review of the literature. Med Care Res Rev 2006;63(5):531-69.

6. Elkan R, Kendrick D. What is the effectivess of home visiting or homebased support for older people? [http://www.euro.who.int/Document/e83105.pdf] 2004.

7. Ploeg J, Feightner J, Hutchison B, Patterson C, Sigouin C, Gauld M. Effectiveness of preventive primary care outreach interventions aimed at older people: meta-analysis of randomized controlled trials. Can Fam Physician 2005;51:1244-5.

8. van Rossum E, Frederiks CM, Philipsen H, Portengen K, Wiskerke J, Knipschild P. Effects of preventive home visits to elderly people. BMJ 1993;307(6895):27-32.

9. van Rossum E. Effects of preventive home visits to the elderly. PhD Thesis Maastricht University, 1993.

10. Nicolaides-Bouman A, van Rossum E, Kempen GI, Knipschild P. Effects of home visits by home nurses to elderly people with health problems: design of a randomised clinical trial in the Netherlands [ISRCTN92017183]. BMC Health Serv Res 2004;4(1):35.

11. Nicolaides-Bouman A, van Rossum E, Habets H, Kempen GI, Knipschild P. Home visiting programme for older people with health problems: process evaluation. J Adv Nurs 2007;58(5):425-35.

12. Bouman A, van Rossum E, Ambergen T, Kempen G, Knipschild P. Effects of a home visiting programme for older people with poor health status: a randomised clinical trial in the Netherlands. J Am Geriatr Soc 2008;56(3):397-404.

13. Bouman A, van Rossum E, Evers S, Ambergen T, Kempen G, Knipschild P. Effects on health care use and associated cost of a home visiting program for older people with a poor health status: a randomised clinical trial in the Netherlands. J Gerontol A Biol Sci Med Sci 2008;63:291-297.

14. Bula CJ, Berod AC, Stuck AE, Alessi CA, Aronow HU, Santos Eggimann B, et al. Effectiveness of preventive in-home geriatric assessment in well functioning, communitydwelling older people: secondary analysis of a randomized trial. J Am Geriatr Soc 1999;47(4):389-95.

15. Stuck AE, Minder CE, Peter Wuest I, Gillmann G, Egli C, Kesselring A, et al. A randomized trial of in-home visits for disability prevention in community-dwelling older people at low and high risk for nursing home admission. Archives of Internal Medicine 2000;160(7):97786.

16. Bernabei R, Landi F, Gambassi G, Sgadari A, Zuccala G, Mor V, et al. Randomised trial of impact of model of integrated care and case management for older people living in the community. BMJ Clin res ed 1998;316(7141):1348-51.

17. van Tulder MW, Assendelft WJ, Koes BW, Bouter LM. Method guidelines for systematic reviews in the Cochrane Collaboration Back Review Group for Spinal Disorders. Spine 1997;22(20):2323-30. 
18. van Tulder MW, Cherkin DC, Berman B, Lao L, Koes BW. The effectiveness of acupuncture in the management of acute and chronic low back pain. A systematic review within the framework of the Cochrane Collaboration Back Review Group. Spine 1999;24(11):1113-23.

19. van Hout HP, Nijpels G, van Marwijk HW, Jansen AP, Van't Veer PJ, Tybout W, et al. Design and pilot results of a single blind randomized controlled trial of systematic demandled home visits by nurses to frail elderly persons in primary care [ISRCTN05358495]. BMC Geriatr 2005;5:11.

20. Hall N. Randomized trial of a health promotion program for frail elders. Canadian Journal on Aging/La Revue Canadienne du vieillissement 1992;11(1):72-91.

21. Dalby DM, Sellors JW, Fraser FD, Fraser C, van Ineveld C, Howard M. Effect of preventive home visits by a nurse on the outcomes of frail elderly people in the community: a randomized controlled trial. Canadian Medical Association Journal / Journal de l'Association Medicale Canadienne 2000;162(4):497-500.

22. Yamada Y, Ikegami N. Preventive home visits for community-dwelling frail elderly people based on Minimum Data Set-Home Care: Randomized controlled trial. Geriatrics and Gerontology International 2003;3(4):236-242.

23. van Haastregt JC, Diederiks JP, van Rossum E, de Witte LP, Voorhoeve PM, Crebolder HF. Effects of a programme of multifactorial home visits on falls and mobility impairments in elderly people at risk: randomised controlled trial.[comment]. BMJ 2000;321(7267):994-8.

24. van Haastregt JC. Preventing falls and mobility impairments in elderly people living in the community. PhD thesis Maastricht University, 2002.

25. van Haastregt JC, van Rossum E, Diederiks JP, de Witte LP, Voorhoeve PM, Crebolder HF. Process-evaluation of a home visit programme to prevent falls and mobility impairments among elderly people at risk. Patient Educ Couns 2002;47(4):301-9.

26. Cochrane Handbook for Systematic Reviews of Interventions. Chicester: Wiley, 2005.

27. Williams EI, Greenwell J, Groom LM. The care of people over 75 years old after discharge from hospital: an evaluation of timetabled visiting by Health Visitor Assistants. J Public Health Med 1992;14(2):138-44.

28. Nikolaus T, Bach M. Preventing falls in community-dwelling frail older people using a home intervention team (HIT): results from the randomized Falls-HIT trial. J Am Geriatr Soc 2003;51(3):300-5.

29. Tinetti ME, Baker DI, McAvay G, Claus EB, Garrett P, Gottschalk M, et al. A multifactorial intervention to reduce the risk of falling among elderly people living in the community. $\mathrm{N}$ Engl J Med 1994;331(13):821-7.

30. Hansen FR, Spedtsberg K, Schroll M. Geriatric follow-up by home visits after discharge from hospital: a randomized controlled trial. Age and ageing 1992;21(6):445-50.

31. Markle-Reid M, Weir R, Browne G, Roberts J, Gafni A, Henderson S. Health promotion for frail older home care clients. J Adv Nurs 2006;54(3):381-95.

32. Pathy MS, Bayer A, Harding K, Dibble A. Randomised trial of case finding and surveillance of elderly people at home. Lancet 1992;340(8824):890-3.

33. Balaban DJ, Goldfarb NI, Perkel RL, Carlson BL. Follow-up study of an urban family medicine home visit program. J Fam Pract 1988;26(3):307-12.

34. Dunn RB, Lewis PA, Vetter NJ, Guy PM, Hardman CS, Jones RW. Health visitor intervention to reduce days of unplanned hospital re-admission in patients recently discharged from geriatric wards: the results of a randomised controlled study. Arch Gerontol Geriatr 1994;18(1):15-23.

35. Fletcher AE, Price GM, Ng ES, Stirling SL, Bulpitt CJ, Breeze E, et al. Population-based multidimensional assessment of older people in UK general practice: a cluster-randomised factorial trial. Lancet 2004;364(9446):1667-77.

36. Hebert R, Robichaud L, Roy PM, Bravo G, Voyer L. Efficacy of a nurse-led multidimensional preventive programme for older people at risk of functional decline. A randomized controlled trial. Age Ageing 2001;30(2):147-53.

37. Fried LP, Tangen CM, Walston J, Newman AB, Hirsch C, Gottdiener J, et al. Frailty in older adults: evidence for a phenotype. J Gerontol A Biol Sci Med Sci 2001;56(3):M146-56. 
38. Markle-Reid M, Browne G. Conceptualizations of frailty in relation to older adults. $\mathbf{J}$ Adv Nurs 2003;44(1):58-68.

39. Idler EL, Benyamini Y. Self-rated health and mortality: a review of twenty-seven community studies. J Health Soc Beh 1997;38(1):21-37.

40. Moher D, Schulz KF, Altman DG. The CONSORT statement: revised recommendations for improving the quality of reports of parallel-group randomized trials. Ann Intern Med 2001;134(8):657-62.

41. Oxman AD, Guyatt GH. A consumer's guide to subgroup analyses. Ann Intern Med 1992;116:78-84.

42. Pocock SJ, Hughes MD, Lee RJ. Statistical problems in the reporting of clinical trials. A survey of three medical journals. N Engl J Med 1987;317:426-32.

43. Stuck A, Kane RL. Whom Do Preventive Home Visits Help? J Am Geriatr Soc 2008;Editorial comments, 56(3):561-563.

44. Gill T, Baker DI, Gottschalk M, Gahbauer E, Charpentier P, de Regt P, et al. A prehabilitation program for physically frail community-living older persons. Arch Phys Med Rehabil 2003;84(3):394-404.

45. Phelan EA, Williams B, Penninx BW, LoGerfo JP, Leveille SG. Activities of daily living function and disability in older adults in a randomized trial of the health enhancement program. J Gerontol A Biol Sci Med Sci 2004;59(8):838-43.

46. Naylor MD, Brooten D, Campbell R, Jacobsen BS, Mezey MD, Pauly MV, et al. Comprehensive discharge planning and home follow-up of hospitalized elders: a randomized clinical trial. JAMA 1999;281(7):613-20.

47. Onder G, Liperoti R, Soldato M, Carpenter I, Steel K, Bernabei R, et al. Case management and risk of nursing home admission for older adults in home care: results of the AgeD in HOme Care Study. J Am Geriatr Soc 2007;55(3):439-44.

48. Luker K. Elderly women's opinions about the benefits of health visitor visits. Nursing Times 1981;77(12):suppl 9: 33-5.

49. Gunner Svensson F, Ipsen J, Olsen J, Waldstrom B. Prevention of relocation of the aged in nursing homes. Scandinavian journal of primary health care 1984;2(2):49-56.

50. Vetter NJ, Jones DA, Victor CR. Effect of health visitors working with elderly patients in general practice: a randomised controlled trial. BMJ Clin res ed 1984;288(6414):369-72.

51. Hendriksen C, Lund E, Stromgard E. Consequences of assessment and intervention among elderly people: a three year randomised controlled trial. BMJ Clin res ed 1984;289(6457):1522-4.

52. Sorensen KH, Sivertsen J. Follow-up three years after intervention to relieve unmet medical and social needs of old people. Comprehensive Gerontology. Section B, Behavioural, Social \& Applied Sciences 1988;2(2):85-91.

53. Carpenter GI, Demopoulos GR. Screening the elderly in the community: controlled trial of dependency surveillance using a questionnaire administered by volunteers. BMJ 1990;300(6734):1253-6.

54. McEwan RT, Davison N, Forster DP, Pearson P, Stirling E. Screening elderly people in primary care: a randomized controlled trial.[comment]. Brit J Gen Pract 1990;40(332):94-7.

55. Oktay JS, Volland PJ. Post-hospital support program for the frail elderly and their caregivers: a quasi-experimental evaluation. Am J Public Health 1990;80(1):39-46.

56. Clarke M, Clarke SJ, Jagger C. Social intervention and the elderly: a randomized controlled trial. Am J Epidemiol 1992;136(12):1517-23.

57. Vetter NJ, Lewis PA, Ford D. Can health visitors prevent fractures in elderly people? BMJ Clin res ed 1992;304(6831):888-90.

58. Fabacher D, Josephson K, Pietruszka F, Linderborn K, Morley JE, Rubenstein LZ. An inhome preventive assessment program for independent older adults: a randomized controlled trial. J Am Geriatr Soc 1994;42(6):630-8.

59. Wagner EH, LaCroix AZ, Grothaus L, Leveille SG, Hecht JA, Artz K, et al. Preventing disability and falls in older adults: a population-based randomized trial. Am J Publ Health 1994;84(11):1800-6. 
60. Archbold PG, Stewart BJ, Miller LL, Harvath TA, Greenlick MR, Van Buren L, et al. The PREP system of nursing interventions: a pilot test with families caring for older members. Preparedness (PR), enrichment (E) and predictability (P). Res Nurs Health 1995;18(1):3-16.

61. Stuck AE, Aronow HU, Steiner A, Alessi CA, Bula CJ, Gold MN, et al. A trial of annual inhome comprehensive geriatric assessments for elderly people living in the community.[comment]. N Engl J Med 1995;333(18):1184-9.

62. Hebert R, Robichaud L, Roy PM, Bravo G, Voyer L. Efficacy of a nurse-led multidimensional preventive programme for older people at risk of functional decline. A randomized controlled trial. Age and Ageing 2001;30(2):147-53.

63. Newbury JW, Marley JE, Beilby JJ. A randomised controlled trial of the outcome of health assessment of people aged 75 years and over. Medical Journal of Australia 2001;175(2):1047. 

7

General discussion 
In this thesis, a home visiting program conducted by home nurses for older persons with poor perceived health status is described, including the design of the study, a process evaluation of the intervention, and evaluations of the effects on health status, and on health care use and associated cost. Also, a systematic literature review is presented of randomized trials examining the effectiveness of intensive home visiting programs targeting older persons with poor health status. The objective of the program was to improve the functional abilities, perceived health and quality of life of the participants, compared with usual care. Reductions in institutionalization, especially hospital (re-) admissions, and total health care expenditures were also expected. This chapter discusses the methods and main findings of the study. Conclusions are formulated, the implications for health care practice are described and suggestions are given for further research.

\section{Main findings}

The home visits had no favorable effect on the health status of older persons with poor health at the end of the intervention (18 months) and after a 6-month follow-up period; this was observed for all primary outcome measures, e.g. selfrated health, functional status and quality of life. Minor observed improvements for several primary health outcomes after 12 months, in favor of the visited participants, were not statistically significant. The secondary outcomes also showed no favorable effects for the intervention group at the end of the intervention, e.g. depressive complaints, mental health, social support and loneliness. Also, no effect was found in favor of any of the pre-planned intervention subgroups at the end of the intervention period.

The effect evaluation on health care use revealed no positive effects of the home visiting program conducted by home nurses, with the exception of a higher distribution of aids and in-home modifications in favor of the intervention group. The health care items consisted of the number of admissions and the length of stay at the hospital, nursing home or home for older persons; contacts with medical specialists, general practitioners and paramedics; and hours of home care help. The referrals made by the nurses caused an expected increase in outpatient health care use, although these changes were not statistically significant and no impact on the use of institutional health care was observed. The overall cost of health care use per person, including the cost of the intervention program, was $€ 450$ higher in the intervention group than in the control group; the difference was not statistically significant. The results of the economic evaluation showed furthermore that the program had a low chance $(10 \%)$ of being cost-effective.

The process evaluation of the intervention program showed that the program was largely performed according to plan. Hundred and fifty-one of the 160 participants (95\%) received visits; 124 (78\%) received the complete program of 
eight visits over an 18-month period and 27 (17\%) received on average four visits. The program was feasible, and the participants and nurses appreciated it. Many health problems were detected and taken care of throughout the program, e.g. regarding social and psychological functioning, circulatory and musculoskeletal problems and vision or hearing impairments; on average, 10 problems and 11 interventions were recorded per participant. Overall, the participants complied with $61 \%$ of the nurses' recommendations.

The systematic review of trials on intensive home visiting programs for older persons with poor health status demonstrated that none of the trials of sufficient methodological quality, that is seven out of eight (including our trial), had a significant favorable effect for the main analysis of the home visit group compared with the usual care group on mortality, health status, service use or cost. The results of the review were in line with the results from the present trial.

\section{Methodological considerations}

We will discuss the methodology of the randomized trial and present possible explanations for the fact that the home visit intervention did not have the expected favorable outcome on health status and health care use.

\section{Selection of study population}

In a large part of the Sittard area, all community-living older persons aged 7084 received in 2002 a mailed questionnaire; this served as screening instrument and provided baseline measurements. The response rate was quite high $(76 \%)$ and when comparing the respondents with the total population there was no selective response for age and sex. From the respondents, one out of four persons (24\%) perceived their health as poor; this is consistent with national and regional figures. ${ }^{12}$

The participants were selected on poor self-rated health (a score of 1-5 on a scale of 1-10). Self-rated health has been reported to represent an overall measure for functional health abilities, including physical, mental and social functioning. $^{3}{ }^{4}$ The targeted population showed less-favorable scores on functional status and psychosocial functioning, and had a higher use of health services in comparison to the population of older persons with fair or good health status (a score of $\geq 6$ ); $93 \%$ of the older people with poor health status reported health problems at baseline. ${ }^{5}$ The relationship of (poor) self-rated health with various health domains was apparent for the selected target group.

We considered it important that the general practitioners (GPs) cooperated with the study. Two out of $21 \mathrm{GP}$ practices in the area did not want to cooperate and $139(28 \%)$ eligible persons were excluded. A relatively large number of older persons were registered in these two practices, because many older 
persons lived in the practices' vicinity. The GPs indicated that they could not manage a (possible) increase in consultations due to referrals made by the nurses. Based on the results from this study, however, the mean number of consultations and visits from the GP was only slightly higher in the intervention group compared to the control group. Still, there were sufficient eligible participants to raise the required number entering the study from 300 to 330 ; this slightly increased the power of the study. The number was not enough to include a third arm into the trial, even though this was not intended in the original design. For this group, home visits were planned to be carried out by volunteers (non-professionals). A comparison was therefore not possible between the effects of a 'sham-treatment' and the effects of visits by professional nurses. The recruitment procedure appeared adequate for the selection of the required study participants into the trial.

It is common practice in cost-effectiveness analyses that sample size calculations are based on effectiveness measures (in this study based on selfrated health scores from data of an earlier home visiting study in the Netherlands), ${ }^{3}$ and not on service use or cost measures. The latter have much higher coefficients of variation, and so generally require larger sample sizes. The study is therefore likely underpowered to detect health care use and cost differences; one has to be careful with the interpretation of these results.

\section{Drop-out}

At the end of the 24-month research period, 76 participants (23\%) had dropped out of the study. The reasons for drop-out, that is, mortality and self-withdrawal (mainly because of illness), were comparable for both study groups. The rates were somewhat higher in the intervention group compared to the control group. The small differences in mortality rates, $18 \%$ and $14 \%$, respectively, did not influence the results (unpublished data); the self-withdrawal rates were $10 \%$ and $5 \%$, respectively. For 37 participants (11\%) only the baseline measurements of the primary health outcomes were available and they were omitted from the analyses; within this subgroup there were no differences in health measures between the intervention and the control group (unpublished data). It is therefore unlikely that selective drop-out biased the results of the trial.

\section{Outcome measurements}

A questionnaire was mailed to the participants' home at the start of the trial and after 12,18 and 24 months of follow-up to obtain data for the primary outcome measures. The follow-up response rates were high (on average 95\%). Persons who had problems with administering the questionnaire were offered assistance; the volunteers who gave assistance were not aware of group assignment. Not being able to complete the questionnaires was not reported to be a reason for drop-out by the participants. A study by van Rossum, in which results obtained from personal interviews among community-living older persons were 
compared with those obtained from self-administered questionnaires, indicated that self-administered questionnaires seem an acceptable alternative to personal interviews among community-living older persons. ${ }^{3}$ The secondary outcome measures were collected after 18 months of follow-up through individual interviews, conducted by six trained, independent interviewers. Initially, they were blinded to group allocation; only at the end of the interview the group assignment was disclosed in order to ask the participants of the intervention group about their experiences with the home visits. The health care data over 24 months were mostly collected from computerized databases of medical administration offices; no information was supplied on group assignment. Overall, the data collection had a high degree of completeness; it is unlikely that possible inaccuracies biased the results.

To evaluate the effects on health status, mostly internationally accepted instruments were used. The Groningen Activity Restriction Scale (GARS), using a four-category response format, is a valid, reliable and detailed measure for assessing disability in older persons. ${ }^{6}$ The Medical Outcomes Study 20 -item Short Form Survey (SF-20) has been shown to correctly assess general health in an older population; the subscales on social and mental functioning were also shown to have sufficient psychometric properties in Dutch older persons. ${ }^{7}$

For two secondary health outcomes, cognitive impairments and social support, short-version scales were used (the MMSE-12 and SSL12-I, respectively). The aim was not to diagnose the older persons, which in general imply the use of elaborate indexes and trained observers, but to make a comparison between the study groups. The MMSE-12, which is simpler to use and takes less time than the MMSE-20, has shown to have diagnostic properties to screen for mild and severe cognitive impairments. ${ }^{8}$ The psychometric properties of the SSL12-I, a scale to measure social support, were found to be satisfactory in older persons, and to be especially useful when an indication of the amount of received social support is required. ${ }^{9}$ Overall, the used instruments seemed appropriate to measure potential benefits of the intervention.

\section{Confounding factors}

In order to enhance the comparability of the two groups at the start of the study, a stratified randomization procedure was used, based on three prognostic factors (functional status, health changes in the previous 3 months and contact with a medical specialist). The baseline characteristics were comparable for both groups, with the exception of 'health change in the 3 months before the start' (more favorable for the intervention group despite stratification), and 'influence on health status' (a higher percentage of intervention participants thought they had little influence on their health status). The last variable, together with other background characteristics and possible baseline differences in primary outcomes between the groups, were taken into account and adjusted for in our statistical analyses. The types of self-reported problems at baseline, classified 
according to ICPC-codes, were also similar between the groups (unpublished data). ${ }^{10}$ Other unknown or potential confounding factors were hopefully evenly distributed over the two groups after randomization.

\section{Intervention}

The intervention program consisted of eight home visits by a home nurse, over an 18-month period. The visits consisted of detecting problems and risks, followed by referrals, advice and giving information. A previous study in the Netherlands showed that positive effects of the visits for persons with poor health status emerged within 18 months; we therefore restricted the intervention to this period. The frequency of the visits was at the same time increased (bimonthly). Eighty-five percent of the participants were satisfied with the number of visits. The nurses reported that for some participants five or six visits may have been sufficient.

The nurses followed a structured protocol to conduct the visits, focusing on problems experienced by the older persons and those detected by the EasyCare Questionnaire. ${ }^{11}$ This elderly assessment questionnaire was chosen because it was easy to use, short, and highlighted issues related to functioning and quality of life for community-living individuals. Other assessment systems are available, e.g. the Resident Assessment Instrument Home Care (RAI-HC), but we considered this to be more time consuming and difficult in use for the professional level of the nurses. ${ }^{12}$ The protocol allowed the nurses freedom in choosing topics to be discussed during the visits. The amount of attention paid to a specific topic depended on the needs of the participants. The nurses considered the visiting protocol useful. A handbook of nursing diagnosis was used to standardize the formulation of problems, improving communication among nurses and researchers. ${ }^{13}$ The handbook also provided interventions to specific problems. By protocol, the number of problems to be managed per visit was limited to three, accompanied by maximum two interventions per problem (for feasibility reasons). This limitation did not seem to be a restriction, because the limit in number of problems and/or interventions was generally not reached.

Seventy-eight percent of participants completed the entire program of eight visits and $17 \%$ on average four. The number of withdrawals was low and often due to illness of the older persons. The participants were always visited by the same nurse. The home nurses were able to perform the visits without absence owing to illness or any other impediments. The process evaluation revealed that the home visits were largely performed according to protocol. There are no indications that control participants were exposed to similar interventions.

The nurses provided the participants with support, advice and information. In order to anticipate on the possibility of different approaches in carrying out the visits by the nurses, fortnightly meetings were held to discuss cases and common strategies were set out. After about five visits the type and number of referrals made by the nurses were investigated and found to be comparable; it is unlikely that differences in approach greatly influenced the results. 


\section{Discussion of main findings}

The effectiveness of the program may have been hampered by a number of factors. The nurses were not part of a multidisciplinary team, including geriatricians or physicians, limiting the medical component of the assessment, and this may have resulted in fewer recommendations. In this study, on average 11 recommendations (referrals and advice) were made to the intervention participants over, on average, seven visits per person. The number of recommendations is relatively high in comparison with a study by van Haastregt and colleagues, also targeting older persons with poor health (three recommendations over five visits, on average). ${ }^{14}$ This did not result, however, in more positive outcomes. In a trial by Stuck and colleagues, targeting the general population, 29 recommendations were made over, on average, 11 visits. ${ }^{1516}$ The large differences may be related to differences in the registration and definitions of recommendations, but may also be caused by the fact that in the study by Stuck and colleagues these were formulated in consultation with a geriatrician, resulting in more recommendations. Since not much detailed information is available from the intervention process in other home visiting trials targeting older persons with poor health, it is not possible to make further comparisons.

The compliance with the recommendations was reasonable. All activities were planned in agreement with the older persons to make them more motivated to become actively involved in handling the problems, and increasing the chances for the intervention to become effective. Of the intervention participants, 90\% reported that all important matters had been discussed and 93\% said that they could bring forward their own wishes. The adherence to recommendations was indeed higher than in the study by van Haastregt and colleagues $(61 \%$ compared to $46 \%) .{ }^{14}$ This may also have been caused by an extra telephone follow-up after the visits in the present study; possible barriers to comply with recommendations were discussed during the phone call. Compliance is a prerequisite for a successful outcome of the program and improvements are still possible. The nurses indicated that if, in their view, participants did not benefit from the program, the following factors may have played a role: these participants were less open to change, did not understand or had little knowledge about the relevant problems or were less motivated.

The nurses were experienced and motivated, although the lower professional level of the nurses may have been insufficient to effectively manage the many and complex problems that came forward throughout the intervention period. The first visit also turned out to be highly demanding for the nurses. In this light, it seems unlikely that non-professionals may have established positive effects.

The systematic review showed that intensive home visiting programs targeting older persons with poor health had no effect on their health status or service use; our trial-findings were supported by the methodologically sound included 
studies. This is also in line with subgroup analyses from a systematic review and meta-regression by Stuck and colleagues. ${ }^{17}$ Subgroup analyses of frail older persons in a review by Elkan and colleagues showed positive effects on mortality and nursing home admissions ${ }^{18}$, but these findings were based on four studies, including one non-randomized trial ${ }^{19}$ and one we scored as being of insufficient methodological quality. ${ }^{20}$ The results of studies not meeting our inclusion criteria (minimum four home visits a year and 12-months intervention period), but also reporting on the effects of home visiting programs for older persons with poor health, were mixed; the studies with positive outcomes showed benefits mostly for only one parameter, and we concluded that adding these studies would not have greatly influenced the findings of our review.

Not-yet published data from a Canadian trial by Brazil and colleagues of a home visiting program for frail older persons $(\mathrm{n}=719)$, showed furthermore no positive effects. $^{21} \mathrm{~A}$ recent publication of a large home-based geriatric care management program for low-income older persons $(n=951$, many with comorbid conditions) showed improvements in health-related quality of life, although most of the differences in outcomes between the intervention and control group involved social and mental health measures. ${ }^{22}{ }^{23}$ Functional status, measured by the SF-36, did not differ between the groups. For a predefined group in that study at high risk of hospitalization (112 intervention and 114 control), hospital admission rates were lower for the intervention group in the second year, but the differences in hospital days were not statistically different between the groups. Results from a study by Mahoney and colleagues, published in 2007, demonstrated that an in-home multifactor assessment and intervention, followed by 11 monthly telephone calls, for community-living older persons at risk for falls $(n=349)$, was not effective in decreasing falls. ${ }^{24}$ Also, no differences were found in admission rates to hospital and nursing homes, although a decrease was shown in nursing home days for the intervention group compared to the control group.

Recently published results from another trial in the Netherlands studying the effects of a problem-based geriatric intervention (on average, four visits in 3 months conducted by a geriatric specialist nurse) for frail older persons, showed improved functional abilities and mental well-being for the intervention group compared to the control group after 3 months of follow-up; the effect on functional abilities was no longer significant after 6 months. ${ }^{25}$ The sample size of the last trial was, however, small (85 intervention and 66 control). The results from a third trial conducted in the Netherlands are described in our systematic review; no beneficial effects of home visits to frail older persons were revealed. $^{26}$ 


\section{Conclusions}

The following conclusions on the effectiveness and feasibility of the conducted home visiting program can be drawn.

- The home visiting program, conducted by home nurses targeting older persons with poor health, aimed at improving their functional abilities, perceived health and quality of life, is not effective.

- The home visiting program does not reduce institutionalization.

- The health care cost of the persons receiving the home visits was higher than those for persons receiving usual care. The program has a low chance of being cost-effective.

- The home visiting program was feasible, the nurses and participants appreciated it, and the visits were implemented according to protocol.

- Our results are in line with the findings of other home visiting studies targeting older persons with poor health status; the programs appear not to be beneficial for this target population in the Netherlands or comparable health care settings in Western countries.

- This trial does not confirm the positive results from a subgroup analysis on the effects of home visits for older persons with poor health from an earlier Dutch study. Post hoc subgroup analyses should therefore be interpreted with caution.

\section{Implications for health care practice and further research}

The results of the randomized trial and the systematic review show that there are no arguments to add the home visiting program targeting older persons with poor health status to regular health care. In a number of countries, e.g. Japan, Denmark and Australia, preventive home visits are part of national policy and incorporated into regular health care for older persons. It seems essential, that the programs are judged on their merit again when targeting older persons in poor health. The United Kingdom withdrew this policy in 2004 based on the results of a large national trial, which showed that different forms of multidimensional assessment, targeting either the general population of older persons or frail older persons, offered almost no differences in patient outcomes. ${ }^{27}$

Before we started this study there was lack of clarity regarding the effective components of home visits, including which population the visiting programs should aim at. Our choice of target group was based on the results of a subgroup analysis from an earlier Dutch trial that revealed that the home visits seemed to be effective for older persons with poor health; ${ }^{3}$ a number of other studies also suggested that home visit programs should preferably be aimed at older persons at risk. ${ }^{20-30}$ The results of the included systematic review in this thesis and of subgroup analyses in a review by Stuck and colleagues showed that this choice 
does not appear to be a good one. ${ }^{17}$ On the other hand, a trial in the Netherlands showed that preventive home visits do not seem to be useful for the general population of older persons. ${ }^{31}$ The question remains whether these programs are effective for other target groups. Also, are there other types of programs that may be of benefit to older persons with a poor health status?

Stuck and Kane recently suggested that for older persons at low risk, some potential benefit may be expected from home visiting programs with a multidimensional approach, addressing the multiple potentially coexisting medical, functional, psychosocial, and environmental problems and risks of older persons. The programs should include long-term follow-up to allow for changes in response over time. ${ }^{32}$ There is good evidence, they further argue, that for persons at higher risk benefits may be expected from other types of interventions; e.g. favorable effects were shown by a 6-month intensive homebased physical therapy program (16 visits over 6 months $)^{33}$, a chronic disease self-management program including three visits and nine telephone calls ${ }^{34}$, and by a nurse-centered discharge plan with follow-up home visits for frail older persons discharged from hospital. ${ }^{35}$ The economic arguments for the last studies remain to be established however. The authors indicated that older persons at higher risk or those already disabled are likely to benefit from multidimensional interventions that target specific problems. ${ }^{32}$

Community care for older persons is the preferred option to hospital and long-term care, from a policy point-of-view, and also because the vast majority of persons wish to stay at home. ${ }^{36}$ Especially frail older persons are comfortable with home-based services. A multidimensional approach is recommended to address the medical, functional, psychosocial, and environmental problems of the older persons. This approach also reflects the perception of successful ageing of older adults, involving beliefs about physical, functional, psychological and social health. ${ }^{37}$ Collaboration between different professionals involved is necessary to manage the complex care that is often required. One of the responses in countries with a lack of collaboration between different care providers, has been the introduction of case (or care) management. ${ }^{36}$ In a large European study (11 countries) it was found that home care service based on a case management approach reduced risk of institutionalization in a population of frail older persons in home care. ${ }^{38}{ }^{39}$ Systematic care-coordination can be provided by a case-manager, but ideally health and social services should be more integrated and have coordination between the services to supply the best available options for the individual needs of older persons.

With the growth in the elderly population and a continuing increase in the incidence of chronic diseases, ${ }^{40}$ alternative strategies to improve the functional abilities and quality of life of community-living older persons should be developed and tested. A few positive interventions were already mentioned above. Telemedicine and other technologies also may be promising for the future of home care. Through the application of available consumer 
technologies 'connected health' is available to enable such services as remote monitoring, e.g. home-based management programs for chronic diseases, and activity monitoring, e.g. sensors recording vital signs and motor functions. Other technologies such as telephones, cellular phones and the internet have had an impact on home telehealth. By constantly being connected, patients are more involved in the decision-making process regarding their treatment and overall health management. ${ }^{41}$

Recently a literature review was published on the effects of telehomecare on older patients with chronic illness. ${ }^{42}$ Articles were selected if telehealth technology with peripheral medical devices (e.g. blood glucose monitor, stethoscope, blood pressure cuff) was used to deliver home care. Interventions that consisted of only telephone calls and did not involve video or in-person nurse contact were excluded. It was shown that patients and providers were accepting the technology and it appeared to have positive effects on outcomes such as self-management, adherence, general health, and length of hospital stay. Little impact on improvements in quality of life and function was identified. Telehomecare appeared to reduce health care cost. Bowles and Baugh noted however that, generally, studies had small sample sizes with diverse types and doses of telehomecare interventions for only a few chronic illnesses; most commonly heart failure, diabetes and chronic wounds. It was mentioned that telehomecare visits may be briefer and may allow for 15-20 video visits per day compared to 5-6 visits with traditional home care. Even though telehomecare has not had an impact on quality of life, it is cost-saving and improves several other outcomes. It enables the older persons with chronic diseases to stay at home and this may be an important argument to further explore and improve telehealth strategies.

Telesurveillance for community-living frail older persons (information was relayed between patient and a central call centre that coordinated services) has shown to substantially decrease the psychological burden of caregivers. ${ }^{43}$ No change was reported in the elders' quality of life and daily activity abilities, but the older persons were satisfied and the program was cost-saving, because of a reduction in hospital utilization and a decrease in home care visits. Caregivers have been reported to play an important role in the implementation of interventions for frail older persons; ${ }^{44}$ they also appear to benefit from technological support.

In a study by Rubinstein and colleagues targeting high-risk older persons, the intervention combined a structured telephone geriatric assessment, individualized referrals and recommendations, and ongoing telephone case management. ${ }^{45}$ The intervention could not achieve measurable improvement in functional status or hospitalization rates. Two studies included in the telehomecare review by Bowles and Baugh, reported that patients do not want to lose in-person contact completely. ${ }^{42}$ This may mean that a combination of telehomecare and in-person visits may be a necessary condition for such a program to become effective. Technology in itself may not be a guarantee for 
effectiveness and should not be seen as replacement of personal contacts and care; it may be supportive to in-person contacts. Although the effectiveness of various combinations of telehomecare and in-person visits is still uncertain, it seems a promising strategy.

The studies in this thesis show that the target group of older persons with poor health status may need other interventions to improve functional status and quality of life than the offered home visiting program. Further research is necessary to determine which strategies are most beneficial for them. Promising elements such as care-coordination, self-management of chronic diseases and/or other health problems, the integration of (medical) technology at older persons' homes in combination with in-person contacts, the targeting of specific problems, e.g. related to mobility and other ADLs, should be further explored. Research on new ways for improving compliance rates also deserves attention. Furthermore of interest to investigate are the effects of high-intensive programs, e.g. weekly home visits for older persons conducted by non-professional persons from voluntary home care organizations; these visits are occasionally performed in daily practice, but have not been examined yet. For a better comparison of different programs and to gain insight into the effective components of programs, a process evaluation should be included as part of trials. Cost-effectiveness analyses are necessary to present a business case over regular care. A better understanding of factors hampering and improving the effectiveness of home visiting programs for target groups other than those in good or poor health, and how these target groups should be defined, still stands as a relevant issue for future research. 


\section{References}

1. Giesbers H. Minder goede ervaren gezondheid 1995-1999.: Volksgezondheid Toekomst Verkenning, Nationaal Kompas Volksgezondheid. Bilthoven, RIVM, 2002.

2. Tijhuis M, Hoeymans N. Hoeveel mensen voelen zich niet gezond?: Volksgezondheid Toekomst Verkenning, Nationaal Kompas Volksgezondheid. Bilthoven, RIVM, 2002.

3. van Rossum E. Effects of preventive home visits to the elderly. PhD Thesis Maastricht University, 1993.

4. Idler EL, Benyamini Y. Self-rated health and mortality: a review of twenty-seven community studies. J Health Soc Beh 1997;38(1):21-37.

5. Nicolaides AIE, van Rossum E, Spaninks AM, Kempen GI, Heykens H, Houwen J, et al. De gezondheidstoestand van zelfstandig wonenende ouderen in Sittard en Geleen. Maastricht University, Gezondheidsstatistieken HQ 1064.L3, 2004.

6. Kempen GI, Miedema I, Ormel J, Molenaar W. The assessment of disability with the Groningen Activity Restriction Scale. Conceptual framework and psychometric properties. Soc Sci Med 1996;43(11):1601-10.

7. Kempen GI. Het meten van de gezondheidstoestand van ouderen. Een toepassing van een Nederlandse versie van de MOS-schaal. Tijdschrift voor Gerontologie en Geriatrie 1992;23(4):132-40.

8. Kempen GI, Brilman EI, Ormel J. De Mini-Mental State Examination: normeringsgegevens en een vergelijking van een 12- en 20-item versie in een steekproef ouderen uit de bevolking. Tijdschrift voor Gerontologie en Geriatrie 1995;26(4):163-72.

9. Kempen GI, van Eijk LM. The psychometric properties of the SSL12-I, a short scale for measuring social support in the elderly. Social Indicators Research 1995;35:302-12.

10. ICPC [http://www.hag.unimaas.nl/rnh/icpc.html].

11. Richardson J. The Easy-Care assessment system and its appropriateness for older people. Nurs Older People 2001;13(7):17-9.

12. Resident Assessment Instrument Home Care [http://www.interrai.org].

13. Carpenito L. Handbook of Nursing Diagnosis. 4th ed. Philadelphia: J.B. Lippincott Company, 1993.

14. van Haastregt JC, van Rossum E, Diederiks JP, de Witte LP, Voorhoeve PM, Crebolder HF. Process-evaluation of a home visit programme to prevent falls and mobility impairments among elderly people at risk. Patient Educ Couns 2002;47(4):301-9.

15. Stuck AE, Aronow HU, Steiner A, Alessi CA, Bula CJ, Gold MN, et al. A trial of annual inhome comprehensive geriatric assessments for elderly people living in the community.[comment]. N Engl J Med 1995;333(18):1184-9.

16. Alessi CA, Stuck AE, Aronow HU, Yuhas KE, Bula CJ, Madison R, et al. The process of care in preventive in-home comprehensive geriatric assessment. J Am Geriatr Soc 1997;45(9):1044-50.

17. Stuck AE, Egger M, Hammer A, Minder CE, Beck JC. Home visits to prevent nursing home admission and functional decline in elderly people: systematic review and meta-regression analysis. JAMA 2002;287(8):1022-8.

18. Elkan R, Kendrick D, Dewey M, Hewitt M, Robinson J, Blair M, et al. Effectiveness of home based support for older people: systematic review and meta-analysis. BMJ 2001;323(7315):719-25.

19. Williams EI, Greenwell J, Groom LM. The care of people over 75 years old after discharge from hospital: an evaluation of timetabled visiting by Health Visitor Assistants. J Public Health Med 1992;14(2):138-44.

20. Hall N. Randomized trial of a health promotion program for frail elders. Canadian Journal on Aging/La Revue Canadienne du vieillissement 1992;11(1):72-91.

21. Brazil K, Ploeg J, Hutchison B, Kaczorowski J, Goldsmith C, Dalby D, et al. The impact of preventive home visits to older adults at risk. Paper presented at the 60th Annual Scientific Meeting of the Gerontological Society of America, November 17, San Francisco. The Gerontologist 2007;47 (special issue II):84. 
22. Counsell SR, Callahan CM, Clark DO, Tu W, Buttar AB, Stump TE, et al. Geriatric care management for low-income seniors. JAMA 2007;298(22):2623-33.

23. Reuben DB. Better care for older people with chronic diseases. JAMA 2007;298(22):2673-4.

24. Mahoney JE, Shea TA, Przybelski R, Jaros L, Gangnon R, Cech S, et al. Kenosha County falls prevention study: a randomized, controlled trial of an intermediate-intensity, community-based multifactorial falls intervention. J Am Geriatr Soc 2007;55(4):489-98.

25. Melis R, van Eijken M, Teerenstra S. A randomised study of a multidisciplinary programme to intervene on geriatric syndromes in frail older people who live at home. J Gerontol A Biol Sci Med Sci 2008;63A(3):283-290.

26. van Hout HP, Nijpels G, van Marwijk HW, Jansen AP, Van't Veer PJ, Tybout W, et al. Design and pilot results of a single blind randomized controlled trial of systematic demandled home visits by nurses to frail elderly persons in primary care [ISRCTN05358495]. BMC Geriatr 2005;5:11.

27. Fletcher AE, Price GM, Ng ES, Stirling SL, Bulpitt CJ, Breeze E, et al. Population-based multidimensional assessment of older people in UK general practice: a cluster-randomised factorial trial. Lancet 2004;364(9446):1667-77.

28. Pathy MS, Bayer A, Harding K, Dibble A. Randomised trial of case finding and surveillance of elderly people at home. Lancet 1992;340(8824):890-3.

29. Leveille SG, Wagner EH, Davis C, Grothaus L, Wallace J, LoGerfo M, et al. Preventing disability and managing chronic illness in frail older adults: a randomized trial of a community-based partnership with primary care.[comment]. J Am Geriatr Soc 1998;46(10):1191-8.

30. Robichaud L, Hébert R, Roy PM, Roy C. A preventive program for community-dwelling elderly at risk of functional decline: a pilot study. Arch Gerontol Geriatr 2000;30:73-84.

31. van Rossum E, Frederiks CM, Philipsen H, Portengen K, Wiskerke J, Knipschild P. Effects of preventive home visits to elderly people. BMJ 1993;307(6895):27-32.

32. Stuck A, Kane RL. Whom Do Preventive Home Visits Help? J Am Geriatr Soc 2008;Editorial comments, 56(3):561-563.

33. Gill TM, Baker DI, Gottschalk M, Peduzzi PN, Allore H, Byers A. A program to prevent functional decline in physically frail, elderly persons who live at home. $\mathrm{N}$ Engl $\mathrm{J}$ Med 2002;347(14):1068-74.

34. Phelan EA, Williams B, Penninx BW, LoGerfo JP, Leveille SG. Activities of daily living function and disability in older adults in a randomized trial of the health enhancement program. J Gerontol A Biol Sci Med Sci 2004;59(8):838-43.

35. Naylor MD, Brooten D, Campbell R, Jacobsen BS, Mezey MD, Pauly MV, et al. Comprehensive discharge planning and home follow-up of hospitalized elders: a randomized clinical trial. JAMA 1999;281(7):613-20.

36. Smith R. Improving the management of chronic disease. BMJ 2003;327(7405):12.

37. Phelan EA, Anderson LA, LaCroix AZ, Larson EB. Older adults' views of 'successful aging'-how do they compare with researchers' definitions? J Am Geriatr Soc 2004;52(2):211-6.

38. Onder G, Liperoti R, Soldato M, Carpenter I, Steel K, Bernabei R, et al. Case management and risk of nursing home admission for older adults in home care: results of the AgeD in HOme Care Study. J Am Geriatr Soc 2007;55(3):439-44.

39. Carpenter I, Gambassi G, Topinkova E, Schroll M, Finne-Soveri H, Henrard JC, et al. Community care in Europe. The Aged in Home Care project (AdHOC). Aging Clin Exp Res 2004;16(4):259-69.

40. Barondess JA. The shape of things to come: homage to H.G. Wells. J Am Geriatr Soc 2007;55:1877-1881.

41. Wootton R, Dimmick SL, Kvedar JC. Home telehealth: connecting care within the community: RSM Press telemedicine series, 2006.

42. Bowles KH, Baugh AC. Applying research evidence to optimize telehomecare. J Cardiovasc Nurs 2007;22(1):5-15. 
43. Vincent C, Reinharz D, Deaudelin I, Garceau M, Talbot LR. Public telesurveillance service for frail elderly living at home, outcomes and cost evolution: a quasi experimental design with two follow-ups. Health Qual Life Outcomes 2006;4:41.

44. van Eijken M. Community care for older people living at home. PhD Thesis Radboud University Nijmegen, 2007.

45. Rubenstein LZ, Alessi CA, Josephson KR, Trinidad Hoyl M, Harker JO, Pietruszka FM. A randomized trial of a screening, case finding, and referral system for older veterans in primary care. J Am Geriatr Soc 2007;55(2):166-74. 



\title{
Appendix
}

\section{De gezondheidstoestand van zelfstandig wonende ouderen: resultaten van een schriftelijke enquête in Sittard en Geleen}

\author{
The health status of community-living \\ elderly: results of a postal questionnaire \\ in the south of the Netherlands
}

\begin{abstract}
Ans Bouman ${ }^{1}$, Erik van Rossum ${ }^{1}$, Annemarie Spaninks ${ }^{1}$, Gertrudis Kempen ${ }^{2}$ and Paul Knipschild ${ }^{3}$
\end{abstract}

Dit artikel is een verkorte versie van een elektronisch verschenen rapport.

This article is an abbreviated version of an electronically available manuscript.

AIE Nicolaides, E v Rossum, AM Spaninks, GIJM Kempen, H Heykens, J Houwen, Y Monse, PG Knipschild. De gezondheidstoestand van zelfstandig wonende ouderen in Sittard en Geleen. Verslag van een schriftelijke enquête naar de zelfredzaamheid, het psychosociaal functioneren, het zorggebruik en de gezondheidsbeleving.

Maastricht University 2004, Health Statistics HQ 1064.L3

1 Department of Epidemiology, Maastricht University, Maastricht, the Netherlands

2 Department of Health Care Studies, Maastricht University, Maastricht, the Netherlands

3 Department of General Practice, Maastricht University, Maastricht, the Netherlands 


\section{Samenvatting}

In dit artikel worden de resultaten gepresenteerd van een schriftelijke enquête naar de gezondheidstoestand van thuiswonende ouderen (70-84 jaar, $n=4,901)$ in Sittard en omgeving. In de enquête stond de zelfredzaamheid, het psychosociaal functioneren, het zorggebruik en de gezondheidsbeleving centraal. De respons bedroeg $76 \%$.

Ongeveer 10\% van de ouderen is ADL-afhankelijk en bijna $40 \%$ kan tenminste een huishoudelijke (HDL) activiteit niet meer zelfstandig uitvoeren. Vrouwen zijn vaker dan mannen afhankelijk bij ADL-activiteiten en mannen vaker bij HDL-activiteiten.

Op psychosociaal functioneren scoren vrouwen iets minder goed dan mannen. Ouderen scoren aanmerkelijk slechter naarmate men zich meer eenzaam voelt. Bijna $70 \%$ van de ouderen heeft contact gehad met de huisarts in de 3 maanden voorafgaand aan de enquête en $46 \%$ bezocht de specialist. Eén op de vier ouderen maakt gebruik van thuiszorg, vooral huishoudelijke hulp en $85 \%$ rapporteert bij ziekte te kunnen terugvallen op mantelzorg. Een kwart geeft zich een onvoldoende voor de gezondheid. Deze ouderen rapporteren een slechtere zelfredzaamheid, hebben meer psychosociale problemen en gebruiken meer professionele zorg.

Een postenquête lijkt een goed instrument om gezondheidsgegevens over ouderen te verzamelen. De bevindingen in Sittard en omgeving zijn in overeenstemming met die in andere steden. Er zijn aanwijzingen dat de zelfredzaamheid onder thuiswonende ouderen in de afgelopen 15 jaar is verbeterd.

\section{Summary}

This article reports the results of a postal survey on the health status of communitydwelling elderly $(70-84$ yrs, $n=4,901)$ in a southern region of the Netherlands. The questionnaire dealt with the functional status, psychosocial functioning, service use and self-rated health of the elderly. The response rate was $76 \%$.

Approximately $10 \%$ of the population had at least one activity of daily living (ADL) dependency and nearly $40 \%$ had at least one dependency in performing instrumental (household, IADL) activities. Women scored worse on ADLs and men on IADLs. Psychosocial functioning is lower among women compared to men. Elderly people score much lower on psychosocial functioning if they feel lonelier. Almost $70 \%$ consulted their general practitioner in the previous 3 months, and $46 \%$ a medical specialist. One out of four elderly people used professional home care (mainly domestic help) and $85 \%$ reported the availability of informal care, if needed.

Twenty-four percent of the population rated their health as poor. This subgroup scored worse on functional status and psychosocial functioning, and their service use was higher.

A postal survey appears a useful instrument to gather health data among elderly people. The results are in line with those reported for other Dutch cities. There are indications that the functional status of community-living elderly has improved over the last 15 years. 


\section{Inleiding}

In de afgelopen 20 jaar is veel informatie voorhanden gekomen over de gezondheidstoestand, de zorgbehoeften en het zorggebruik van thuiswonende ouderen. Deze informatie is deels afkomstig uit regelmatig monitoren van deze groep met gezondheidsenquêtes door GGD-en (regionaal of provinciaal) of het CBS. ${ }^{1}$ Incidenteel vindt vanuit de GGD aanvullend onderzoek plaats, waarin bijvoorbeeld de oudere leeftijdsgroepen (75-plussers) expliciet worden betrokken. ${ }^{2}$ In het regulier monitoren ontbreken deze oudere groepen veelal. Daarnaast zijn er vanaf 1990 een aantal grootschalige cohortstudies onder thuiswonende ouderen gestart, die eveneens veel informatie hebben gegenereerd. Bekende voorbeelden van dergelijke studies zijn de Longitudinal Aging Study Amsterdam (LASA, $\mathrm{n}=3107,55-85$ jaar), de Groningen Longitudinal Aging Study (GLAS, $\mathrm{n}=5279,57$ jaar en ouder), het Erasmus Rotterdam Gezondheid en Ouderen onderzoek (ERGO, n=7983, 55 jaar en ouder) en de Zutphen Ouderen Studie ( $n=487,70-90$ jarige mannen). ${ }^{3-6}$

In het kader van een gecontroleerd experiment naar de effecten van huisbezoeken vanuit de thuiszorg aan zelfstandig wonende ouderen met (ervaren) gezondheidsproblemen is eind 2002 een schriftelijke enquête uitgevoerd bij een grote groep 70-84 jarigen in de regio Sittard. ${ }^{7}$ De enquête was gericht op de zelfredzaamheid, het psychosociaal functioneren, het zorggebruik en de gezondheidsbeleving van deze ouderen. De verkregen gegevens zijn geschikt om een beeld te schetsen van de gezondheidstoestand van een grote groep ouderen. Naast inzicht in de prevalentie van beperkingen en klachten was het doel van deze studie om de relaties tussen de onderzochte gezondheidsdomeinen te bestuderen en om te kijken of er over een periode van ruim tien jaar veranderingen in de gezondheidstoestand van ouderen in de onderzochte regio waren opgetreden. Dit laatste was mogelijk doordat in 1990 een vergelijkbare enquête bij ouderen in Sittard is uitgevoerd. ${ }^{8}$

\section{Methode}

\section{Populatie en meetinstrument}

De vragenlijst is toegezonden aan 4901 zelfstandige wonende ouderen in een groot deel van Sittard, het noordelijke deel van Geleen, en Munstergeleen. De lijst bevatte 46 vragen, onderverdeeld in vijf secties. Naast achtergrondkenmerken als woonvorm en opleiding, zijn vragen gesteld over de zelfredzaamheid, het psychosociaal functioneren, het zorggebruik en de gezondheidsbeleving. 


\section{Zelfredzaamheid}

De zelfredzaamheid is gemeten met de Groningen Activiteiten Restrictie Schaal (GARS). ${ }^{9} \quad$ Deze omvat twee subschalen: algemene dagelijkse levensverrichtingen (ADL, 11 items) en huishoudelijke dagelijkse levensverrichtingen (HDL, zeven items). Op een 4-puntsschaal kan de respondent per activiteit aangeven of deze zelfstandig zonder problemen uitgevoerd kan worden (score '1'), met enige moeite (score '2'), met veel moeite (score '3'), of niet zelfstandig (score '4'). Op basis van de antwoorden zijn twee somscores berekend: een score zonder dichotomiesering van de antwoordcategorieën (range 18-72) en een somscore voor het aantal activiteiten dat al dan niet zelfstandig uitgevoerd kan worden (score 1-3 versus score 4, range 0-18).

\section{Psychosociaal functioneren}

Voor het meten van de psychische gezondheid en het sociaal functioneren (beperkingen in sociale activiteiten om gezondheidsredenen) zijn twee subschalen van de MOS Short-Form General Health Survey (SF-20) gebruikt. ${ }^{10}$ De subschaal psychische gezondheid bestaat uit vijf items en sociaal functioneren uit een item (alle met zes antwoordmogelijkheden die een gradatie in ernst aangeven). De scores op de items worden gesommeerd en getransformeerd naar een schaal van 0 tot $100 .{ }^{11}$

Omdat de subschaal sociaal functioneren uit slechts een item bestaat, zijn twee vragen over de gemiddelde bezoekfrequentie per week toegevoegd om een beter zicht te krijgen op het sociaal functioneren (op bezoek gaan en bezoek ontvangen). Daarnaast is een vraag over eenzaamheidsgevoelens in de afgelopen maand toegevoegd (zes antwoordmogelijkheden).

\section{Zorggebruik}

Om een indicatie te krijgen van het zorggebruik is aan de ouderen gevraagd, of men in de 3 maanden voorafgaand aan het invullen van de vragenlijst, contact heeft gehad met de huisarts of specialist(en) en eventuele ziekenhuisopname(s) heeft gehad. Ook is gevraagd naar het gebruik van thuiszorg (wijkverpleegkundige en huishoudelijke hulp), het op een wachtlijst staan voor opname in een verzorgingshuis of verpleeghuis, en de beschikbaarheid van mantelzorg.

\section{Gezondheidsbeleving}

De gezondheidsbeleving is gemeten met een rapportcijfer (1-10 punten) voor de eigen gezondheid. Tevens is gevraagd hoe men de gezondheid in vergelijking met 3 maanden geleden ervaart en in hoeverre men zelf invloed kan uitoefenen op de gezondheidssituatie. 


\section{Uitvoering van de enquête}

De enquête is eind 2002 uitgevoerd. Bij de vragenlijst waren een aanbevelingsbrief van de gemeente, een informatiebrief van de onderzoekers en een portovrije antwoordenvelop, bijgevoegd. Indien de vragenlijst na circa 2 weken niet terugontvangen was, werd een schriftelijke herinnering gestuurd.

Ondersteuning bij het invullen van de lijst kon gevraagd worden aan een vrijwilliger van de ouderenbond. Per wijk was een vrijwilliger beschikbaar om de ouderen telefonisch te woord te staan of om eventueel thuis op bezoek te gaan. Aan het project is op diverse wijzen aandacht besteed in de lokale media (dagbladen, lokale televisie en radio).

Zeer onvolledig ingevulde vragenlijsten werden als niet bruikbaar beschouwd. Dit betrof 18 lijsten. Personen die inmiddels verhuisd waren naar een plaats buiten het onderzoeksgebied, of opgenomen waren in een verzorgingshuis of verpleeghuis, werden niet in het bestand opgenomen. In een aantal gevallen werd telefonisch of schriftelijk contact opgenomen met de oudere. Dit gebeurde vooral indien antwoorden onduidelijk waren of belangrijke vragen met het oog op het vervolgonderzoek niet ingevuld waren.

\section{Data-analyse}

Waar mogelijk, zijn ontbrekende scores op items van een (sub)schaal gesubstitueerd door de gemiddelde score op de overige items. Per schaal werd een maximum gesteld aan ontbrekende scores: voor de GARS totaalscore en de ADL-subschaal twee items, voor de HDL-subschaal een item en voor psychische gezondheid twee items. In alle andere gevallen werd een score als 'missing' beschouwd.

Met het oog op het beschrijvende karakter van dit onderzoek zijn de gegevens op eenvoudige wijze geanalyseerd (frequentieverdelingen en gemiddelde scores en standaarddeviaties (SD)). Verbanden tussen variabelen zijn, afhankelijk van het meetniveau, statistisch getoetst met behulp van gebruikelijke toetsen (Chikwadraattoets, t-toets of variatieanalyse (ANOVA)). Met behulp van meervoudige lineaire regressie (voorwaartse stapsgewijze procedure) is gekeken naar de invloed van verschillende variabelen op gezondheidsbeleving.

\section{Resultaten}

Bij de presentatie van de bevindingen wordt achtereenvolgens ingegaan op de respons en achtergrondkenmerken van de respondenten, de zelfredzaamheid, het psychosociaal functioneren, het zorggebruik en de gezondheidsbeleving van de ouderen. 


\section{Respons en kenmerken populatie}

Van de 4901 verstuurde vragenlijsten zijn 3671 bruikbare lijsten geretourneerd. Rekening houdend met sterfte en opnames ten tijde van de enquête $(n=51)$ bedraagt de respons $76 \%$. De gemiddelde leeftijd van de respondenten is $75.5 \pm$ 3.9 en deze verschilt nauwelijks tussen mannen en vrouwen. Bijna de helft van de respondenten is tussen de 70 en 74 jaar en $58 \%$ vrouw. Naar leeftijd en geslacht komen de respondenten goed overeen met de totaal aangeschreven populatie (zie Tabel 1).

\begin{tabular}{cccccc}
$\begin{array}{c}\text { Tabel 1 } \\
\text { Table 1 }\end{array}$ & $\begin{array}{c}\text { Respondenten en totale populatie naar leeftijd en geslacht } \\
\text { Respondents and total population according to age and sex }\end{array}$ \\
\hline \multirow{6}{*}{ Geslacht } & man & 1537 & $(42 \%)$ & $\begin{array}{r}\text { Respondenten } \\
(\mathrm{n}=3671)\end{array}$ & $\begin{array}{c}\text { Totale populatie } \\
(\mathrm{n}=4901)\end{array}$ \\
& vrouw & 2152 & $(58 \%)$ & 2046 & $(42 \%)$ \\
\multirow{2}{*}{ Leeftijd } & $70-74$ & 1679 & $(46 \%)$ & 2212 & $(45 \%)$ \\
& $75-79$ & 1296 & $(35 \%)$ & 1702 & $(35 \%)$ \\
& $80-84$ & 714 & $(19 \%)$ & 987 & $(20 \%)$ \\
\hline
\end{tabular}

Bijna 40 procent van de respondenten woont alleen en het merendeel van de alleenstaanden is vrouw (80\%). Zoals verwacht stijgt het aantal alleenstaanden met de leeftijd. Over het algemeen zijn de vrouwelijke respondenten lager opgeleid dan de mannelijke: $85 \%$ van de vrouwen heeft een laag opleidingsniveau (hoogstens lagere school of afgerond lager beroepsonderwijs), tegen $60 \%$ van de mannen.

\section{Zelfredzaamheid}

Tabel 2 en 3 geven, voor respectievelijk de ADL- en HDL-activiteiten, het percentage ouderen aan dat een activiteit niet zelfstandig kan uitvoeren (score 4). De zelfredzaamheid ten aanzien van ADL-activiteiten is beduidend groter in vergelijking met de HDL-activiteiten. Naarmate de leeftijd stijgt, neemt de ADL- en HDL-afhankelijkheid toe. Dit geldt zowel voor mannen als voor vrouwen. 
Tabel 2 Percentage ADL-afhankelijkheid naar leeftijd en geslacht

Table 2 Percentage of ADL-dependency according to age and sex

\begin{tabular}{|c|c|c|c|c|c|c|}
\hline & \multirow[b]{2}{*}{ leeftijd } & \multicolumn{5}{|c|}{ ADL-afhankelijkheid* } \\
\hline & & $\begin{array}{c}\text { mannen } \\
(\%)\end{array}$ & $\begin{array}{c}\text { totaal } \\
(\%)\end{array}$ & $\begin{array}{c}\text { vrouwen } \\
(\%)\end{array}$ & $\begin{array}{c}\text { totaal } \\
(\%)\end{array}$ & $\begin{array}{c}\text { TOTAAL } \\
(\%)\end{array}$ \\
\hline \multirow{3}{*}{$\begin{array}{l}\text { aan- en } \\
\text { uitkleden }\end{array}$} & $70-74$ & 1.5 & & 1.5 & & \\
\hline & $75-79$ & 2.6 & 2.4 & 2.4 & 2.1 & 2.2 \\
\hline & $80-84$ & 2.4 & & 2.7 & & \\
\hline \multirow{3}{*}{$\begin{array}{l}\text { in en uit bed } \\
\text { komen }\end{array}$} & $70-74$ & 1.0 & & 0.6 & & \\
\hline & $75-79$ & 1.1 & 1.1 & 0.8 & 0.7 & 0.8 \\
\hline & $80-84$ & 1.5 & & 0.5 & & \\
\hline \multirow{3}{*}{$\begin{array}{l}\text { vanuit stoel } \\
\text { overeind } \\
\text { komen }\end{array}$} & $70-74$ & 0.8 & & 0.6 & & \\
\hline & $75-79$ & 0.4 & 0.7 & 0.4 & 0.4 & 0.5 \\
\hline & $80-84$ & 1.1 & & 0.0 & & \\
\hline \multirow{3}{*}{$\begin{array}{l}\text { gezicht en } \\
\text { handen } \\
\text { wassen }\end{array}$} & $70-74$ & 1.0 & & 0.4 & & \\
\hline & $75-79$ & 0.4 & 0.9 & 0.7 & 0.5 & 0.7 \\
\hline & $80-84$ & 1.5 & & 0.4 & & \\
\hline \multirow{3}{*}{$\begin{array}{l}\text { Zichzelf } \\
\text { wassen }\end{array}$} & $70-74$ & 2.5 & & 2.7 & & \\
\hline & $75-79$ & 4.3 & 3.7 & 6.3 & 5.7 & 4.9 \\
\hline & $80-84$ & 6.1 & & 11.3 & & \\
\hline \multirow{3}{*}{$\begin{array}{l}\text { naar het toilet } \\
\text { gaan }\end{array}$} & $70-74$ & 0.8 & & 0.3 & & \\
\hline & $75-79$ & 1.1 & 0.9 & 0.5 & 0.3 & 0.6 \\
\hline & $80-84$ & 0.8 & & 0.0 & & \\
\hline \multirow{3}{*}{$\begin{array}{l}\text { eten en } \\
\text { drinken }\end{array}$} & $70-74$ & 0.4 & & 0.2 & & \\
\hline & $75-79$ & 0.0 & 0.3 & 0.4 & 0.2 & 0.3 \\
\hline & $80-84$ & 0.8 & & 0.0 & & \\
\hline \multirow{3}{*}{$\begin{array}{l}\text { rondlopen in } \\
\text { huis }\end{array}$} & $70-74$ & 1.0 & & 0.4 & & \\
\hline & $75-79$ & 0.6 & 0.9 & 0.4 & 0.6 & 0.7 \\
\hline & $80-84$ & 1.1 & & 1.4 & & \\
\hline \multirow[t]{3}{*}{ trappen lopen } & $70-74$ & 2.6 & & 3.9 & & \\
\hline & $75-79$ & 4.0 & 3.6 & 6.1 & 6.3 & 5.2 \\
\hline & $80-84$ & 5.8 & & 11.8 & & \\
\hline \multirow{3}{*}{$\begin{array}{l}\text { buitenshuis } \\
\text { lopen }\end{array}$} & $70-74$ & 2.1 & & 2.1 & & \\
\hline & $75-79$ & 3.2 & 2.5 & 4.2 & 3.8 & 3.3 \\
\hline & $80-84$ & 2.3 & & 6.6 & & \\
\hline \multirow{3}{*}{$\begin{array}{l}\text { voeten en } \\
\text { teennagels } \\
\text { verzorgen }\end{array}$} & $70-74$ & 12.0 & & 25.6 & & \\
\hline & $75-79$ & 20.5 & 18.5 & 38.2 & 35.8 & 28.6 \\
\hline & $80-84$ & 32.6 & & 53.3 & & \\
\hline
\end{tabular}

* Percentage ouderen dat activiteit alleen met hulp van anderen kan uitvoeren. 
Tabel 3 Percentage HDL-afhankelijkheid naar leeftijd en geslacht Table 3 Percentage of IADL-dependency according to age and sex

\begin{tabular}{|c|c|c|c|c|c|c|}
\hline & \multirow[b]{2}{*}{ leeftijd } & \multicolumn{5}{|c|}{ HDL-afhankelijkheid* } \\
\hline & & $\begin{array}{c}\text { mannen } \\
(\%)\end{array}$ & $\begin{array}{c}\text { totaal } \\
(\%)\end{array}$ & $\begin{array}{c}\text { vrouwen } \\
(\%)\end{array}$ & $\begin{array}{c}\text { totaal } \\
(\%)\end{array}$ & $\begin{array}{c}\text { TOTAAL } \\
(\%)\end{array}$ \\
\hline \multirow{3}{*}{$\begin{array}{l}\text { ontbijt en lunch } \\
\text { klaarmaken }\end{array}$} & $70-74$ & 2.9 & & 1.3 & & \\
\hline & $75-79$ & 5.2 & 4.3 & 1.9 & 1.6 & 2.7 \\
\hline & $80-84$ & 6.6 & & 2.0 & & \\
\hline \multirow{3}{*}{$\begin{array}{l}\text { warme maaltijd } \\
\text { klaarmaken }\end{array}$} & $70-74$ & 12.3 & & 2.3 & & \\
\hline & $75-79$ & 20.3 & 17.8 & 4.4 & 4.2 & 9.8 \\
\hline & $80-84$ & 28.2 & & 7.7 & & \\
\hline \multirow{3}{*}{$\begin{array}{l}\text { licht huishoudelijk } \\
\text { werk }\end{array}$} & $70-74$ & 5.3 & & 3.1 & & \\
\hline & $75-79$ & 9.1 & 7.5 & 5.3 & 5.3 & 6.2 \\
\hline & $80-84$ & 10.8 & & 10.0 & & \\
\hline \multirow{3}{*}{$\begin{array}{l}\text { zwaar } \\
\text { huishoudelijk } \\
\text { werk }\end{array}$} & $70-74$ & 11.7 & & 21.8 & & \\
\hline & $75-79$ & 20.5 & 18.8 & 36.0 & 32.7 & 27.0 \\
\hline & $80-84$ & 35.7 & & 50.1 & & \\
\hline \multirow[t]{3}{*}{ kleren wassen } & $70-74$ & 29.3 & & 5.7 & & \\
\hline & $75-79$ & 41.5 & 37.7 & 10.3 & 9.8 & 20.8 \\
\hline & $80-84$ & 54.0 & & 17.6 & & \\
\hline \multirow[t]{3}{*}{ bed verschonen } & $70-74$ & 17.8 & & 13.5 & & \\
\hline & $75-79$ & 28.5 & 25.0 & 19.3 & 18.6 & 21.1 \\
\hline & $80-84$ & 38.1 & & 27.9 & & \\
\hline \multirow{3}{*}{$\begin{array}{l}\text { boodschappen } \\
\text { doen }\end{array}$} & $70-74$ & 6.8 & & 13.6 & & \\
\hline & $75-79$ & 12.2 & 10.8 & 21.4 & 0.6 & 16.6 \\
\hline & $80-84$ & 19.4 & & 34.1 & & \\
\hline
\end{tabular}

* Percentage ouderen dat activiteit alleen met hulp van anderen kan uitvoeren.

Verschillen tussen mannen en vrouwen in het uitvoeren van ADL-activiteiten komen vooral naar voren bij het zichzelf kunnen wassen, trappen lopen, buitenshuis lopen, en voeten en teennagels verzorgen; vrouwen ondervinden hier vaker beperkingen dan mannen. Bij de HDL-activiteiten zien we juist dat mannen vaker beperkingen ondervinden, behalve wanneer het gaat om zwaar huishoudelijk werk en boodschappen doen. De overige HDL-activiteiten zijn activiteiten die, traditioneel gezien, vaker door vrouwen worden uitgevoerd. Het 
zwaar huishoudelijke werk levert de meeste problemen op: een op de vier ouderen is hierbij aangewezen op hulp.

Voor elke ouder is een optelsom gemaakt van het aantal activiteiten dat men niet meer zelfstandig kan uitvoeren (score 4). Bij de optelsom is het item 'voeten en teennagels verzorgen' bij de ADL-activiteiten achterwege gelaten, omdat dit item niet van grote invloed is op de dagelijkse zelfzorg. Ongeveer 90 procent van de ouderen heeft geen enkele ADL-afhankelijkheid en ongeveer 60 procent geen enkele HDL-afhankelijkheid (tabel 4). Ruim een op de zes ouderen is minstens voor drie HDL-activiteiten afhankelijk van hulp van anderen.

\section{Tabel 4 Procentuele verdeling van het aantal niet zelfstandig uit te voeren ADL- en HDL-activiteiten naar geslacht}

Table 4 Percentage distribution of the number of ADL and IADLdependencies according to sex

\begin{tabular}{|c|c|c|c|c|c|c|}
\hline \multirow{2}{*}{$\begin{array}{l}\text { Afhankelijkheid } \\
\text { activiteiten } \\
\text { (aantal) }\end{array}$} & \multicolumn{3}{|c|}{ ADL } & \multicolumn{3}{|c|}{ HDL } \\
\hline & $\begin{array}{c}\operatorname{man} \\
(\%)\end{array}$ & $\begin{array}{c}\text { vrouw } \\
(\%)\end{array}$ & $\begin{array}{c}\text { totaal } \\
(\%)\end{array}$ & $\begin{array}{c}\operatorname{man} \\
(\%)\end{array}$ & $\begin{array}{c}\text { vrouw } \\
(\%)\end{array}$ & $\begin{array}{c}\text { totaal } \\
(\%)\end{array}$ \\
\hline 0 & 94.2 & 89.3 & 91.3 & 59.0 & 63.0 & 61.3 \\
\hline $1-2$ & 3.5 & 8.4 & 6.3 & 20.4 & 22.1 & 21.4 \\
\hline $3-4$ & 1.2 & 1.8 & 1.5 & 12.7 & 10.5 & 11.4 \\
\hline$\geq 5$ & 1.2 & 0.5 & 0.8 & 8.0 & 4.5 & 5.9 \\
\hline
\end{tabular}

\section{Psychosociaal functioneren}

Gemiddeld scoren vrouwen wat minder goed op psychische gezondheid en sociaal functioneren dan mannen (Tabel 5). Tussen de leeftijdsgroepen blijken er nauwelijks verschillen te zijn in het psychisch functioneren, al scoort de jongste leeftijdsgroep (70-74 jaar) hier iets beter op dan de beide oudere leeftijdsgroepen. Bij het sociaal functioneren is er een duidelijkere trend: de scores verslechteren met het stijgen van de leeftijd. Over het algemeen wordt een beter psychisch en sociaal functioneren gerapporteerd als men samenwoont (respectievelijk $\mathrm{p}=0.000$ en $\mathrm{p}=0.001$ ) en naarmate het opleidingsniveau hoger is $(\mathrm{p}=0.000)$.

Een ander aspect van het sociaal functioneren, betreft het op bezoek gaan of bezoek ontvangen. Gemiddeld gaan de respondenten bijna twee keer per week op bezoek bij anderen, en ontvangen zij ruim twee keer per week bezoek van anderen. Bijna 15\% gaat echter geen enkele keer op bezoek bij anderen en $7 \%$ 
ontvangt geen bezoek van anderen. Vijf procent van de ouderen gaat niet op bezoek en ontvangt bovendien geen bezoek.

\begin{tabular}{|c|c|c|c|c|c|}
\hline \multirow{3}{*}{$\begin{array}{l}\text { Tabel } 5 \\
\text { Table } 5\end{array}$} & \multicolumn{5}{|c|}{$\begin{array}{l}\text { Gemiddelde scores (en standaarddeviaties) voor psychisch } \\
\text { en sociaal functioneren naar achtergrondkenmerken }\end{array}$} \\
\hline & \multicolumn{5}{|c|}{$\begin{array}{l}\text { Mean scores (and standard deviations) for mental health and } \\
\text { social functioning according to background characteristics }\end{array}$} \\
\hline & & $\begin{array}{l}\text { Psych } \\
\text { gezon }\end{array}$ & $\begin{array}{l}\text { sche } \\
\text { dheid }^{*}\end{array}$ & $\begin{array}{l}\text { Socią } \\
\text { functic }\end{array}$ & neren* \\
\hline \multirow[t]{2}{*}{ Geslacht } & man & 73.1 & (19.7) & 74.6 & $(28.4)$ \\
\hline & vrouw & 64.2 & $(21.9)$ & 69.4 & (29.8) \\
\hline \multirow[t]{3}{*}{ Leeftijd } & $70-74$ & 69.3 & $(21.4)$ & 75.4 & $(27.9)$ \\
\hline & $75-79$ & 66.9 & $(21.2)$ & 69.3 & $(29.6)$ \\
\hline & $80-84$ & 66.5 & $(21.9)$ & 66.6 & $(31.0)$ \\
\hline \multirow[t]{2}{*}{ Woonvorm } & alleenstaand & 63.8 & $(22.5)$ & 69.5 & $(29.1)$ \\
\hline & samenwonend & 70.5 & (20.3) & 72.9 & (29.4) \\
\hline \multirow{4}{*}{$\begin{array}{l}\text { Hoogst voltooide } \\
\text { opleiding }\end{array}$} & lagere school & 62.8 & $(22.0)$ & 66.7 & $(30.2)$ \\
\hline & Ibo & 67.7 & $(21.5)$ & 72.5 & (29.0) \\
\hline & $\mathrm{mbo}$ & 73.8 & $(18.8)$ & 75.4 & $(28.0)$ \\
\hline & hbo/universiteit & 76.4 & $(17.7)$ & 78.3 & (26.9) \\
\hline \multicolumn{2}{|l|}{ Totaal } & 67.9 & $(21.4)$ & 71.6 & (29.3) \\
\hline
\end{tabular}

* Range van 0-100 (score 100 geeft de meest gunstige score aan).

Van de totale populatie geeft $65 \%$ aan zich (bijna) nooit eenzaam te voelen ( $77 \%$ van de mannen en $55 \%$ van de vrouwen). Ongeveer een op de vijf vrouwen rapporteert dat zij zich in de afgelopen maand vaak of altijd eenzaam heeft gevoeld, tegenover ongeveer een op de tien mannen. Alleenstaanden voelen zich, zoals verwacht, vaker eenzaam dan samenwonenden. Ouderen die zich vaak eenzaam voelen scoren op psychische gezondheid en sociaal functioneren beduidend lager dan ouderen die zich (bijna) nooit eenzaam voelen $(\mathrm{p}=0.000)$.

\section{Zorggebruik}

In de 3 maanden voorafgaand aan de enquête heeft $69 \%$ van de respondenten contact gehad met de huisarts, $46 \%$ met een specialist en $8 \%$ is opgenomen geweest in het ziekenhuis (zie Tabel 6). Voor de respondenten die aangaven contact te hebben gehad met de huisarts of een specialist, was het aantal contacten respectievelijk $2.5 \pm 2.3$ en $2.2 \pm 1.9$. Per jaar is dat negen à tien keer. 
Het gemiddelde aantal contacten voor alle respondenten bedroeg respectievelijk $1.7 \pm 2.2$ en $1.0 \pm 1.7$. De aantallen verschilden niet voor mannen en vrouwen. Wel gingen naar verhouding iets meer mannen dan vrouwen naar de specialist. De ouderen die een beroep deden op de huisarts scoren slechter op zelfredzaamheid $(p=0.000)$ en psychisch en sociaal functioneren $(p=0.000)$, dan ouderen die de huisarts niet consulteerden.

\section{Tabel 6 Zorggebruik van respondenten (percentage gebruikers) \\ Table 6 Health care use of respondents (percentage users)}

\begin{tabular}{|c|c|c|c|c|c|c|}
\hline & & \multicolumn{3}{|c|}{ In de afgelopen 3 maanden } & \multicolumn{2}{|c|}{ Op moment van invullen } \\
\hline & & $\begin{array}{l}\text { contact } \\
\text { huisarts } \\
(\%)\end{array}$ & $\begin{array}{c}\text { contact } \\
\text { specialist } \\
(\%)\end{array}$ & $\begin{array}{c}\text { opname } \\
\text { ziekenhuis } \\
(\%)\end{array}$ & $\begin{array}{c}\text { thuiszorg } \\
(\%)\end{array}$ & $\begin{array}{c}\text { beschikking } \\
\text { mantelzorg } \\
(\%)\end{array}$ \\
\hline \multirow[t]{2}{*}{ Geslacht } & man & 67 & 49 & 8 & 18 & 92 \\
\hline & vrouw & 70 & 44 & 8 & 32 & 81 \\
\hline \multirow[t]{3}{*}{ Leeftijd } & $70-74$ & 66 & 45 & 7 & 17 & 88 \\
\hline & $75-79$ & 71 & 48 & 8 & 30 & 84 \\
\hline & $80-84$ & 72 & 44 & 10 & 43 & 82 \\
\hline \multirow[t]{2}{*}{ Woonvorm } & $\begin{array}{l}\text { alleen- } \\
\text { staand }\end{array}$ & 72 & 46 & 10 & 38 & 69 \\
\hline & $\begin{array}{l}\text { samen- } \\
\text { wonend }\end{array}$ & 67 & 46 & 7 & 19 & 95 \\
\hline \multirow{4}{*}{$\begin{array}{l}\text { Hoogst } \\
\text { voltooide } \\
\text { opleiding }\end{array}$} & $\begin{array}{l}\text { lagere } \\
\text { school }\end{array}$ & 71 & 47 & 9 & 33 & 82 \\
\hline & Ibo & 69 & 45 & 8 & 26 & 85 \\
\hline & mbo & 67 & 47 & 8 & 20 & 90 \\
\hline & $\begin{array}{l}\text { hbo/ } \\
\text { universiteit }\end{array}$ & 66 & 47 & 9 & 14 & 89 \\
\hline Totaal & & 69 & 46 & 8 & 26 & 85 \\
\hline
\end{tabular}

Op het moment van invullen van de vragenlijst maakte $26 \%$ van de respondenten gebruik van thuiszorg. Het overgrote deel hiervan $(90 \%)$ betreft huishoudelijke hulp. Hierbij bestaat een verschil tussen mannen en vrouwen: ongeveer een op drie vrouwen krijgt hulp, tegen een op vijf mannen. Bij ziekte zegt $85 \%$ van de respondenten terug te kunnen vallen op mantelzorg, veelal op de partner. Dit geldt vooral voor mannen. Vrouwen kunnen minder dan mannen (respectievelijk 61\% en 84\%) een beroep doen op partner of kind(eren), maar kunnen wel vaker dan mannen terugvallen op andere familieleden of buren (respectievelijk 19\% en 9\%).

Het beroep op de huisarts en het gebruik van thuiszorg neemt toe naarmate men ouder wordt. Het zorggebruik is over het algemeen ook groter, indien men 
alleenstaand is (behalve de contacten met de specialist). Alleenstaanden maken meer gebruik van formele zorg, maar zijn in het nadeel als het gaat om informele hulp. Slechts 22 respondenten $(0.6 \%)$ stonden op de wachtlijst voor een verpleeghuis en 168 ouderen (4.6\%) op een wachtlijst voor een verzorgingshuis.

\section{Gezondheidsbeleving}

Het gemiddelde rapportcijfer voor de eigen gezondheid bedraagt $6.6 \pm 1.7$ en dit verschilt niet tussen mannen en vrouwen. Over het algemeen schatten de ouderen hun gezondheid redelijk positief in: $31 \%$ geeft zich een 8 of hoger, terwij1 $24 \%$ zich een onvoldoende geeft. Naar verhouding zijn er iets meer ouderen in de hogere leeftijdscategorieën die zichzelf een onvoldoende geven. Het percentage dat de gezondheid als goed beoordeelt, is iets hoger onder mannen dan vrouwen en is hoger onder personen met een hogere opleiding. Er zijn nauwelijks verschillen gevonden in gezondheidsbeleving naar woonvorm.

Van de groep ouderen die zich een onvoldoende geeft voor zijn gezondheid, rapporteert bijna de helft dat de gezondheid achteruit is gegaan in vergelijking met 3 maanden geleden $\mathrm{Bij}$ ouderen met een goede gezondheidsbeleving (rapportcijfer 8-10) komt dit haast niet voor.

In Tabel 7 wordt de gezondheidsbeleving gerelateerd aan de drie domeinen die eerder besproken zijn (zelfredzaamheid, psychosociaal functioneren en zorggebruik). De gezondheidsbeleving is beter naarmate men gunstiger scoort op zelfredzaamheid $(p=0.000)$, psychisch en sociaal functioneren $(p=0.000)$, minder gebruik maakt van professionele zorg en meer beschikt over mantelzorg. De variabelen zelfredzaamheid, psychisch en sociaal functioneren, en zorggebruik (contact met huisarts), dragen allen significant bij aan de uitkomsten op gezondheidsbeleving. De invloed van zelfredzaamheid op gezondheidsbeleving is daarbij het grootst (40\% van de variatie wordt hieraan toegeschreven). De invloed van de overig genoemde variabelen is van minder groot belang (12\% van de verklaarde variatie). 
Tabel 7 Gezondheidsbeleving naar zelfredzaamheid, psychisch en sociaal functioneren (gemiddelde scores en standaarddeviaties), en zorggebruik (percentages)

Table 7 Health status according to functional status, mental health and social functioning (mean scores and standard deviations), and health care use (percentages)

\begin{tabular}{|c|c|c|c|c|}
\hline & & \multicolumn{3}{|c|}{ Rapportcijfer gezondheid } \\
\hline & & $\begin{array}{c}1-5 \\
\text { (slecht) }\end{array}$ & $\begin{array}{c}6-7 \\
\text { (redelijk) }\end{array}$ & $\begin{array}{l}8-10 \\
\text { (goed) }\end{array}$ \\
\hline \multirow[t]{2}{*}{ Zelfredzaamheid } & $\mathrm{ADL}^{*}$ & $20.3 \quad(6.7)$ & $14.4 \quad(4.2)$ & 11.9 \\
\hline & $\mathrm{HDL}^{\dagger}$ & $17.5 \quad(5.9)$ & 11.9 (4.9) & $8.4 \quad(3.0)$ \\
\hline \multicolumn{2}{|c|}{ Psychische gezondheid $^{\ddagger}$} & $51.7(21.4)$ & $68.2(18.6)$ & $80.2(16.4)$ \\
\hline \multicolumn{2}{|c|}{ Sociaal functioneren ${ }^{\ddagger}$} & $44.6(29.6)$ & $73.3(24.3)$ & $90.1(17.5)$ \\
\hline \multirow{3}{*}{$\begin{array}{l}\text { In de afgelopen } \\
3 \text { maanden }\end{array}$} & contact huisarts (\%) & 86 & 73 & 49 \\
\hline & contact specialist (\%) & 67 & 48 & 26 \\
\hline & ziekenhuisopname (\%) & 15 & 7 & 4 \\
\hline \multirow{2}{*}{$\begin{array}{l}\text { Op het moment } \\
\text { van invullen }\end{array}$} & thuiszorg (\%) & 49 & 27 & 8 \\
\hline & $\begin{array}{l}\text { beschikking } \\
\text { mantelzorg (\%) }\end{array}$ & 78 & 87 & 91 \\
\hline
\end{tabular}

* Range somscore ADL van 11-44 (score 11 geeft de minste beperking aan).

$\dagger$ Range somscore HDL van 7-28 (score 7 geeft de minste beperking aan).

$\ddagger$ Range van 0-100 (score 100 geeft de meest gunstige score aan).

Zestig procent van de ouderen met een slechte gezondheidsbeleving geeft aan dat men zelf weinig invloed kan uitoefenen op de gezondheidssituatie. Bij de groep ouderen met een goede gezondheidsbeleving is dit percentage slechts $10 \%$. Mannen rapporteren iets vaker dan vrouwen dat zij veel invloed kunnen uitoefenen op de eigen gezondheid (respectievelijk 43\% en 37\%). Naarmate men meer invloed denkt te kunnen uitoefenen op de gezondheid, scoort men gunstiger op zelfredzaamheid en psychosociaal functioneren, en gebruikt men minder professionele zorg. 


\section{Discussie}

\section{Zelfredzaamheid}

Wat betreft de ADL-activiteiten is men het vaakst hulpbehoevend bij voeten en teennagels verzorgen en het minst bij eten en drinken. Voor de HDL-activiteiten is men vooral vaak hulpbehoevend bij zwaar huishoudelijk werk. Zoals verwacht is een hoger percentage afhankelijk bij de uitvoering van HDL- dan bij ADL-activiteiten.

Voor een aantal activiteiten zijn vrouwen vaker afhankelijk van anderen dan mannen, zoals zichzelf wassen, trappen lopen, buitenshuis lopen, voeten en teennagels verzorgen, zwaar huishoudelijk werk en boodschappen doen. Mannen zijn vaker dan vrouwen afhankelijk bij het koken, kleren wassen en het bed verschonen. Uit eerder onderzoek onder ouderen blijkt ook dat vrouwen meer problemen hebben met zwaar huishoudelijk werk en boodschappen doen. Mannen hebben meer moeite met traditioneel meer door vrouwen uitgevoerde activiteiten als koken, kleren wassen en het bed verschonen. ${ }^{8}$

Er zijn aanwijzingen dat de ouderen in dit onderzoek het 'beter doen' wat betreft de ADL- en HDL-afhankelijkheid dan de ouderen uit vergelijkbaar onderzoek in Weert in 1988 en in Sittard in 1990. ${ }^{8}{ }^{12}$ Het percentage dat afhankelijk is, is over het algemeen nu lager voor de overeenkomstige leeftijdsgroepen (75-84 jarigen). In 1990 was bijvoorbeeld ongeveer 16\% van de vrouwen (80-84 jaar) afhankelijk van anderen bij zichzelf wassen tegen $6 \%$ in 2002, voor koken was dat $16 \%$ in 1990 tegen $8 \%$ in 2002, voor boodschappen doen $50 \%$ in 1990 tegen bijna $35 \%$ in 2002 en zwaar huishoudelijk werk $70 \%$ in 1990 tegen $50 \%$ in 2002 .

De zelfredzaamheid lijkt in de afgelopen 15 jaar verbeterd te zijn. Wel moet er rekening mee gehouden worden dat de formulering en antwoordcategorieën van de vragen iets verschilden in de verschillende studies. Elders is recentelijk echter eveneens aangegeven dat de zelfredzaamheid van thuiswonende 65plussers de afgelopen jaren is toegenomen. ${ }^{13}$ Ook het RIO spreekt de verwachting uit dat de vraag naar (voornamelijk ADL-) zorg zal veranderen, omdat de fysieke gezondheid van ouderen verbetert. ${ }^{14}$

\section{Psychosociaal functioneren}

Gemiddeld scoren vrouwen iets minder goed op psychisch functioneren dan mannen. Met het stijgen van de leeftijd verslechteren de scores, al zijn de verschillen in de scores tussen de leeftijdsgroepen gering. Deze bevindingen zijn een bevestiging van die in een Gronings onderzoek onder thuiswonende ouderen van 57 jaar en ouder. ${ }^{15}$ In een ander onderzoek waarin eveneens gebruik werd gemaakt van de SF-20, kwam naar voren dat psychisch functioneren in mindere mate afhankelijk is van de leeftijd. Metingen in de leeftijdsgroep vanaf 16 jaar lieten alleen voor de groep 75-plussers een slechtere score zien op psychisch functioneren. ${ }^{16}$ 
Evenals bij psychisch functioneren, zien we voor sociaal functioneren een slechtere score voor vrouwen in vergelijking met mannen, een slechtere score naarmate de leeftijd stijgt en naarmate men lager is opgeleid. Deze bevindingen zijn in overeenstemming met ander onderzoek. ${ }^{15}$

Wat betreft het sociaal functioneren is er een kleine groep ouderen die wellicht zorgen baart. $\mathrm{Zij}$ rapporteren niet bij anderen op bezoek te gaan en ook geen bezoek van anderen te ontvangen. Er zal nader onderzocht moeten worden of deze groep (5\% van de ouderen) ook op andere kenmerken slecht scoort. Wel is al gebleken dat de zelfredzaamheid en het psychosociaal functioneren onder de ouderen die niet op bezoek gaan of geen bezoek ontvangen, slechter is.

Psychosociaal functioneren geeft ook een samenhang te zien met eenzaamheidsgevoelens. Men scoort aanmerkelijk slechter op psychosociaal functioneren naarmate men zich meer eenzaam voelt. De gemiddelde score voor psychische gezondheid is bijna 70 (op een schaal van 0 tot 100) voor de gehele groep ouderen, terwijl de groep ouderen die zich vaak of altijd eenzaam voelt een score van rond de 40 heeft. Ook de zelfredzaamheidscores zijn slechter naarmate men zich meer eenzaam zegt te voelen.

\section{Zorggebruik}

Het blijkt dat een grote groep ouderen (69\%) contact heeft gehad met de huisarts in de 3 maanden voorafgaand aan het invullen van de vragenlijst. In 2002 lag dit percentage onder 65-plussers in Nederland op 85\%. ${ }^{1}$ Onder vrouwen, de 'hogere' leeftijdsgroepen, alleenstaanden en lager opgeleiden ligt het percentage dat een beroep deed op de huisarts, iets hoger.

Het gemiddelde aantal contacten met de huisarts ligt, omgerekend per jaar, op circa tien keer onder 'gebruikers'. Dit is laag in vergelijking met het landelijke aantal contacten onder 75-plussers. Dit aantal steeg in de periode 1997-2001 van 15 naar 19 keer per jaar. ${ }^{1}$

Iets meer mannen dan vrouwen hebben contact gehad met de specialist in de voorafgaande 3 maanden ( $49 \%$ tegen $44 \%$ ). Landelijk was het percentage in 2002 onder 65 -plussers $59 \%$, waarbij geen verschillen werden geconstateerd naar leeftijd, woonvorm of opleiding. ${ }^{1}$ Het aantal contacten ligt gemiddeld op negen keer per jaar onder gebruikers. Landelijk is dit aantal vijf keer (in 2002, onder 65-plussers). Bijna een op de 12 ouderen (8\%) was in de 3 voorafgaande maanden opgenomen geweest in het ziekenhuis, hetgeen iets hoger is dan landelijke cijfers over 65 -plussers. ${ }^{1}$

Ongeveer een op de vier onderzochte ouderen maakt gebruik van professionele thuiszorg, vooral huishoudelijke hulp. Evenals in ander onderzoek maken ouderen met een hogere leeftijd, alleenwonende en ouderen met een lagere opleiding vaker gebruik van thuiszorg. ${ }^{17}$ Uit verschillende publicaties van het Sociaal Cultureel Planbureau blijkt dat het opleidingsniveau van de hulpvrager een goede voorspeller is van de zorgvraag. ${ }^{18}$ Uit gegevens van het RIO blijkt dat naar verhouding meer vrouwen dan mannen gebruik maken van thuiszorg. ${ }^{14}$ Dit komt overeen met de gegevens uit dit onderzoek. Vrouwen 
leven gemiddeld langer dan mannen, maar brengen een zelfde aantal jaren in goede gezondheid door. Dit impliceert dat zij gemiddeld langer gezondheidsproblemen ondervinden en dan meer gebruik maken van zorg.

\section{Gezondheidsbeleving}

De ervaren gezondheid is een veelgebruikte samenvattende maat die verschillende gezondheidscomponenten integreert. De onderliggende gezondheidsaspecten variëren per persoon, maar hebben vaak betrekking op zowel de lichamelijke als de geestelijke gezondheid. ${ }^{19}$ Bij ouderen zijn voornamelijk chronische aandoeningen en lichamelijke beperkingen van belang bij de beoordeling van de eigen gezondheid. ${ }^{20-22}$ Uit deze gegevens blijkt ook dat zelfredzaamheid een belangrijke invloed uitoefent op gezondheidsbeleving.

Over het algemeen schatten de ouderen hun gezondheid positief in: 76\% geeft zich een rapportcijfer van zes of hoger. Het CBS heeft mensen gevraagd om aan te geven hoe gezond ze zich voelen, met als antwoordmogelijkheden: zeer goed, goed, gaat wel, soms goed en soms slecht, en slecht. De laatste drie antwoordcategorieën zijn geclassificeerd als minder goed ervaren gezondheid. In Nederland geeft $19 \%$ van de gehele populatie (alle leeftijden) aan dat men hun gezondheid als minder goed ervaart. De regio Zuid-Limburg scoort hierbij hoger dan het Nederlandse gemiddelde. ${ }^{23}$ Het hogere percentage dat zichzelf een onvoldoende geeft in deze studie in vergelijking met landelijke cijfers $(24 \%$ tegen 19\%), zal voor een deel komen door de hoge leeftijd in deze groep; naarmate men ouder wordt, neemt het percentage personen met een goede gezondheid af. Dit gegeven is in onze studie ook gevonden binnen de drie leeftijdscategorieën en is in overeenstemming met gegevens uit de literatuur. ${ }^{24}$

In dit onderzoek geven mannen zichzelf minder vaak een onvoldoende dan vrouwen. De verschillen in ervaren gezondheid tussen mannen en vrouwen zijn ook gevonden in ander onderzoek. ${ }^{24}$ Verschillen in samenlevingsvorm worden in de literatuur ook gevonden: samenwonenden rapporteren een betere gezondheidsbeleving. ${ }^{25}$ De verschillen in gezondheidsbeleving naar woonvorm zijn echter klein onder de onderzochte ouderen in dit onderzoek. In overeenstemming met eerder gevonden gegevens rapporteren in deze studie lager opgeleiden een slechtere ervaren gezondheid dan hoger opgeleiden. ${ }^{26}$

Gezondheidsbeleving is in dit onderzoek gerelateerd aan een aantal belangrijke domeinen. Ouderen met een slechte gezondheidsbeleving scoren, zoals verwacht, slechter op zelfredzaamheid en psychosociaal functioneren, rapporteren minder vaak op bezoek te gaan bij anderen, ontvangen minder bezoek en gebruiken meer professionele zorg.

Het grootste deel $(86 \%)$ van de ouderen met een slechte gezondheidsbeleving heeft een beroep gedaan op de huisarts in de 3 maanden voorafgaand aan het invullen van de vragenlijst. Wat de kenmerken zijn van de kleine groep van $14 \%$ die, ondanks een slechte gezondheidsbeleving, geen contact heeft gehad met de huisarts, zou nader onderzocht moeten worden. 


\section{Bruikbaarheid enquête}

De respons op de vragenlijst is met $76 \%$ goed te noemen. Vaak worden voor vergelijkbare postenquêtes onder thuiswonende ouderen percentages tussen de $45 \%$ en $65 \%$ gerapporteerd, al is in eerdere enquêtes in Limburgse steden eveneens een hoge respons behaald. ${ }^{89}$ Ongeveer 2 weken na het versturen van de vragenlijsten werd aan $44 \%$ van de populatie een herinnering gestuurd. Deze bestond uit een nieuwe vragenlijst plus begeleidende brieven, omdat gebleken is dat dit effectiever is om de respons te verbeteren dan alleen het versturen van een brief. $^{27}$

Het blijkt dat de ouderen, deels met hulp van anderen, de vragenlijst goed hebben kunnen invullen. Het aantal ontbrekende waarden was voor vrijwel alle vragen gering: voor de meeste variabelen tussen de $0 \%$ en $2 \%$ en voor een kleiner aantal tussen de 3\% en $4 \%$. De ouderen hebben in een enkel geval een beroep gedaan op de vrijwilligers van de ouderenbonden. De lijst is relatief kort, de gemiddelde duur om alles in te vullen bedroeg ongeveer 30 minuten. Dit kan de respons bevorderd hebben, hoewel een langere lijst het responspercentage niet altijd negatief beïnvloedt. ${ }^{28}$

Een postenquête lijkt een bruikbaar en kostenbesparend instrument om (relatief eenvoudige) gezondheidsgegevens over ouderen te verzamelen, of als selectie-instrument in onderzoek.

\section{Dankwoord}

De enquête waarover verslag wordt gedaan maakt deel uit van een onderzoek naar de effecten van huisbezoeken aan ouderen met ervaren gezondheidsproblemen, uitgevoerd door wijkziekenverzorgenden van de thuiszorg. Het onderzoek vindt plaats in samenwerking tussen de Universiteit Maastricht en de Thuiszorg Westelijke Mijnstreek te Sittard en wordt gefinancierd door Zorgonderzoek Nederland en Medische Wetenschappen (ZonMw).

Wij danken de Thuiszorg Westelijke Mijnstreek (Harry Heykens, Jan Houwen en Yvonne Monse), de Gemeente Sittard-Geleen-Born en de Ouderenbonden in de regio Sittard voor hun medewerking. Wij zijn Truus Custers, Carla Verheggen, Marijke Moll en Marion Gijbels erkentelijk voor hun ondersteuning bij de verzending van de enquêtes en de invoer van data. 


\section{Literatuur}

1. CBS [http://www.statline.cbs.nl]. Gezondheidskenmerken naar regio. Voorburg/Heerlen, 2000/2003.

2. GGD. Onderzoek Drenthe. 2002.

3. Deeg DJ, Knipscheer CP, Tilburg W, van. Autonomy and well-being in the aging population. Concepts and design of the Longitudinal Aging Study Amsterdam. Bunnik: Nederlands Instituut voor Gerontologie 1993.

4. Ormel J, Kempen GI, Wolffensperger EW, Steverink BJ, van Eijk LM, Brilman EI. NESTOR Program 'The Groningen Longitudinal Aging Study 1992-1996 on functional status and need for care'. Groningen: University of Groningen, Northern Research Centre for Health Issues, 1992.

5. Hofman A, Boerlage PA, Bots ML, et al. De prevalentie van chronische ziekten bij ouderen; het ERGO onderzoek. Nederlands Tijdschrift voor Geneeskunde 1995;139:1975-78.

6. Feskens EJM, Hoeymans FHGM, Tijhuis MAR, Viet L, Kromhout D. Zutphen Ouderen Studie; onderzoek naar leefstijl, chronische ziekten en kwaliteit van leven bij oudere mannen in Zutphen. Bilthoven: RIVM, 1996.

7. Nicolaides-Bouman A, van Rossum E, Kempen GI, Knipschild P. Effects of home visits by home nurses to elderly people with health problems: design of a randomised clinical trial in the Netherlands [ISRCTN92017183]. BMC Health Serv Res 2004;4(1):35.

8. Schrijnemaekers VJ, Haveman MJ. Evaluatie-onderzoek Poliklinische Geriatrie. Sittard: Maaslandziekenhuis en Rijksuniversiteit Limburg, 1990:1-38.

9. Kempen GI, Doeglas DM, Suurmeijer TP. Het meten van problemen met zelfredzaamheid op verzorgend en huishoudelijk gebied met de Groningen Activiteiten Restrictie Schaal (GARS) - een handleiding. Groningen: Noordelijk Centrum voor Gezondheidsvraagstukken, 1993.

10. Kempen GI, Brilman EI, Heyink JW, Ormel J. Het meten van de algemene gezondheidstoestand met de MOS Short-Form General health Survey (SF-20): een handleiding. Groningen: Noordelijk Centrum voor Gezondheidsvraagstukken, 1995.

11. van der Zee KI, Sanderman R. Het meten van de gezondheidstoestand met de RAND-36- een handleiding. Groningen: Rijksuniversiteit Groningen, Noordelijk Centrum voor Gezondheidsvraagstukken, 1993.

12. van Rossum E, Frederiks C, Knipschild P, Portengen J. Thuiswonende ouderen in de gemeenten Weert, Nederweert en Stramproy. Maastricht/Sittard, 1989:1-40.

13. Jacobs, Leyden V, Boer V. De leefsituatie van ouderen (55+) in Vlaanderen.: CBGSpublikaties, 2003.

14. Campen C, van, Gameren E, van. Vragen om hulp. Vraagmodel verpleging en verzorging. Den Haag: Sociaal en Cultureel planbureau, 2003.

15. Kempen GI, Brilman EI, Heyink JW, Ormel J. Het meten van de algemene gezondheidstoestand met de MOS Short-Form General health Survey (SF-20): een handleiding. Groningen: Noordelijk Centrum voor Gezondheidsvraagstukken, 1995.

16. Tijhuis MA, Picavet HS, Hoeymans N. Wat is de kwaliteit van leven van mensen in Nederland?: Volksgezondheid Toekomst Verkenning, Nationaal Kompas Volksgezondheid. Bilthoven, RIVM, 2001.

17. van der Meulen-Arts S, Francke A. Zijn er verschillen tussen bevolkingsgroepen in gebruik van thuiszorg?: Volksgezondheid Toekomst Verkenning, Nationaal Kompas Volksgezondheid. Bilthoven, RIVM, 2002.

18. Timmermans JM, Heide F, de Klerk M, Kooiker S, Ras M, van Dugteren FA. Vraagverkenning wonen en zorg voor ouderen. Rijswijk/Den Haag: Sociaal en Cultureel Planbureau/VUGA, 1997.

19. Tijhuis M, Hoeymans N. Wat is ervaren gezondheid en hoe wordt het gemeten?: Volksgezondheid Toekomst Verkenning, Nationaal Kompas Volksgezondheid. Bilthoven: RIVM, 2001.

20. Krause NM, Jay GM. What do global self-rated health items measure? Med Care 1994;32:930-942. 
21. Kempen GI, Brilman EI, Ormel J. Groningen longitudinal aging study. Een onderzoek naar het dagelijks functioneren, het welbevinden en de zorgbehoefte van ouderen. Tijdschrift voor Gerontologie en Geriatrie 1998;29(3):141-9.

22. Kempen GI, Miedema I, van den Bos GA, Ormel J. Relationship of domain-specific measures of health to perceived overal health among older subjects. J Clin Epidemiol 1998;51(1):11-18.

23. Giesbers H. Minder goede ervaren gezondheid 1995-1999.: Volksgezondheid Toekomst Verkenning, Nationaal Kompas Volksgezondheid. Bilthoven, RIVM, 2002.

24. Tijhuis M, Hoeymans N. Hoeveel mensen voelen zich niet gezond?: Volksgezondheid Toekomst Verkenning, Nationaal Kompas Volksgezondheid. Bilthoven, RIVM, 2002.

25. Tijhuis M, Hoeymans N. Ervaren gezondheid samengevat: Volksgezondheid Toekomst Verkenning, Nationaal Kompas Volksgezondheid. Bilthoven, RIVM, 2002.

26. Tijhuis M, Hoeymans N. Zijn er sociaal-demografische gezondheidsverschillen?: Volksgezondheid Toekomst Verkenning, Nationaal Kompas Volksgezondheid. Bilthoven, RIVM, 2002.

27. Hoffman SC, Burke AE, Helzlsouer KJ, Comstock GW. Controlled trial of the effect of length, incentives, and follow-up techniques on response to a mailed questionnaire. Am $\mathrm{J}$ Epidemiol 1998;148(10):1007-11.

28. Victor CR. Some methodological aspects of using postal questionnaires with the elderly. Arch Gerontol Geriatr 1988;7:163-172. 

Summary 
With the growth in the ageing population, home visiting programs by public health nurses have been developed aimed at improving the health and independent functioning of older persons and subsequently reducing hospital and nursing home admissions. Previous research in the Netherlands indicated that preventive home visits do not seem to be useful for the general population of older persons, but that these may be effective when restricted to those with poor health status. To test this assumption we carried out a new trial, focusing entirely on older persons in poor health. We also tested whether nurses with a less-intensive training (enrolled home nurses instead of public health nurses) are able to obtain convincing effects. The main aim of the study was to investigate the effects of systematic home visits by home nurses to older persons with poor self-reported health in terms of their health status, use of care services and costeffectiveness. A process evaluation of the content, compliance and experiences with the home visiting program was part of the study to assess the program's feasibility. A systematic literature review is included on the effectiveness of intensive home visiting programs targeting older persons with poor health status.

Chapter 1 presents background information on home visiting programs by nurses for older persons living in the community. Also, the rationale of a new clinical trial and its main objectives are presented.

In chapter 2 we describe the design of the trial. Eligibility of participants was determined through a postal questionnaire mailed in November 2002 to 4,901 persons (70-84 years) living at home in the south of the Netherlands (Sittard and surrounding areas). We excluded persons who reported their health status as moderate to good (self-rated health $\geq 6$, scale 1-10); who already received home nursing care on a regular basis; or who were on a waiting list for admission to a nursing home or home for older persons. There were sufficient eligible persons. Before randomization, 330 persons were stratified based on three prognostic factors: functional status, health change, and prior contact with a medical specialist. The participants in each of the strata were then randomized into an intervention $(\mathrm{n}=160)$ or a control group $(\mathrm{n}=170)$. Intervention participants received the home visiting program. The control group received usual care.

The intervention program consisted of eight visits over an 18-month period (approximately every 2 months a visit, always from the same nurse). The visits lasted between 60 and 90 minutes. In order to improve compliance, the nurses contacted the older persons by telephone 1-4 weeks after each visit. Three experienced home nurses conducted the visits under the supervision of a public health nurse. The nurses followed a structured protocol to assess health problems and risks, by interview. After the assessment, advice was given or the older persons were referred to professional and community services. The nurses were not part of a multidisciplinary team, but advice could be obtained from other disciplines within the home care organization, e.g. a dietician and an 
occupational therapist. A nurse geriatric specialist from the regional hospital could also be consulted, if necessary. The nurse specialist furthermore educated the nurses on important geriatric issues once every 6-8 weeks during the intervention period. The public health nurse and/or members of the research team supervised the nurses at weekly meetings. The home visits took place between February 2003 and October 2004.

As part of the process evaluation of the intervention, the nurses recorded the topics discussed at each visit, treated problems, advice given, referrals to other services, and compliance. After the intervention, the participants and nurses were asked about their experiences and expectations of the program.

The effects of the intervention program on primary health related outcomes were measured by means of postal questionnaires after 12, 18, and 24 months ( 6 months after the intervention). These outcomes are: self-rated health, functional status, quality of life and changes in self-reported problems. In addition, secondary health measures (e.g. health complaints, depressive complaints and mental health) were assessed after 18 months through individual interviews conducted by independent interviewers.

To assess the effects on health care use over the 24-month study period, we obtained data mostly from computerized databases of various medical administration offices. The services include: (inpatient) number of admissions and length of stay at the hospital, nursing home and home for older persons; (outpatient) number of contacts with medical specialists, general practitioners and paramedics; and hours of home care help. Associated costs were calculated and a cost-effectiveness analysis was conducted.

In chapter 3 the results of the process evaluation of the intervention program are presented. The home visiting program was largely carried out according to plan. Hundred and fifty-one of the 160 participants (95\%) received visits; 124 (78\%) were given the complete program of eight visits over an 18-month period and $27(17 \%)$ had, on average, four visits. The participants also received, on average, six phone calls. The average time spent on the visits in-home was 65 minutes. The most frequently discussed topics during the intervention were psychosocial functioning and functional status. On average, there were 10 treated problems and 11 interventions per participant over 18 months. Of all interventions, $38 \%$ were referrals to other services, $45 \%$ consisted of advice and $17 \%$ providing information. The overall compliance with the recommendations was $61 \%$. In general, the nurses and participants were satisfied with the program. A substantial proportion of the participants (66\%) thought they had greatly benefited, whereas the nurses expected the program to be beneficial for $48 \%$ of the participants. Based on the results of the process evaluation, we conclude that the home visits, performed by home nurses, were feasible and appreciated by both participants and nurses. 
Chapter 4 reports the results of the effect evaluation on health status. Data on primary outcome measures were available for 293 persons, 139 in the intervention (87\%) and 154 in the control group (91\%). At the end of the intervention period (18 months) only few differences could be detected between the study groups, e.g. the mean (adjusted) difference for self-rated health was -0.22 points $(95 \% \mathrm{CI}=-0.54-0.10$, scale $1-10)$, for activities of daily living (ADLs) -0.01 points $(95 \% \mathrm{CI}=-1.08-1.05$, scale $11-44)$, and for the SF-20 subscale on mental health -0.7 points $(95 \% \mathrm{CI}=-5.0-3.6$, scale $1-100)$. After 12 months, the intervention group had slightly better scores than the control group on self-rated health, functional status, and health change. None of the differences between the groups reached significance at the $5 \%$ level.

The secondary outcomes were available for 263 persons, 128 in the intervention $(80 \%)$ and 135 in the control group (79\%). For none of the measures, e.g. on health complaints, depressive complaints and mental status, statistically significant differences were found between the two groups.

Chapter 5 addresses the results of the effect evaluation on health care use and associated cost. The health care data had a high degree of completeness. The referrals made by the nurses caused an expected (small) increase in outpatient health care use, but no impact on the use of institutional health care was observed. With the exception of a higher distribution of aids and in-home modifications in favor of the intervention group, none of the results demonstrated statistically significant differences between the groups. The overall total cost per person for health care use, including cost for the home visiting program, was $€ 450$ higher in the intervention group than in the control group (overall differences not statistically significant). The home visiting program had a low chance $(10 \%)$ of being cost-effective.

Chapter 6 reports the results of a systematic review of randomized trials to assess the effects of intensive home visiting programs for older persons with poor health status. The intervention programs included - in line with our study at least four home visits per year and the home visiting period lasted 12 months or more. None of the included trials of sufficient methodological quality, that is seven out of eight (including our trial), had a significant favorable effect for the main analysis of the home visit group compared with the usual care group on mortality, health status, service use or cost.

In chapter 7 the main results and conclusions of our trial and the systematic review are presented. A number of methodological considerations are discussed, and we end with a section on implications for health care practice and further research. The main conclusion of this thesis is that home visiting programs appear not to be beneficial for older persons with poor health status within the health care setting of the Netherlands or comparable settings in Western countries. Because the positive results from a subgroup analysis from an earlier 
Dutch study could not be confirmed by the results of this larger replication study, we also conclude that post hoc subgroup analyses should be interpreted with caution.

There are no arguments to implement the home visiting program targeting older persons with poor health status to regular health care. Alternative strategies to improve the functional status and quality of life of communityliving older persons with poor health status should be developed and tested. Promising elements, such as care-coordination, self-management of chronic diseases, the integration of (medical) technology at older persons' homes together with in-person (nurse) contacts, the targeting of specific problems, e.g. related to mobility and other activities of daily living, deserve further attention in future research. 



\section{Samenvatting}

Summary in Dutch 
Het aantal ouderen is toegenomen en een verdere stijging wordt de komende jaren verwacht. Een consequentie hiervan is een grotere vraag naar professionele zorg met een daaraan gepaard gaande kostenstijging. In de afgelopen 25 jaar zijn er preventieve programma's ontwikkeld die als doel hebben om de gezondheid en het zelfstandig functioneren van ouderen te verbeteren en daarmee de (dure en schaarse) opnames in ziekenhuizen en verpleeghuizen te verminderen. Eén van deze programma's betreft preventieve huisbezoeken uitgevoerd door wijkverpleegkundigen. Eerder onderzoek in Nederland toonde aan dat dergelijke huisbezoeken niet zinvol zijn voor de algemene populatie ouderen, maar dat deze wellicht effectief zijn voor ouderen met ervaren gezondheidsproblemen. Om deze veronderstelling te toetsen, hebben we een nieuwe studie gedaan die gericht is op ouderen met een slechte gezondheidsbeleving. Tegelijkertijd keken we of wijkziekenverzorgenden (met een lager opleidingsniveau dan verpleegkundigen) in staat zijn om overtuigende effecten te behalen.

Het belangrijkste doel van deze studie is om de effecten na te gaan van systematische huisbezoeken door wijkziekenverzorgenden aan ouderen met gezondheidsproblemen op hun gezondheidstoestand, zorggebruik en hieraan gerelateerde kosten (kosteneffectiviteit). Een procesevaluatie van de inhoud, het opvolgen van aanbevelingen en de ervaringen met het huisbezoekenprogramma, was tevens onderdeel van de studie om de uitvoerbaarheid van het programma te beoordelen. Op het eind deden we ook nog een systematische literatuurstudie naar de effectiviteit van vergelijkbare huisbezoekenprogramma's voor ouderen met gezondheidsproblemen.

Hoofdstuk 1 presenteert de achtergrond van huisbezoekenprogramma's door verpleegkundigen aan zelfstandig wonende ouderen. Ook de beweegredenen van het nieuwe onderzoek en de belangrijkste doelstellingen komen aan bod.

In hoofdstuk 2 beschrijven we de opzet van het onderzoek, een gecontroleerd experiment. De geschiktheid van deelnemers werd bepaald met een korte schriftelijke vragenlijst. Deze werd in november 2002 aan 4901 zelfstandig wonende personen (70-84 jaar) in het zuiden van Nederland (Sittard en omgeving) toegezonden. Personen die hun gezondheid als redelijk tot goed ervaarden (gezondheidsbeleving $\geq 6$, op een schaal van 1 tot 10 ) werden uitgesloten van deelname. Ook personen die al regelmatig wijkverpleegkundige zorg ontvingen of op een wachtlijst stonden voor opname in een verzorgingshuis of verpleeghuis werden uitgesloten. Er waren voldoende geschikte deelnemers om aan het onderzoek mee te doen. Voordat randomisatie (loting) plaatsvond, werden de geselecteerde personen $(n=330)$ eerst gestratificeerd op drie prognostische factoren: functionele status, veranderingen in gezondheidstoestand en eerder contact met een medisch specialist (in de 3 maanden voorafgaand aan het invullen van de vragenlijst). De randomisatie vond plaats binnen elk stratum; 160 personen werden toegewezen aan de 
interventiegroep en 170 aan de controlegroep. De deelnemers in de controlegroep kregen de gebruikelijke zorg en de interventiegroep kreeg in aanvulling hierop het huisbezoekenprogramma.

Het interventieprogramma omvatte acht bezoeken over een periode van 18 maanden (ongeveer elke 2 maanden een bezoek, altijd van dezelfde wijkziekenverzorgende). De bezoeken duurden steeds 60 tot 90 minuten. Om het opvolgen van de adviezen te verbeteren hadden de wijkziekenverzorgenden 1-4 weken na elk bezoek telefonisch contact met de ouderen. Drie ervaren wijkziekenverzorgenden voerden de bezoeken uit onder supervisie van een wijkverpleegkundige. De wijkziekenverzorgenden volgden een gestructureerd protocol om gezondheidsproblemen en risico's op te sporen (door middel van een interview, geen lichamelijk onderzoek werd uitgevoerd). $\mathrm{Na}$ de inventarisatie werden adviezen verstrekt en/of werden de ouderen doorverwezen naar professionele hulpverleners of andere dienstverlenende instanties. De wijkziekenverzorgenden maakten geen deel uit van een multidisciplinair team, maar zij konden adviezen inwinnen bij andere disciplines binnen de thuiszorgorganisatie, bijvoorbeeld een diëtist en een ergotherapeut. Een geriatrisch verpleegkundig specialist verbonden aan een regionaal ziekenhuis konden zij ook raadplegen. Bovendien onderwees deze verpleegkundig specialist de wijkziekenverzorgenden over belangrijke geriatrische onderwerpen elke 6-8 weken gedurende de interventieperiode. De wijkverpleegkundige en/of leden van het onderzoeksteam begeleidden de wijkziekenverzorgenden tijdens wekelijkse besprekingen. De huisbezoeken vonden plaats tussen februari 2003 en oktober 2004. Als onderdeel van de procesevaluatie registreerden de wijkziekenverzorgenden de onderwerpen die besproken werden tijdens elk bezoek, de behandelde problemen, de gegeven adviezen, de verwijzingen naar andere hulpverleners, en de mate waarin de aanbevelingen werden opgevolgd.

De effecten van het interventieprogramma op primaire gezondheidsgerelateerde uitkomsten werden gemeten door middel van schriftelijke vragenlijsten na 12, 18 en ook nog na 24 maanden (6 maanden na afloop van de interventie). Deze uitkomsten zijn: gezondheidsbeleving, functionele status, kwaliteit van leven en veranderingen in zelfgerapporteerde problemen. Bovendien werden secundaire gezondheidsuitkomsten (bijvoorbeeld gezondheidsklachten, depressieve klachten en mentale gezondheid) na 18 maanden gemeten door middel van individuele interviews uitgevoerd door onafhankelijke interviewers.

Om de effecten na te gaan op het gebruik van zorgvoorzieningen over de gehele onderzoeksperiode ( 24 maanden), hebben we gegevens verkregen uit voornamelijk gecomputeriseerde gegevensbestanden van o.a. diverse zorgverzekeraars. Deze voorzieningen zijn: aantal opnames en opnamedagen in het ziekenhuis, verpleeghuis en verzorgingshuis; aantal contacten met medisch specialisten, huisartsen en paramedici; en uren thuiszorg. De daarmee verband houdende zorgkosten werden berekend en een kosteneffectiviteitanalyse werd uitgevoerd. 
In hoofdstuk 3 worden de resultaten gepresenteerd van de procesevaluatie van het huisbezoekenprogramma. Het huisbezoekenprogramma verliep grotendeels volgens plan. Honderd eenenvijftig van de 160 deelnemers (95\%) ontvingen bezoeken; $124(78 \%)$ kregen het complete programma van acht bezoeken over 18 maanden en de overige $27(17 \%)$ ontvingen gemiddeld vier bezoeken. De deelnemers hadden ook gemiddeld zes telefonische contacten met de wijkziekenverzorgende. De gemiddelde tijdsduur van de bezoeken aan huis bedroeg 65 minuten. De meest besproken onderwerpen tijdens de interventie waren psychosociaal functioneren en functionele status. Gemiddeld waren er 10 behandelde problemen en 11 interventies per deelnemer gedurende 18 maanden. Van alle interventies bestond $38 \%$ uit verwijzingen naar andere voorzieningen, $45 \%$ betrof advies en $17 \%$ het verschaffen van informatie. In totaal werden de aanbevelingen voor $61 \%$ opgevolgd. Over het algemeen waren de wijkziekenverzorgenden en de deelnemers tevreden over het programma. Veel deelnemers $(66 \%)$ dachten dat zij veel profijt hadden gehad van het programma, terwijl de wijkziekenverzorgenden profijt verwachtten voor $48 \%$ van de deelnemers. Gebaseerd op de resultaten van de procesevaluatie concluderen we dat de huisbezoeken, uitgevoerd door wijkziekenverzorgenden, uitvoerbaar zijn en gewaardeerd worden door zowel de deelnemers als de wijkziekenverzorgenden.

Hoofdstuk 4 rapporteert de effecten van de huisbezoeken op de gezondheid. Gegevens van de primaire uitkomstmaten waren beschikbaar voor 293 personen, 139 in de interventiegroep (87\%) en 154 in de controlegroep (91\%). Aan het einde van de interventieperiode (18 maanden) waren er slechts kleine verschillen waarneembaar tussen de twee groepen. Het gemiddelde verschil in gezondheidsbeleving was bijvoorbeeld -0.22 punten $(95 \% \mathrm{BI}=-0.54$ tot 0.10 , schaal 1-10), het gemiddelde verschil voor algemene dagelijkse levensverrichtingen (ADLs) was -0.01 punten $(95 \% \mathrm{BI}=-1.08$ tot 1.05 , schaal 11-44), en het gemiddelde verschil voor mentale gezondheid was -0.7 punten $(95 \% \mathrm{BI}=-5.0$ tot 3.6 , schaal $1-100)$. Na 12 maanden toonde de interventiegroep enigszins betere scores dan de controlegroep op ervaren gezondheid, functionele status en veranderingen in gezondheid. Geen van de verschillen tussen de groepen waren echter statistisch significant.

De secundaire uitkomsten waren beschikbaar voor 263 mensen, 128 in de interventiegroep (80\%) en 135 in de controlegroep (79\%). Voor geen enkele van de uitkomstmaten, bijvoorbeeld gezondheidsklachten, depressieve klachten en mentale gezondheid, werden statistisch significante verschillen gevonden tussen de interventie- en controlegroep.

Hoofdstuk 5 gaat over de effecten van de huisbezoeken op het gezondheidszorggebruik en de daarmee verband houdende kosten. De gegevens over het zorggebruik waren in hoge mate volledig. De door de wijkziekenverzorgende 
gemaakte verwijzingen veroorzaakten een verwachte, maar kleine toename van extramuraal zorggebruik (aantal huisartscontacten en uren thuiszorg), maar geen invloed was waarneembaar op het poliklinische en intramurale zorggebruik. Met uitzondering van een toename in de aanschaf van hulpmiddelen en het maken van aanpassingen in de woning in de interventiegroep, lieten geen van de resultaten een statistisch significant verschil zien tussen de twee groepen. De totale kosten per persoon van het zorggebruik, inclusief de kosten van het huisbezoekenprogramma, waren $€ 450$ hoger in de interventiegroep dan in de controlegroep (totaalverschillen niet statistisch significant). Het huisbezoekenprogramma had een lage kans $(10 \%)$ om kosteneffectief te zijn.

Hoofdstuk 6 rapporteert de resultaten van een systematische literatuurverkenning van gerandomiseerde studies om de effecten na te gaan van intensieve huisbezoekenprogramma's aan ouderen met een slechte gezondheidstoestand. De interventieprogramma's bestonden - zoals in onze studie - uit tenminste vier huisbezoeken per jaar en een periode van de huisbezoeken van 12 maanden of langer. In geen enkele van de ingesloten studies van voldoende methodologische kwaliteit, dat wil zeggen zeven van de acht studies (inclusief onze studie), hadden de bezoeken een significant gunstig effect op mortaliteit, gezondheid, zorggebruik of kosten.

In hoofdstuk 7 worden de belangrijkste resultaten en conclusies van het onderzoek gepresenteerd. Een aantal methodologische overwegingen wordt bediscussieerd, en we eindigen met de implicaties voor de praktijk en suggesties voor verder onderzoek. De belangrijkste conclusie van deze dissertatie is dat huisbezoekenprogramma's niet zinvol lijken voor oudere personen met (ervaren) gezondheidsproblemen in Nederland of in (westerse) landen met een vergelijkbaar gezondheidszorgsysteem. Omdat de positieve resultaten van een subgroepanalyse uit een eerder Nederlands onderzoek niet bevestigd konden worden door de resultaten van deze grotere replicatiestudie, concluderen we tevens dat post hoc subgroepanalyses met voorzichtigheid geïnterpreteerd dienen te worden.

Er zijn geen argumenten om het huisbezoekenprogramma voor ouderen met gezondheidsproblemen te implementeren in de reguliere gezondheidszorg. Alternatieve strategieën zullen ontwikkeld en getoetst dienen te worden om de functionele status en kwaliteit van leven van zelfstandig wonende ouderen met gezondheidsproblemen te verbeteren. Veelbelovende elementen, zoals zorgcoördinatie, zelfmanagement van chronische ziekten, de integratie van (medische) technologie in samenspraak met persoonlijk (verpleegkundig) contact, het zich richten op specifieke problemen, bijvoorbeeld gerelateerd aan mobiliteit en andere dagelijkse activiteiten, verdienen meer aandacht in toekomstig onderzoek. 



\section{Dankwoord}


Het is een geschenk dat ik dit proefschrift heb mogen afronden. Vele mensen hebben mij hierbij geholpen, het projectteam, ouderen in Sittard en omgeving, collega's, familie en vrienden. Ook diverse andere personen en organisaties hebben hun bijdrage geleverd. Ik ben iedereen dankbaar.

Pas in een later stadium heb ik mij gerealiseerd hoe bevoorrecht ik geweest ben om samen te mogen werken met mijn promotoren Paul Knipschild en Ruud Kempen, en copromotor Erik van Rossum. Paul, je was altijd even vriendelijk, natuurlijk tegendraads, vakkundig en ondersteunend. De koffie stond altijd klaar voor je tijdens onze wekelijkse overleggen. Ruud, je was altijd even snel en vakbekwaam met het becommentariëren van alle stukken. Ik ben blij dat je deel uitmaakte van het projectteam en later ook nog eens mijn promotor kon worden. Erik, ja die planningen waren me wat in het begin - ik ben er nooit echt gewend aan geraakt. Ik kan nu wel schrijven dat deze af en toe in de prullenbak terecht kwamen. Ik had eigenlijk maar één plan (dus toch) en dat was promoveren. Je was zonder meer een goede begeleider. Je enthousiasme, rust en vriendelijkheid en kennis van zaken waren waardevol. Ik dank jullie alle drie van harte voor de fijne begeleiding!

Ook wil ik Harry Crebolder, Jos Diederiks en Hans Fiolet danken als leden van de stuurgroep en medeaanvragers van dit project. De leden van de begeleidingscommissie, prof. Jan Hamers, prof. Gerjo Kok, prof. Marcel Olde Rikkert, prof. Martin Prins en prof. Andreas Stuck dank ik voor de positieve evaluatie van mijn proefschrift.

Truus Custers wil ik bedanken voor haar inzet als onderzoeksassistent. Van alle werkzaamheden rondom het versturen van bijna 5000 vragenlijsten en de invoer van de gegevens heeft Truus een groot deel voor haar rekening genomen. Ook Marion Gijbels, Marijke Moll en Carla Verheggen zijn behulpzaam geweest bij de werkzaamheden rondom het verspreiden van de vele vragenlijsten. Allen dank hiervoor. In het tweede jaar van het project is Annemarie Spaninks ons team komen versterken als onderzoeksassistent. Annemarie, veel dank voor je inzet en enthousiasme. Je stond altijd direct klaar om van alles en nog wat te regelen voor het project. Ook je samenwerking met de wijkziekenverzorgenden verliep prima en je zorgde dat alle gegevens netjes verzameld en ingevoerd werden. Je was ook 2 jaar mijn kamergenoot, dank voor alle fijne gesprekken die we hadden!

Ik was blij verrast met een voor mij destijds 'nieuwe wereld': de Thuiszorg. De samenwerking met de Thuiszorg Westelijke Mijnstreek (TWM, thans onderdeel van het Orbisch Medisch en Zorgconcern te Sittard) is uitstekend verlopen en ik kijk terug op een fijne tijd die ik met jullie allemaal heb mogen doorbrengen. Allereerst de wijkziekenverzorgenden Ria Claessens, Ine Janssen en Hanneke de Jongh - jullie enthousiasme en motivatie om het huisbezoekenprogramma uit te voeren was inspirerend. In de voorbereidingsfase, en ook later tijdens het programma, waren jullie bovendien leergierig en serieus om nieuwe dingen op te pakken. Door jullie inzet en harde werken is het programma bijna vlekkeloos 
verlopen. Veel dank! Wijkverpleegkundige, Yvonne Monse, dank voor de begeleiding van de wijkziekenverzorgenden tijdens o.a. de intervisiebijeenkomsten.

Alle medewerking en steun die we nodig hadden bij de thuiszorg is in eerste instantie mogelijk gemaakt door Harry Heykens, voormalig manager van TWM. Later trad Jan Houwen, zorgmanager, op als onze contactpersoon. Verder heeft Josée Kevers, PR en Communicatie, ons geholpen met een stukje mediabegeleiding om het project onder de aandacht van de ouderen te brengen. Gitte Pfeiffer zorgde ervoor dat we gegevens over het thuiszorggebruik van de ouderen konden raadplegen. Meer mensen van TWM hebben medewerking verleend aan ons project en ik wil iedereen hiervoor hartelijk bedanken.

Herbert Habets, die als geriatrisch verpleegkundig specialist verbonden is aan het Maaslandziekenhuis te Sittard (onderdeel Orbisch Medisch en Zorgconcern), zorgde voor de educatieve begeleiding van de wijkziekenverzorgenden tijdens het programma en adviseerde hen. Ook bij het opzetten van het bezoekprotocol van de huisbezoeken heeft hij een belangrijke rol gespeeld. Herbert, dank voor je bijdrage! Voor de instructie in het gebruik van de EasyCare vragenlijst zorgde Jack Hutten, destijds verbonden aan het Nivel, Utrecht. Met dank.

Veel ouderen in Sittard, Geleen en Munstergeleen hebben hun medewerking verleend aan het project. Ik was hier uiteraard erg blij mee en dank iedereen van harte. De interviewers Corry Claessens, Els Claessen, Lilian Lopez, Dymph van de Steene, Marijke Vaessen en Hilly Vroomen hebben de deelnemers aan het project thuis mogen opzoeken om gegevens te verzamelen. Dank voor jullie inzet om de interviews op een prettige en degelijke manier uit te voeren! Ook vele andere personen en organisaties hebben bijgedragen aan dit project. Ouderenbonden in de regio Sittard dank ik van harte voor het creëren van mogelijkheden om het project onder de aandacht te brengen bij de ouderen. De Gemeente Sittard-Geleen heeft ons project op diverse manieren gesteund. Samen met de vragenlijsten is er een begeleidende aanbevelingsbrief van wethouder Berry van Rijswijk uitgaan naar alle ouderen; de gemeente heeft de adresgegevens uit het bevolkingsregister verstrekt en tijdens de onderzoeksperiode ontvingen we wijzigingen in de persoonsgegevens van onze deelnemers. In het bijzonder wil ik Daphne Kagelmaker en Jack Ehlen hiervoor hartelijk bedanken!

Verder verleenden 19 huisartspraktijken (36 huisartsen) in Sittard, Geleen en Munstergeleen hun medewerking. Ook ontvingen wij vanuit de praktijken de gegevens over het zorggebruik van de deelnemers. Meer gegevens over het zorggebruik tijdens de onderzoeksperiode ontvingen we van het Maaslandziekenhuis, in het bijzonder dank ik Wilma Krämer; van de ziektekostenverzekeraars $\mathrm{CZ}$, in het bijzonder dank ik Daniëlle Baenen, VGZ, Ohra, Zilveren Kruis-Achmea, IZA, Avero-Achmea (voormalig AXA), DGVP en Univé; en van het $\mathrm{CZ}$ zorgkantoor ontvingen wij AWBZ data, in het bijzonder 
dank ik Brigitte Verjans. Van het CIZ (voormalig RIO) ontvingen we gegevens over alle geïndiceerde zorg, dank aan Marjolein Hendriks (deze data zijn echter niet opgenomen). Uiteraard ben ik iedereen dankbaar voor hun inzet en de fijne samenwerking.

Ton Ambergen, Silvia Evers, Herbert Habets en Patty Nelemans dank ik, als coauteurs van de opgenomen artikelen, van harte voor jullie inbreng en expertise. Voor het invoeren van de vele gegevens dank ik MEMIC, in het bijzonder Annemarie Mordant. Oud- (en voormalige) collega's van epidemiologie wil ik bedanken voor hun belangstelling en ook voor alle hulp en ondersteuning die ik van velen gekregen heb, met name John Aarts, Carolien Bastiaenen, Sandra Beijer, Brenda Bongaerts, Piet van den Brandt, Saskia Duijts, Christel van Gool, Yvonne Jaegers, Pieter Leffers, Marijke Moll, Harry van Montfort, Patty Nelemans, Martin Prins, Jos Slangen, Nathalie Slangen, Matty Weijenberg, Stefan de Vogel, Adri Voogd en Gwenn Wetzels. Ook oud-collega's van huisartsengeneeskunde wil ik bedanken voor hun belangstelling, in het bijzonder Ine Siegelaer, en verder van andere afdelingen, Hanny Prick, Jolanda van Haastregt, Rixt Zijlstra en Ton van Attekum. Van de collega's van het Nivel dank ik Wienke Boerma, Dionne Kringos en kamergenoot Lea Labaaij.

Hartverwarmend was al de steun en belangstelling die ik heb mogen ontvangen van al mijn vrienden, kennissen en vele andere personen in mijn omgeving. Te gek voor woorden. Ook mijn familie ben ik dankbaar voor hun geweldige steun, in het bijzonder mijn broers Piet, John, Rob en Ed, mijn vader en kinderen, Alex en Ilse Nicolaides, en Phedon. Mijn moeder mocht het helaas niet meer meemaken. Haar betrokkenheid en warme en stimulerende persoonlijkheid zal ik altijd missen.

Ans Bouman,

Amsterdam, juni 2008 


\section{About the author}

Ans Bouman was born in Amsterdam, the Netherlands, on 23 April 1956. She completed her secondary education (Atheneum-B) in 1974 at the 'Scholengemeenschap Noord', Amsterdam. She graduated a year later (1975) also from a high school in the United States of America. Directly after she started her study Movement Sciences at the Free University in Amsterdam and did her doctoral exam in 1982 with a major in exercise physiology and health. From 1983 until 1985 she worked as a researcher at the Physiological Laboratory of Leiden University on a project on individual differences in the human circadian system. She continued working as a researcher at the department of Environmental Preventive Medicine, Medical College of St Bartholomew's Hospital in London, Great-Britain (1986-1993). Among other projects, she participated in the 'Tar Reduction Study', a randomized trial of the effect of cigarette tar yield reduction on compensatory smoking. She also compiled two books on smoking statistics. Moving away from Great-Britain and living in Cyprus for 2 years, she moved back to the Netherlands in 1995. Her work as a researcher started again in 1999, when Ans became employed at the department of General Practice, Maastricht University, on the project 'Hartslag Limburg', a cardiovascular diseases prevention program. From there she moved to the department of Epidemiology of the same university, where she worked from 2002 until 2007 - resulting in the present thesis. From 2007 she works at NIVEL, the Netherlands Institute for Health Services, on the research project 'Mapping Professional Home Care in Europe'. 


\section{List of publications}

A Bouman, E v Rossum, P Nelemans, GIJM Kempen, P Knipschild. Effects of intensive home visiting programs for older persons with poor health status: a systematic review. BMC Health Serv Res, 2008;8:74.

A Bouman, E v Rossum, S Evers, T Ambergen, GIJM Kempen, P Knipschild. Effects on health care use and associated cost of a home visiting program for older people with poor health status: a randomized clinical trial in the Netherlands. J Gerontol A Biol Sci Med Sci 2008;63:291-297.

A Bouman, E v Rossum, T Ambergen, GIJM Kempen, P Knipschild. Effects of a home visiting program for older people with poor health status: a randomized clinical trial in the Netherlands. J Am Geriatr Soc, 2008;56(3):397-404.

A Nicolaides-Bouman, E v Rossum, GIJM Kempen, H Habets, P Knipschild. Home visiting program for older people with health problems: Process evaluation. J Adv Nurs, 2007;58(5):425-35.

A Nicolaides-Bouman, E v Rossum, GIJM Kempen, P Knipschild. Effects of home visits by home nurses to elderly people with health problems: design of a randomized clinical trial in the Netherlands [ISRCTN92017183]. BMC Health Serv Res, 2004;4(1):35.

AIE Nicolaides, E v Rossum, AM Spaninks, GIJM Kempen, H Heykens, J Houwen, Y Monse, PG Knipschild. De gezondheidstoestand van zelfstandig wonende ouderen in Sittard en Geleen. Ede, 7e Nationaal Gerontologiecongres, 2004;1 okt, 28.

A Nicolaides-Bouman, E v Rossum, P Knipschild, R Kempen, H Heykens, Y Monse. Effects of home visits by home nurses to elderly people with health problems. Amsterdam, 3rd European Nursing Congress, 5-8 October. Vulnerable groups in society: a nursing issue, 2003;117.

A Nicolaides-Bouman, E v Rossum, P Knipschild, R Kempen, H Heykens, Y Monse. Effects of home visits by home nurses to elderly people with health 
problems. Maastricht, The 4th European Doctoral Conference in Nursing Science, 2003;3-4 October, 36.

C Frost, FM Fullerton, AM Stephen, R Stone, A Nicolaides-Bouman, J Densem, A Semmence, N Wald. The Tar Reduction Study: randomized trial of the effect of cigarette tar yield reduction on compensatory smoking. Thorax, 1995;50:1038-1043.

A Nicolaides-Bouman, $\mathrm{N}$ Wald, $\mathrm{B}$ Forey, $\mathrm{P}$ Lee. International Smoking Statistics - a collection of historical data from twenty-two economically developed countries. Wolfson Institute of Preventive Medicine, London, and Oxford University Press, Oxford, 1993.

N Wald, AIE Nicolaides-Bouman. UK Smoking Statistics, 2nd edn. Wolfson Institute of Preventive Medicine, London, and Oxford University Press, Oxford, 1991.

NJ Wald, AIE Nicolaides-Bouman. Plasma retinal, beta-carotene and vitamin E levels in relation to the future risk of breast cancer. Brit J Canc, 1988;57:235

AIE Bouman, GA Kerkhof, PC Plooij-van Gorsel, M Lancel, DF Sumter, R vd Star. Sleep patterns for morning-type and evening-type individuals before and after a sleep-wake reversal. J Interd Cycle Res, 1985;16:122

AIE Bouman, GA Kerkhof, M Lancel, PC Plooij-van Gorsel, DF Sumter. Individual differences in the human circadian system. J Physiol, 1985;366:11

AIE Bouman, PC Plooij-van Gorsel, GA Kerkhof, M Lancel, DF Sumter. Sleep patterns of morning-type and evening-type individuals after a sleep-wake reversal. Psychophysiology, 1985;22:599

GA Kerkhof, AIE Bouman, PC Plooij-van Gorsel, M Lancel, DF Sumter. Differences between morning-type and evening-type individuals in the circadian rhythmicity of their performance. J Interd Cycle Res, 1985;16:137-8

PC Plooij-van Gorsel, AIE Bouman, GA Kerkhof, DF Sumter, M Lancel. Circadian variation of time estimation in morning-type and evening-type subjects. J Interd Cycle Res, 1985;16:150 